
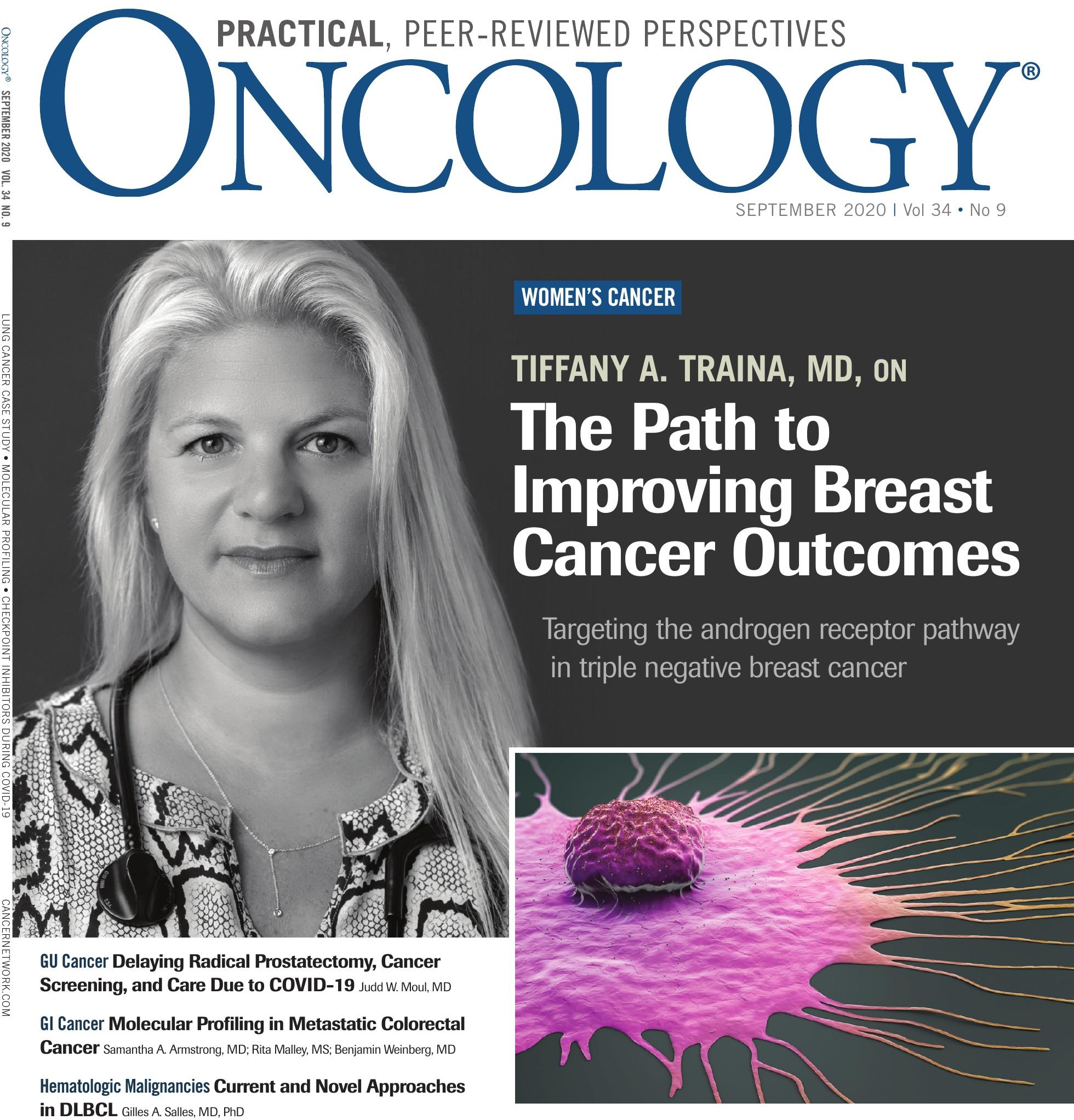

Immunotherapy Use of Immunotherapy in Patients With Cancer During the COVID-19 Pandemic

Andrey Soares, MD; Fabio A. Schutz, MD; Diogo Assed Bastos, MD; Denis Jardim, MD. PhD; Fernando Sabino Marques Monteiro, MD

Lung Cancer Recurrent EGFR-Mutated Non-Small Cell Lung Cancer Discovered by Abnormal Mammogram: Adjuvant/Frontline Metastatic Management Options Mehmet Sitki Copur, MD; Rudy Lackner, MD; Paul Rodriguez, MD; Adam J. Horn, MD; 


\section{WHAT MATTERS}

A diagnosis of prostate cancer was life-changing news for over 170,000 people per year
Cutting-edge treatments that are noninvasive so he can enjoy a life-changing trip around the world

\section{cancernetwork \\ home of the journal ONCOLOGY}

Oncology and CancerNetwork ${ }^{\text {m }}$ lead the industry with the latest insights from key opinion leaders in oncology through articles, peer perspectives, and interactive content that translates into clinical application for today's practicing oncologist.

Timely. Practical. Relevant. 


\section{IN THIS ISSUE}

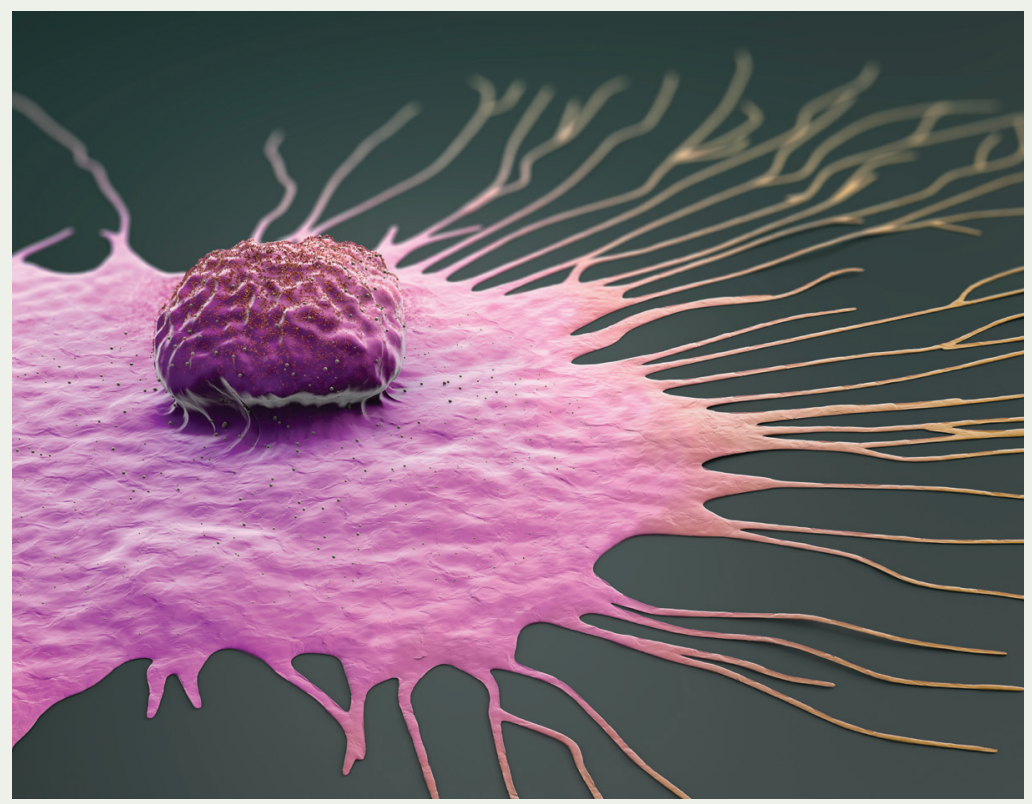

\section{WOMEN'S CANCER: Cover \\ The Path to Improving Breast Cancer Outcomes}

ONCOLOG $Y^{\circledR}$ recently sat down with Tiffany A. Traina, MD, a medical oncologist at Memorial Sloan Kettering (MSK) Cancer Center, to discuss recent developments in breast cancer care.

\section{Chairman's Letter \\ Choosing the Right Path}

Mike Hennessy Sr

343

\section{Letter to the Reader}

Delay in Cancer Screening and Diagnosis During the COVID-19 Pandemic: What Is the Cost?

Julie M. Vose, MD, MBA

344

GU Cancer: Clinical Update

Delaying Radical Prostatectomy, Cancer Screening, and Care Due to COVID-19

Judd W. Moul, MD

\section{cancernetwork.com}

Visit CancerNetwork.com, home of the journal ONCOLOGY ${ }^{B}$ and a web destination for oncologists seeking expert peer perspectives, podcasts, and other clinically practical features.

NEWS

Delayed Resection May Worsen Survival in Patients With Colorectal Cancer

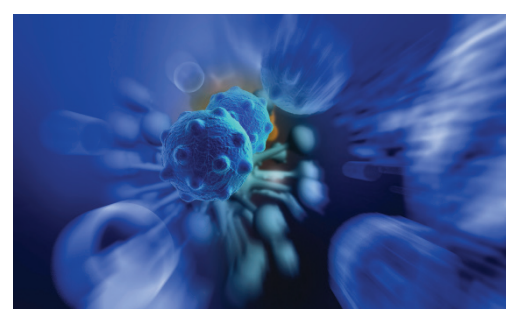

Cancernetwork.com/

delayed_resection_CRC

VIDEO

Robert L. Coleman, MD, on Looking Forward in Surgical Intervention for Ovarian Cancer

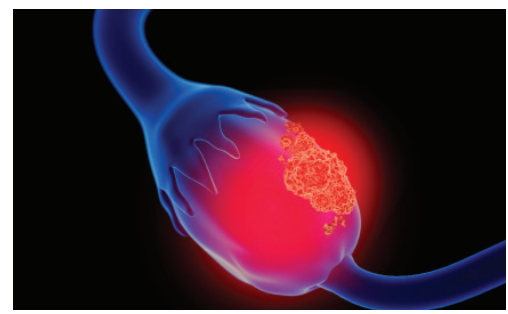

Cancernetwork.com/ ovariancancer_surgery

FDA APPROVAL

FDA Approves Azacitidine Tablets for Continued Treatment of Acute Myeloid Leukemia

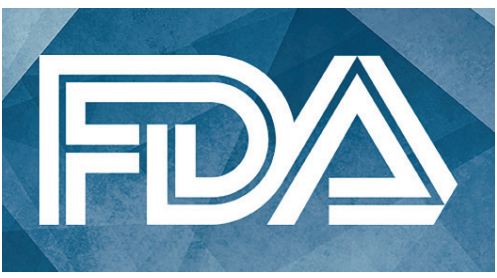

Cancernetwork.com/ azacitidine_aml 
IN THIS ISSUE

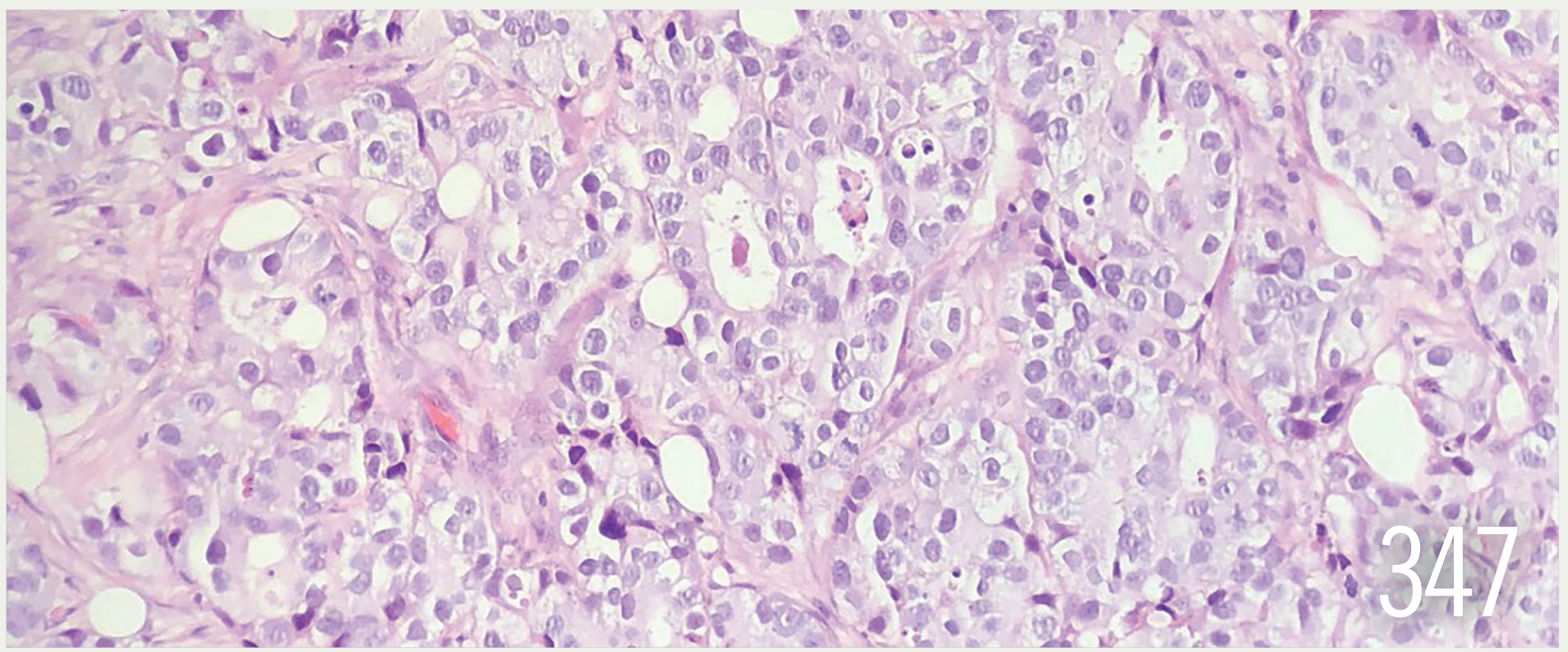

Lung Cancer: Case Study 347 Recurrent EGFRMutated Non-Small Cell Lung Cancer Discovered by Abnormal Mammogram: Adjuvant/ Frontline Metastatic Management Options

Mehmet Sitki Copur, MD; Rudy Lackner, MD; Paul Rodriguez, MD; Adam J. Horn, MD; Shellie Faris, MD; and Thomas Zusag, MD

\section{GI Cancer: Review 352 Molecular Profiling in Colorectal Cancer}

Samantha A. Armstrong, MD; Rita Malley, MS; and Benjamin A. Weinberg, MD

PERSPECTIVE: John L. Marshall, MD
PSYCHO ONCOLOGY: Review 367 Screening for Phase 1 Clinical Trials: An Opportunity to Evaluate Psychological States and Re-educate Patients on Prognosis?

Sarah Moreland, MD and Shiraj Sen, MD

IMMUNOTHERAPY: Clinical Quandaries

370 Use of

Immunotherapy in

Patients With Cancer

During the COVID-19

Pandemic

Andrey Soares, MD; Fabio A. Schutz, MD; Diogo Assed Bastos, MD; Denis Jardim, MD, PhD; and Fernando Sabino Marques Monteiro, MD
TELEHEALTH: Medical

Economics

377 Is Telehealth Here to Stay?

\section{HEMATOLOGICAL}

MALIGNANCIES: Continuing

Education

379 Current and Novel

Approaches in DLBCL

Gilles A Salles, MD, PhD
Published in affiliation with

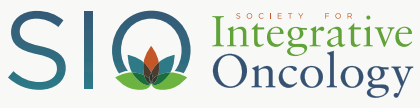

JPER $R^{\oplus}$ THE EDITORS ARE PLEASED to ANNOUNCE the availability of our new parent company's continuing education activities. gotoper.com We've picked this one especially for our ONCOLOGY ${ }^{\circledR}$ readers. Go to: https://bit.ly/2IRAknZ

Advertiser and advertising agency recognize and accept that the following language appears within the publication: "All statements, including product claims, are those of the person or organization making the statement or claim. The publisher does not adopt any such statement or claim as its own, and any such statement or claim does not necessarily reflect the opinion of the publisher."

Advertiser and advertising agency accept and assume liability for all content (including text representations, illustrations, opinions and facts) of advetisements printed, and also assume responsibility for any claims made against the publisher arising from or related to such advertisements. In the event that legal action or a claim is made against the publisher arising from or related to such advertisements, advertiser and advertising agency agree to fully defend, indemnify and hold harmless the publisher, and to pay anyjudgment, expenses and legal fees incurred by the publisher as a result of said legal action or claim. The publisher reserves the right to reject any advertising which he feels is not in keeping with the publication's standards.

Publisher is not liable for delays in delivery and/or non-delivery in the event of an act of God, action by any government or quasi-governmental entity, fire, flood, insurrection, riot, explosion, embargo, strikes (whether legal or illegal), labor or material shortage, transportation interruption of any kind, work slow-down, or any condition beyond the control of publisher affecting production or delivery in any manner.
ONCOLOGY (ISSN 0890-9091) is published monthly by MultiMedia Healthcare LLC, 2 Clarke Drive, Suite 100 Cranbury, N 08512. Annual subscription rates: US, $\$ 199$ and Canada, $\$ 219$; students and nurses, $\$ 96$; international, \$249. Single copies: $\$ 20$ each. Institutional US, \$299; Canada, \$329; international, \$375. Periodicals postage paid at Trenton, $\mathrm{NJ}$ and at additional mailing offices. POSTMASTER: Please send address changes

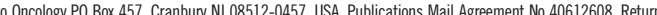
Undeliverable Canadian Addresses to: IMEX Global Solutions, PO Box 25542 London ON N6C 6B2. Canadian G.S.T number: R-124213133RT001. Printed in U.S.A.

For address changes, please notify the Circulation Department by visiting www.surveymonkey.com/s/subscriptions, or by mail to ONCOLOGY ( \) 2020 MJH Life Sciences, PO Box 457, Cranbury N 08512-0457. Send old address, new address and attach a copy of mail label, if possible. 


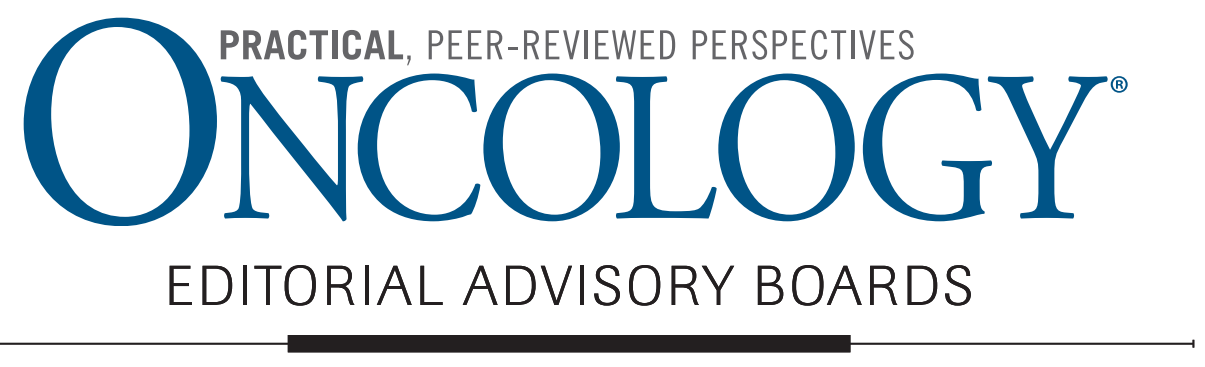

MISSION

STATEMENT

ONCOLOGY $Y^{\circledR}$ and its website, CancerNetwork.com, provide oncologists with the practical, timely, clinical information they need to deliver the highest level of care to their patients. Expert authors and peer review ensure the quality of ONCOLOGY ${ }^{\circledR}$ and CancerNetwork.com's articles and features. Focused discussions capture key clinical take-aways for application in today's time-constrained practice environment.

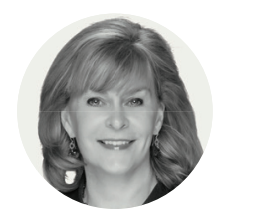

Julie M. Vose, MD, MBA Omaha, NE

\section{EDITORS-IN-CHIEF}

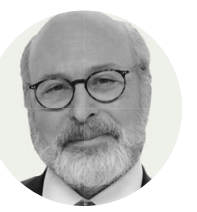

Howard S. Hochster, MD New Brunswick, NJ

\section{EDITORIAL BOARD}

\section{TUMOR CHAIRS}

BREAST CANCER

Sara A. Hurvitz, MD, Los Angeles, CA

GENITOURINARY CANCER

Robert A. Figlin, MD, Los Angeles, CA

\section{BOARD MEMBERS}

BREAST CANCER

William J. Gradishar, MD, FACP, Chicago, IL

Tari King, MD, Boston, MA

Vered Stearns, MD, Baltimore, MD

Melinda L. Telli, MD, Palo Alto, CA

CANCER SURVIVORSHIP

Matthew J. Matasar, MD, MS, New York, NY

COLORECTAL/GASTROINTESTINAL CANCER

Edward Chu, MD, Pittsburgh, PA

Mehmet Sitki Copur, MD, FACP, Omaha, NE

Daniel Haller, MD, Philadelphia, PA

John L. Marshall, MD, Washington, DC

Shubham Pant, MD, Houston, TX

Matthew B. Yurgelun, MD, Boston, MA

Mehmet Sitki Copur, MD, FACP, Omaha, NE

GENITOURINARY CANCER

L. Michael Glodé, MD, FACP, Denver, CO

Paul Mathew, MD, Boston, MA

Elisabeth Heath, MD, FACP, Detroit, MI

William Oh, MD, New York, NY

GYNECOLOGIC ONCOLOGY

Mario M. Leitao Jr, MD, New York, NY

Franco Muggia, MD, New York, NY
GASTROINTESTINAL CANCER

Tanios S. Bekaii-Saab, MD, Phoenix, AZ

HEAD AND NECK CANCER

Eric J. Sherman, MD, New York, NY

HEAD AND NECK CANCER

Apar K. Ganti, MD, MS, FACP, Omaha, NE

HEALTH ECONOMICS

Nora Janjan, MD, Dallas, TX

HEMATOLOGIC MALIGNANCIES

Danielle M. Brander, MD, Durham, NC

Christopher R. Flowers, MD, Houston, TX

Steven T. Rosen, MD, Duarte, CA

Naval G. Daver, MD, Houston, TX

Ehab L. Atallah, MD, Milwaukee, WI

INFECTIOUS DISEASE

Genovefa Papanicolaou, MD, New York, NY

INTEGRATIVE ONCOLOGY

Ting Bao, MD, New York, NY

Linda Carlson, PhD, RPsych, Calgary, Alberta,

Canada

LUNG CANCER

David S. Ettinger, MD, Baltimore, MD

James L. Mulshine, MD, Chicago, IL

Edward S. Kim, MD, Charlotte, NC

Jennifer W. Carlisle, MD, Atlanta, GA

MELANOMA

Richard D. Carvajal, MD, New York, NY

Jason Luke, MD, FACP, Pittsburgh, PA
HEMATOLOGIC MALIGNANCIES

C. Ola Landgren, MD, PhD, New York, NY

LUNG CANCER

Joshua M. BaumI, MD, Philadelphia, PA

NEURO-ONCOLOGY

David A. Reardon, MD, Boston, MA

Stuart A. Grossman, MD, Baltimore, MD

Nicole A. Shonka, MD, Omaha, NE

PEDIATRIC ONCOLOGY

David G. Poplack, MD, Houston, TX

Richard A. Drachtman, MD, New Brunswick, NJ

PROSTATE CANCER

Tomasz M. Beer, MD, Portland, OR

E. David Crawford, MD, Denver, CO

Judd W. Moul, MD, FACS, Durham, NC

PSYCHO-ONCOLOGY

Daniel C. McFarland, DO, New York, NY

Michelle Riba, MD, Ann Arbor, MI

RADIATION ONCOLOGY

Louis Potters, MD, FACR, Hempstead, NY James B. Yu, MD, MHS, New Haven, CT

SARCOMA

Kenneth Cardona, MD, FACS, Atlanta, GA

SUPPORTIVE AND PALLIATIVE CARE

Thomas J. Smith, MD, FACP, Baltimore, MD

N. Simon Tchekmedyian, MD, Long Beach, CA

SURGICAL ONCOLOGY

Burton L. Eisenberg, MD, Newport Beach, CA

\section{INTERESTED IN SUBMITTING TO ONCOLOGY ${ }^{\circledR}$ ?}

Please contact senior editor Kevin Wright at KWright@mjhlifesciences.com for submission guidelines or more information. 


\section{Choosing the Right Path}

Triple-negative breast cancer (TNBC) has become recognized as a heterogeneous, hormone-driven disease, and increasing evidence suggests that within a subset of these tumors, a role for androgen signaling exists. In this issue of ONCOLOGY ${ }^{\circledR}$, we speak with Tiffany A. Traina, MD, a medical oncologist with Memorial Sloan Kettering Cancer Center, about her work in targeting the androgen receptor (AR) pathway in patients with TNBC.

"I think we're still trying to understand the best biomarker to be able to predict for benefit. It may be something beyond just the presence of the receptor, but perhaps more of a composite marker to predict benefit," says Traina of the recent work with AR signaling. "Some data show that the AR may be relevant not just in TNBC, but also in estrogen receptor (ER)-positive breast cancer, where the coexpression of ER and AR is quite high."

Traina also discusses the expanding use of immunotherapies for TNBC. 'We've come to see that for metastatic disease, introducing these agents early in the first-line setting shows greater promise than using them in a later line," notes Traina. "We are also now seeing, from early-stage trials, that moving these checkpoint inhibitors up into the neoadjuvant setting, even potentially the adjuvant setting, has great benefit."

For this month's clinical quandary, you will read about the challenges of starting immunotherapy for patients with cancer during the coronavirus disease 2019 pandemic. How have treatment protocols changed? What can we do to ensure the best possible treatment with the lowest possible risk? Read on to find out.

Also in this issue, you will read a review of molecular profiling in metastatic colorectal cancer (mCRC). The implementation of next-generation sequencing has changed the landscape of treatment in $\mathrm{MCRC}$, identifying mutational targets and improving outcomes. In an accompanying editorial commentary, John L. Marshall, MD, director of the Ruesch Center for the Cure of Gastrointestinal Cancers, elaborates on the potential for molecular profiling to transform cancer care around the world. He states that cancer care can "no longer be a luxury item, only for the rich."

Within these pages, you will also read a review of screening for phase 1 clinical trials; a commentary from Judd W. Moul, MD, of the Duke Cancer Institute on the effect of delayed radical prostatectomy in patients with prostate cancer; and the case study of a 68-year-old woman with the rare occurrence of breast metastases from EGFR-mutated non-small cell lung cancer.

I hope you find our journal helpful in caring for your patients through what is likely one of the most challenging times in their lives. As always, thank you for reading.

Mike Hennessy Sr

Chairman and Founder of ONCOLOGY' parent company, MJH LIfe Sciences ${ }^{m}$

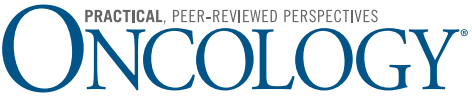

SEPTEMBER 2020 • VOL. 34 • NO. 9

\section{EDITORIAL}

KRISTIE L. KAHL Editorial Director

KEVIN WRIGHT Senior Editor

HANNAH SLATER Assistant Editor

MATTHEW FOWLER Assistant Editor

JENNIFER POTASH Copy Chief

RACHELLE LALIBERTE, PAUL SILVERMAN Copy Supervisors

CHENEY BALTZ, GEORGINA CARSON, KIRSTY MACKAY Copy Editors

\section{DESIGN \& PRODUCTION \\ ROBERT MCGARR Creative Director KRISTEN MORABITO Art Director \\ RACHEL KEATLEY Graphic Designer \\ JONATHAN SEVERN Circulation Director \\ KEYONNA GRAHAM Production Director}

\section{Publishing \& SALES}

BRIAN HAUG Executive Vice Presiden 609-325-4780•bhaug@mmhgroup.com

MARC MATHEWS Vice President Oncology 609-819-5245 • mmathews@mjhlifesciences.com

MICHELLE JANIN Director of Sales

732-429-4316•mjanin@mmhgroup.com

PATRIC PASTORE National Accounts Associate 609-955-1694 • ppastore@mjhlifesciences.com

\section{AUDIENCE DEVELOPMENT}

KELLY KEMPER Audience Development Manager

\section{CORPORATE}

MIKE HENNESSY SR Chairman \& Founder

JACK LEPPING Vice Chairman

MIKE HENNESSY JR President \& CEO

NEIL GLASSER, CPA/CFE Chief Financial Officer

TOM TOLVÉ Executive Vice President, Operations

JOE PETROZIELLO Executive Vice President, Global Medical Affairs and Corporate Development

SILAS INMAN Senior Vice President, Content

JOHN MORICONE Senior Vice President, I.T. \& Enterprise Systems

JoY PUZZO Senior Vice President, Audience Generation \& Product Fulfillment

SHARI LUNDENBERG Vice President, Human Resources \& Administration

CHRIS HENNESSY Vice President, Mergers \& Acquisitions JEFF BROWN Executive Creative Director, Creative Services

\section{SUBSCRIPTIONS}

888-527-7008

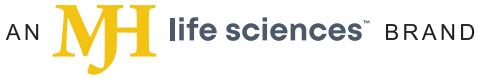




\title{
Delay in Cancer Screening and Diagnosis During the COVID-19 Pandemic: What Is the Cost?
}

\author{
Julie M. Vose, MD, MBA \\ Chief, Hematology/Oncology \\ Neuman M. and Mildred E. Harris Professor \\ University of Nebraska Medical Center \\ Omaha, NE
}

D uring the height of the coronavirus disease 2019 (COVID-19) pandemic, many health care facilities needed to focus on screening for and treating patients with known or suspected COVID-19. This resulted in the diversion of health care workers and resources. As a result, standard cancer screening such as breast cancer screenings dropped by $89.2 \%$ and colorectal cancer screenings dropped by $84.5 \%$ through May 2020. ${ }^{1}$ These pandemic control efforts translated into a significant decline in the number of new cancer diagnoses, resulting in a decrease of $65.2 \%$ incidence of new cancer diagnoses in April 2020. ${ }^{1}$ In evaluating specific types of cancer diagnosis, patients with a new diagnosis of melanoma dropped $67.1 \%$ in April 2020 compared with 2019 and a diagnosis of a new lung cancer which dropped $46.8 \%$ over the same time. ${ }^{1}$ This study and others have demonstrated an alarming decrease in the diagnosis of new cancers which will potentially increase the number of patients with later-stage cancers leading to decreased survival for these patients. ${ }^{2,3}$ Using National Health Service (NHS) data on cancer diagnosis and hospital administrative datasets, the investigators' modeling study evaluated estimated changes in future death rates. Across different scenarios as compared with prepan- demic figures, the investigators estimated a $7.9 \%$ to $9.5 \%$ increase in deaths from breast cancer up to 5 years from diagnosis. ${ }^{3}$ In addition, a $15.5 \%$ to $16.6 \%$ increase in colorectal cancer deaths and a $4.8 \%$ to 5.3 $\%$ increase in lung cancer deaths were estimated. ${ }^{3}$ In addition to health care facilities decreasing routine screening and nonurgent surgeries to increase capacity for patients with COVID-19 complications, patients themselves have in some cases expressed concern about visiting the health care facilities to do routine cancer screenings for fear of COVID-19 exposure. However with current strict measures in place at all health care facilities, this would be a very low risk procedure for the patient.

Prior to the COVID-19 pandemic, the US cancer statistics had continued to improve over the last few decades including a $25 \%$ drop in cancer mortality over the past 25 years. ${ }^{4}$ However, with less cancer screening comes the potential for malignancies to be diagnosed at a later stage. This could translate into worse outcomes when patients are diagnosed later in the course of the disease making treatment more difficult and the cancer less able to be cured. We do need to encourage patients to continue their standard cancer screenings during these difficult times, so that the progress in cancer diagnosis, treatment, and survival continues to improve over the next decades to come. As our nation slowly and safely opens up again, cancer screening and diagnosis needs to continue to play an important part in our standard healthcare measures. Without going back to pre-COVID screening numbers, our cancer diagnosis and mortality rates could revert to numbers seen many years ago without the benefit of our current technological advancements. Those of us in the oncology community should join forces with the primary care physicians and health care systems to enhance opportunities for cancer screening to reverse this concerning trend.

\section{REFERENCES}

1. London JW, Fazio-Eynullayeva E, Palchuk $\mathrm{MB}$, et al: Effects of the COVID-19 pandemic on cancer-related patient encounters. JCO Clinical Cancer Inform. 2020;4: 657-665. doi: 10.1200/CCI.20.0006

2. Epic Health Research Network. Preventive cancer screenings during COVID-19 pandemic. May 1, 2020. Accessed August 20, 2020. https://www.ehrn.org/wp-content/ uploads/Preventive-Cancer-Screeningsduring-COVID-19-Pandemic.pdf

3. Maringe C, Spicer J, Morris M, et al. The impact of the COVID-19 pandemic on cancer deaths due to delays in diagnosis in England, UK: a national, population-based, modelling study. Lancet Oncol. 2020;21(8):1023-1034. doi:10.1016/S1470-2045(20)30388-0

4. Siegel RL, Miller KD, Jemal A. Cancer statistics, 2019. CA Cancer J Clin. 2019;69(1):7-34. doi:10.3322/caac.21551 


\section{Delaying Radical Prostatectomy, Cancer Screening, and Care Due to COVID-19}

\section{Two Retrospective Analyses Show No Associated Adverse Outcomes With Delayed RP}

Kevin Wright

The results of 2 studies showed no association between delayed radical prostatectomy (RP) and adverse oncological outcomes, supporting current recommendations of urologic societies for surgical treatment of patients with intermediate- and high-risk prostate cancer during the coronavirus disease 2019 (COVID-19) pandemic.

A retrospective analysis published in the Journal of Urology indicated that a delay of up to 12 months did not result in worse outcomes when compared with immediate surgery within 3 months of diagnosis. ${ }^{1}$

When compared with patients receiving RP within 3 months of diagnosis, patients undergoing surgery 4 to 12 months after diagnosis did not have increased odds of adverse pathology, upgrading on RP, or node-positive disease.

The investigators accrued data from the National Cancer Database for men with intermediate-and high-risk prostate cancer who received RP between 2010 and 2016. Surgery $\leq 3$ months from diagnosis was defined as immediate RP, with delayed RP being evaluated in 3-month intervals up to 12 months (4-6 months, 7-9 months, 10-12 months).

Overall, the analysis included 128,062 patients with a median age of 63 years (interquartile range [IQR], 58-67) and a median PSA at diagnosis of $6.3 \mathrm{ng} / \mathrm{mL}$. Just over

\section{MEET OUR EXPERT}

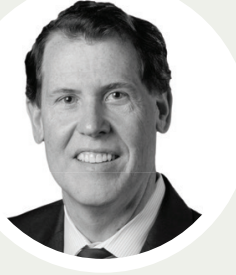

Moul is a urologic oncologist at the Duke Cancer Institute in Durham, NC.

\section{Are COVID-19 Delays a Basis for Concern? Can We Use This for Future Good? Judd W. Moul, MD}

In my 30-plus years in medicine and as a urologic oncologist, I have never had to triage civilian patients for elective surgery, but that is exactly what we were asked to do from March 2020 onward as the coronavirus disease 2019 (COVID-19) pandemic engulfed our communities. We had the opportunity to report our early experience with triage for localized prostate cancer in the May 2020 issue of ONCOLOGY ${ }^{\circledR}{ }^{1}$ From mid-March to midMay 2020, I postponed about 20 patients with low- to high-risk localized prostate cancer scheduled for radical prostatectomy (RP). However, as I write now in mid-August, I can report that we cared for these men with a surgical delay averaging less than 3 months. We were fortunate that our health system had sufficient personal protective equipment and that the impact of COVID-19 in our area was less severe than in many other parts of the United States, which allowed our teams to ramp back up to full capacity by early June 2020. Recent articles by Diamand et al in the World Journal of Urology and Ginsberg et al in the Journal of Urology found that modest delay in RP did not impact outcomes. ${ }^{2,3}$ I am not surprised. For all but the most severe cases of localized prostate cancer, modest delay of 2 to 6 months is unlikely to affect long-term survival.

However, this does not mean that COVID-19 will not affect the survival and outcomes of 


\section{Retrospective Analyses Continued from page 344}

half $(50.1 \%)$ of the patients had Grade Group (GG) 2 disease. The median interval between diagnosis and treatment was 3 months (IQR, 2-4). Overall, $73.2 \%$ ( $\mathrm{n}=93,764)$ of patients underwent RP within 0 to 3 months of diagnosis, $23.7 \%(\mathrm{n}=30,337)$ within 4 to 6 months, $2.5 \%(\mathrm{n}=3213)$ within 7 to 9 months, and $0.6 \%(\mathrm{n}=748)$ within 10 to 12 months.

Compared with the immediate RP group, the odds of adverse pathology were not higher with the delays of 4 to 6 months (odds ratio [OR], 0.98; 95\% CI, $0.94-1.02 ; P=.310), 7$ to 9 months (OR, 1.02 ; $95 \%$ CI, $0.91-1.13 ; P=.773)$, and 10 to 12 months (OR, 1.00; 95\% CI, 0.80-1.26; $P=.98)$. Separate subgroup analyses of men with GG 2/3 and 4/5 disease also did not show a significant difference in adverse pathology among any of the 3 delay intervals compared with immediate RP.

There was also no link between delayed RP and pathologic upgrading.
The odds of pathologic upgrading at RP were similar among the delayed RP cohorts compared with the immediate RP cohort: 4 to 6 months (OR, 1.0; 95\% CI, $0.95-1.05 ; P=.922), 7$ to 9 months (OR, $1.09 ; 95 \% \mathrm{CI}, 0.95-1.24 ; P=.228)$, and 10 to 12 months (OR, 1.06 ; 95\% CI, $0.82-1.37 ; P=.649$ ).

\section{European Study Results Align With Recommendations}

A second study, published in World Journal of Urology, analyzed the outcomes of 926 men undergoing RP for intermediate- and high-risk prostate cancer across Europe. Median follow-up and surgical delay were 26 months (IQR, 10-40) and 3 months (IQR, 2-5) respectively. No significant association between surgical delay and oncologic outcomes were found. ${ }^{2}$

The findings lend support to the current National Comprehensive Cancer Network guideline for prostate cancer management during the COVID-19 pandemic. $^{3}$

Data were retrospectively gathered from 1139 patients receiving surgery between March 2012 and September 2019 across European centers in Belgium,
France, Switzerland, and Italy. Patients in the study were categorized according to the European Association of Urology (EAU) risk categories, with time from diagnosis counted as a continuous variable.

The multivariable analysis demonstrated no significant association between surgical delay and upgrading on final specimen (OR, 0.98; 95\% CI, 0.94-1.02; $P=.3$ ), pathologically locally advanced disease (OR, 1.00; 95\% CI, 0.97-1.03; $P=.8$ ), need for adjuvant therapy (OR, 0.96; 95\% CI, 0.84-1.11; $P=.6$ ), or lymph node invasion (OR, 0.88; 95\% CI, 0.77$1.01 ; P=.07)$. The lack of impact was observed across all EAU risk categories.

While the investigators noted that most men in the study were operated on within a postdiagnosis period of close to 3 months, and they warned of extrapolating the results for much longer treatment delays, similar studies demonstrated that proposals of delays of up to 6 months for high-risk disease were reasonable, while low- and intermediate-risk groups could be delayed further.

\section{For references visit}

cancernetwork.com/delayedRP

\section{Basis for Concern \& Future Continued from page 344}

countless other cancer patients in this country and throughout the world. ${ }^{4-10}$ Many other malignancies are much faster-growing than prostate cancer, and COVID-19 delays could have serious consequences. In the urologic cancer area, testicular cancer and bladder cancer immediately come to mind as more concerning, since delay in diagnosis has been a perennial problem even in good times. ${ }^{11}$ A PubMed search of "cancer+COVID-19+delay” on August 22, 2020, found more than 125 publications, and this is just the beginning. Only time and further research will ultimately determine the long-term impact.

While I am less worried about modest delay in RP due to COVID-19, I do worry about delays in screening for prostate cancer by primary care providers that are exacerbated by COVID-19. Prostate cancer screening with the prostate-specific antigen (PSA) blood test has been controversial and hotly debated since 2011, when the US Preventive Services Task Force (USPSTF) gave PSA screening a " $\mathrm{D}$ " rating (more harm than good).12 Many advocates for patients with prostate cancer feel this rating was misguided by poor or immature data from 2 randomized controlled trials. Nevertheless, this was the "law of the land" for 8 years. In 2018, the USPSTF revised the guideline to a " $\mathrm{C}$ " rating for men aged between 55 and 69 years, citing some evidence of benefit and recognizing less overtreatment of low-risk prostate cancer, as active surveillance was more widely accepted. From 2018 to early 2020, urologists began to see more referrals from primary care providers for elevated PSA, as many began to recognize the new PSA guideline. Then COVID-19 hit. Sadly, not only did COVID strike communities of color to a greater degree, but prostate cancer is much more common in African American men. ${ }^{13}$ I worry about an “adding insult to injury" effect for Black men in this country regarding delay in 
diagnosis of prostate cancer (and many other health conditions as well). It was difficult enough to get the message out about the importance of early diagnosis of prostate cancer in the African American community, and now it's potentially much more difficult when you combine that with COVID-19.

In our practice, we began partnering with our Duke primary care network in 2018 (corresponding to the USPSTF change) to reengage prostate cancer awareness and PSA screening, focusing on our large minority high-risk population. While we lost about 3 or 4 months (March to June 2020) due to COVID-19, we are now very mindful of getting back in full swing for timely screening, urology referral, and further work-up. For men who prefer a telehealth consult to urology for elevated PSA, we now offer a novel exosome-based urine test (ExosomeDx; Bio-Techne) by mail to replace the in-person visit digital rectal examination (DRE).14,15 This new COVID-19-motivated process could tip the scales in favor of more rapid, efficient risk stratification for prostate biopsy or prostate MRI for high-risk men. Considering that the dread of a DRE prevents some men, and particularly some men of color, from going to the doctor, this new process might be a good option made possible by quick innovation indirectly caused by the COVID-19 pandemic.

In summary, cancer care is impacted by COVID-19-no question about that. Delay in diagnosis is an obvious adverse effect, deserving of further study, of which we all must be mindful, and which we must try our best to mitigate. However, out of tragedy and great adversity comes rapid advances. In addition to vaccines and effective therapies for COVID-19, we in the oncology field must strive to use this pandemic to improve our care of cancer over the long term, and to think outside the box.

For references visit

cancernetwork.com/Moul_commentary

\section{We Are Oncology.}
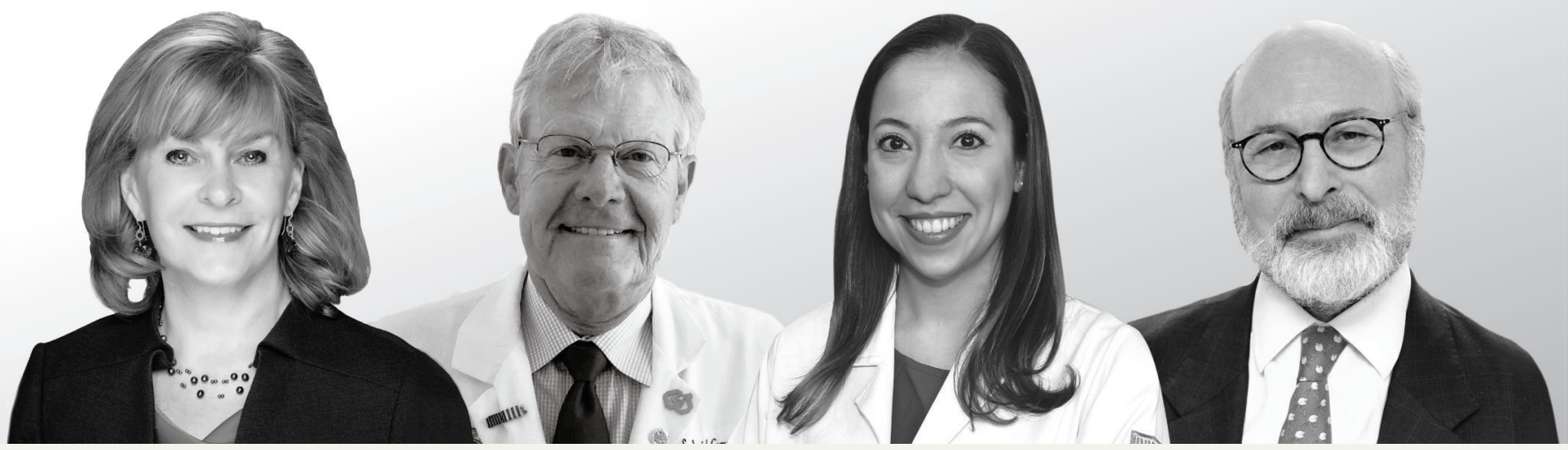

Peer review and perspective. More context-deeper insight. Explore us online.

\section{go to cancernetwork.com}




\section{Recurrent EGFR-Mutated Non-Small Cell Lung Cancer Discovered by Abnormal Mammogram: Adjuvant/ Frontline Metastatic Management Options}

Mehmet Sitki Copur, MD; Rudy Lackner, MD²; Paul Rodriguez, MD³; Adam J. Horn, MD'; Shellie Faris, MD5; and Thomas Zusag, MD'

'Morrison Cancer Center, Mary Lanning Healthcare, Hastings, NE; ' 2 Section of Thoracic Surgery, University of Nebraska Medical Center, Omaha, NE; ${ }^{3}$ Radiology Department, Mary Lanning Healthcare, Hastings, NE; ${ }^{4}$ Pathology Department, Mary Lanning Healthcare, Hastings, NE; ${ }^{5}$ Surgery Department, Mary Lanning Healthcare, Hastings, NE.

ABSTRACT: Breast metastasis from extramammary malignancy is rare, with a reported incidence rate of $0.4 \%$ to $1.3 \%$ in the published literature. The primary malignancies that most commonly metastasize to the breast are leukemia, lymphoma, and malignant melanoma. Here, we report a very rare case of metastatic EGFR-mutated non-small cell lung cancer (NSCLC) in the breast detected by screening mammography. The patient had initially been diagnosed with a clinical stage IIIA NSCLC and had been treated with neoadjuvant chemoradiation followed by curative-intent surgery. Several interesting aspects of the case, along with a discussion of evolving adjuvant and frontline metastatic management options in EGFR-mutated NSCLC, will be presented.

\section{Introduction}

Lung cancer is the leading cause of cancer mortality in both men and women, and the second most common cancer in both genders. ${ }^{1}$ Non-small cell lung cancer (NSCLC) accounts for $80 \%$ to $90 \%$ of all lung cancer cases, half of which present with adenocarcinoma histology. ${ }^{2}$ In the past decade, several predictive and prognostic biomarkers have become available, significantly changing the treatment landscape in this disease. Current guidelines recommend testing for certain molecular and immune biomarkers in the metastatic setting to assess eligibility for targeted or immunotherapy options. ${ }^{3-5}$ EGFR driver mutations are the most common predictive biomarkers. Among others with an FDA approved targeted therapy are $A L K$ fusion oncogene, ROS1 gene fusions, BRAF V600E point mutations, NTRK gene fusions, METex14 skipping mutations, RET rearrangements, and
PD-L1 expression. Somatic activating mutations in the tyrosine kinase (TK) domain of EGFR behave as oncogenic drivers leading to ligand independent activation of downstream receptor signaling, favoring cell proliferation, survival, and migration. ${ }^{6}$

The mostcommon EGFR gene mutations in patients with NSCLC are deletions in exon 19 (exon 19del) and a point mutation in exon 21 (L858R), accounting for $45 \%$ and $40 \%$ of all patients, respective- 

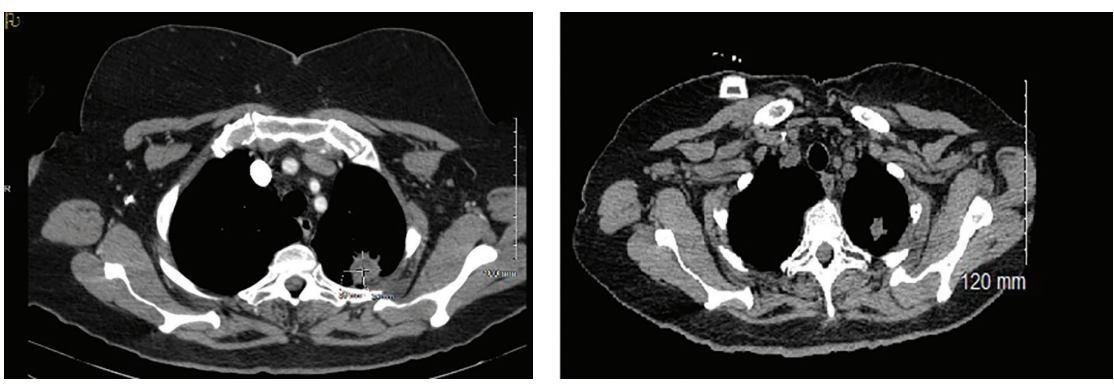

- Figure 1. CT scan through the lung apices shows a 3.1-cm spiculated mass with central low density consistent with necrosis. At the level of the left upper aorticopulmonary window region, there is a 3.0-cm nodal soft tissue mass with central density consistent with necrosis.
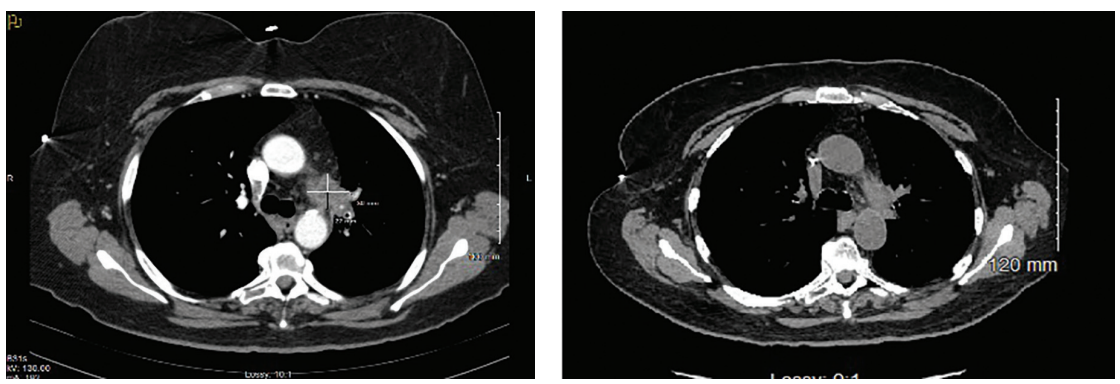

- Figure 2. CT scan after treatment shows significant reduction of the left apical pulmonary mass and the left upper hilar nodal mass, consistent with a positive response to chemoradiation.

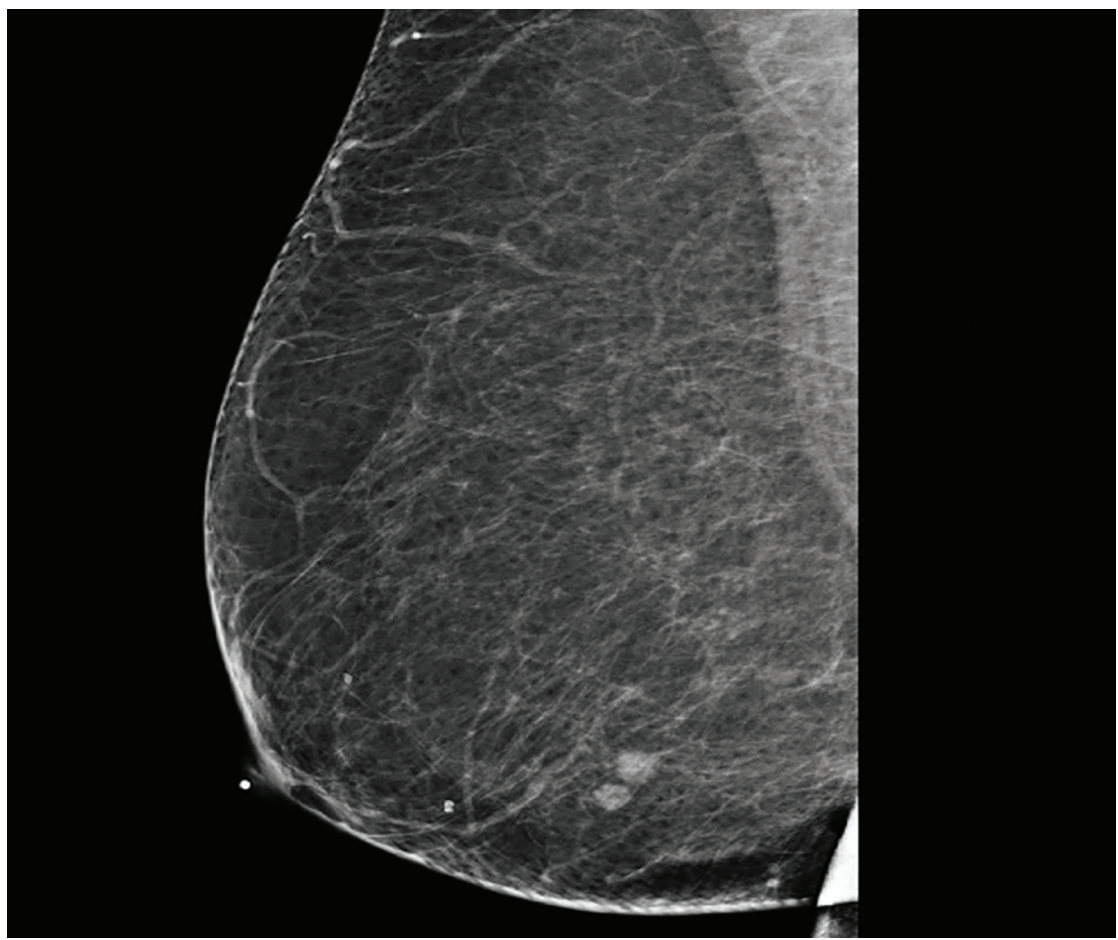

- Figure 3. Right mediolateral oblique digital mammogram view shows 2 adjacent nodules in the lower breast. Margins are slightly lobulated. The finding was new from the prior mammogram and suspicious for malignancy. ly. Both mutations result in activation of the TK domain, and both are associated with sensitivity to the small molecule EGFR TK inhibitors (TKIs), such as erlotinib, gefitinib, afatinib, osimertinib, and dacomitinib. ${ }^{7}$ These drug-sensitive EGFR mutations have been named as sensitizing mutations. Other less common mutations $(5 \%-10 \%)$ that are also sensitive to EGFR TKIs include exon 19 insertions, p.L861Q, p.G719X, and p. S768I. ${ }^{8}$ Overall, these sensitizing EGFR mutations are found in $10 \%$ of Caucasian patients and up to $50 \%$ of Asian patients with NSCLC. ${ }^{9}$

Here, we review the rare occurrence of breast metastases from EGFR-mutated NSCLC and discuss the recent developments in the adjuvant and metastatic management of this disease.

\section{Case}

A 68-year-old Caucasian woman, lifelong nonsmoker, presented with recurring cough and upper respiratory infection symptoms. Despite initial improvement with antibiotics at each episode, the recurrent nature of her symptoms led to further work-up. A CT scan of her chest revealed a left apical pulmonary mass measuring up to $2.6 \times 3.1 \mathrm{~cm}$ and a left aorticopulmonary window mediastinal lymph node measuring 2.7 x $3.0 \mathrm{~cm}$ (Figure 1). CT-guided biopsy of the lung lesion revealed an adenocarcinoma consistent with lung primary. Further work up with a PET-CT scan confirmed the left apex lung mass with a maximum standardized uptake value (SUV) of 6.4 and the left aorticopulmonary window lymph node with a maximum SUV of 6.7. There were no other hypermetabolic areas. She was staged as cT2 cN2cM0, stage IIIA.

Preoperative chemoradiation (pemetrexed $500 \mathrm{mg} / \mathrm{m}^{2}$ plus cisplatin $75 \mathrm{mg} /$ $\mathrm{m}^{2}$ with concurrent radiation [45 Gy in 180 cGy daily fractions]) led to a decrease in the left apex mass down to $1.7 \times 1.4$ $\mathrm{cm}$, and only minimal soft tissue fullness remained in the left aorticopulmonary 


\section{TABLE. Adjuvant Immunotherapy Trials for NSCLC}

\begin{tabular}{llllll}
\hline Trial identifier & Immunotherapy & End point & Stage & Phase & Sponsor \\
NCT03053856 & Pembrolizumab & DFS & IIIA & 2 & Samsung Medical Center \\
\hline NCT02486718 & Atezolizumab & DFS & IB-IIIA & 3 & Hoffmann-La Roche \\
\hline NCT03130764 & Durvalumab and tremelimumab & T-cell response & IB-IIIA & 2 & Columbia University \\
\hline NCT02595944 & Nivolumab & DFS; OS & IB-IIIA & 3 & National Cancer Institute \\
\hline NCT02273375 & Durvalumab & DFS & IB-IIIA & 3 & Canadian Cancers Trials Group \\
\hline NCT02504372 & Pembrolizumab & - & IB-IIIA & 3 & European Platform of Cancer \\
\end{tabular}

DFS, disease-free survival; OS, overall survival.

window lesion (Figure 2). She underwent a left video-assisted thoracoscopic surgery with an upper lobectomy and mediastinal lymph node sampling. Pathology revealed left upper lobe $1.6-\mathrm{cm}$ mass consisting of fibroelastosis chronic inflammation macrophage infiltration and reactive epithelial changes consistent with pulmonary tissue with therapy effect, but no residual viable tumor. Similar changes with areas of dense fibrosis and chronic hemorrhage were identified in the level 5 mediastinal node specimen, but no nodal malignancy involvement, confirming complete pathologic response (pyT0pyN0).

Six months following her surgery, a routine annual screening mammogram revealed 2 new adjacent nodules in the lower central right breast suspicious for breast cancer (Figure 3). Ultrasound guided biopsy of these lesions revealed adenocarcinoma consistent with metastasis from lung primary (Figure 4). A restaging PET-CT scan revealed new hypermetabolic lesions involving caudate lobe of liver, left adrenal gland, and bone lesions in left humerus, right scapula, and right ischium, which were totally asymptomatic (Figure 5).

\section{Discussion}

This case illustrates not only a very unusual initial metastatic site for a recurrent EGFR-mutated NSCLC, but it also stimulates a discussion on several evolving management aspects in the adjuvant
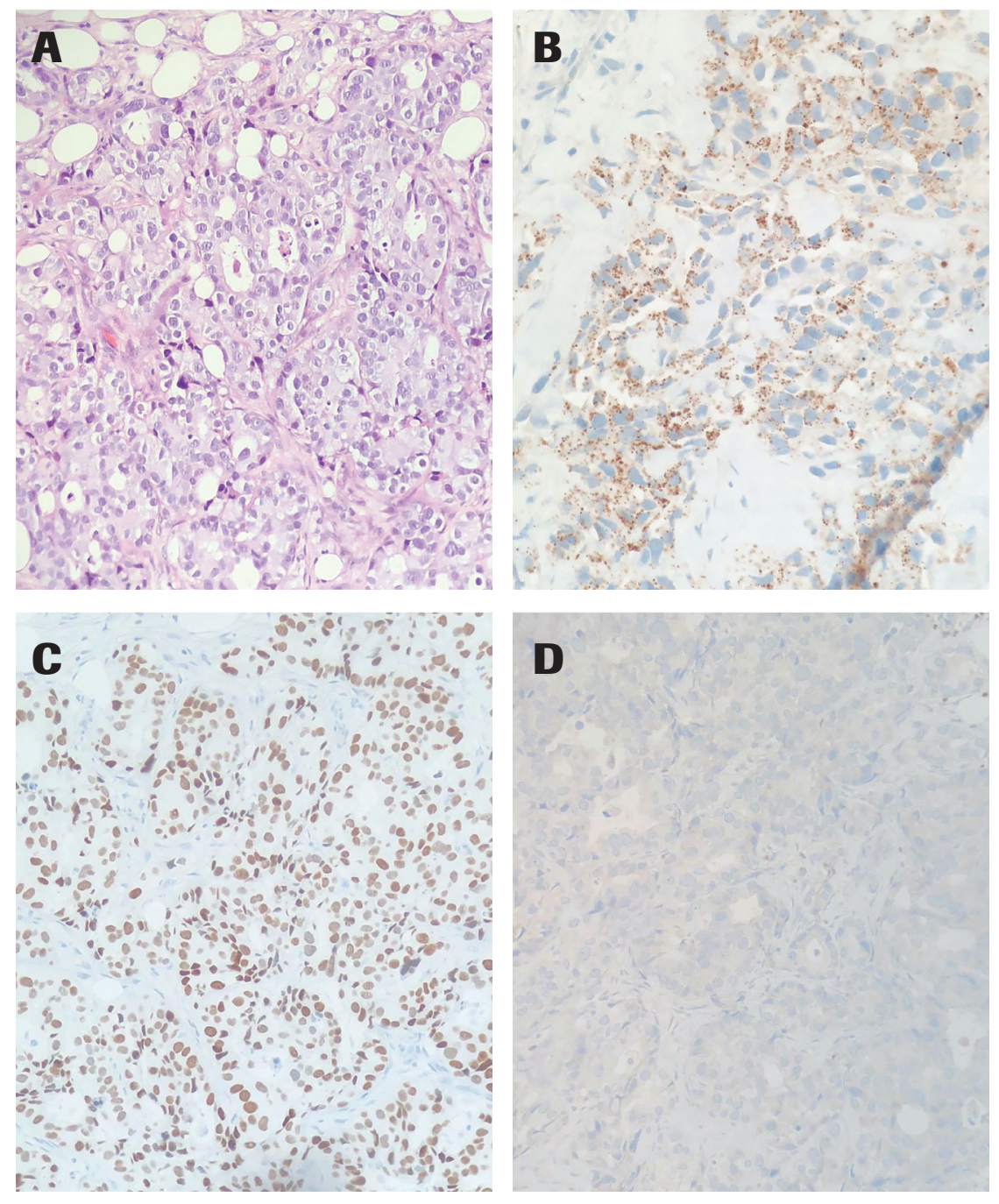

Figure 4. Breast biopsy, invasive adenocarcinoma (hematoxylin and eosin stain). (A); Breast biopsy, invasive adenocarcinoma. Napsin A immunohistochemical stain (cytoplasmic positivity). (B); Breast biopsy, invasive adenocarcinoma. TTF-1 immunohistochemical stain (nuclear positivity). (C); Breast biopsy, Invasive adenocarcinoma. GATA3 immunohistochemical stain (negative). 

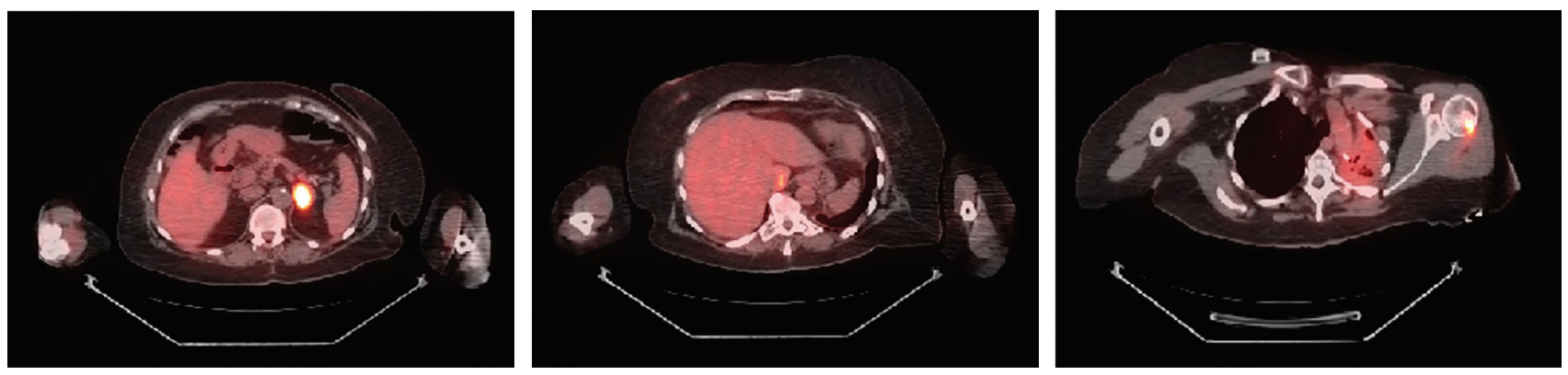

- Figure 5. New PET-CT scan. New positive areas of F-18 fluorodeoxyglucose uptake in the left adrenal gland, caudate lobe of the liver, and left humerus. Vague, minimal uptake in the left lung was thought to be pneumonitis.

and metastatic setting of EGFR-mutated NSCLC.

The most common sites of metastasis from NSCLC are liver (33\%-40\%), adrenal glands $(18 \%-38 \%)$, brain $(15 \%$ $43 \%)$, bone (19\%-33\%), kidney (16\%$23 \%)$, and abdominal lymph nodes $(29 \%) \cdot{ }^{10}$ However, an autopsy series has demonstrated that NSCLC may spread virtually to any organ. ${ }^{11}$ The mammary gland is a rare site of involvement by metastatic disease from extramammary malignancies, likely due to its large areas of fibrous tissue with a relatively poor blood supply. The incidence of breast metastases from extramammary malignancies has been reported to be around $0.4 \%$ to $1.3 \%$ with a wide range of primary malignant tumors including hematological malignancies, malignant melanoma, lung cancer, renal cancer, ovarian cancer, thyroid carcinomas, and small bowel carcinoids. ${ }^{12-15}$

Among the extramammary malignancies metastasizing to the breast, NSCLC is very rare, with a total of 12 cases reported between 1992 and 2012 in the PubMed database. ${ }^{13,16-24}$ In a further updated systematic review of the literature, Mirrielees et a ${ }^{25}$ identified 31 case reports of NSCLC and 8 cases of small cell lung cancer (SCLC) metastatic to the breast.

Although breast metastases from primary lung cancer are rare, EGFR-mutated lung cancers metastasizing to the breast are even rarer, with a total of 5 cases reported to date. Fukumoto et $\mathrm{a}^{26}$ reported a case of late breast metastasis from a previously resected EGFR-mutated lung adenocarcinoma. Sato et $\mathrm{al}^{27}$ reported the case of a woman who was initially treated with first-line gefitinib for an EGFR-mutated lung adenocarcinoma. After 12 months of gefitinib, she developed breast metastasis. The metastatic tumor tissue tested positive for T790M mutation. Dansin et al ${ }^{28}$ reported a case of a woman, aged 52 years, with a breast mass initially diagnosed as a primary triple negative breast cancer; this later proved to be a metastasis from an EGFR-mutated lung adenocarcinoma. Ota et a ${ }^{29}$ reported a case of metastasis to the breast from a primary lung adenocarcinoma with an L858R mutation in exon 21. Finally, a case of SCLC metastatic to the breast, which transformed from NSCLC during EGFR-TKI maintenance therapy, was reported. ${ }^{30}$ Our case is thus the sixth reported of EGFR-mutated NSCLC metastatic to the breast.

Initial clinical presentation of our case was clinical stage IIIA. The optimal management of patients with potentially operable stage IIIA NSCLC remains controversial. Two randomized trials evaluated the role of surgery in this setting, neither showing an overall survival (OS) benefit. ${ }^{31-33}$ This is most likely due to the heterogeneity of the patient population with $\mathrm{N} 2$ disease in these trials. Similarly, there is no consensus on the role of neoadjuvant chemoradiotherapy vs neoadjuvant chemotherapy alone. OS appears to be similar as long as radiation therapy is given postoperatively, if not given preoperatively. ${ }^{34,35}$ However, neoadjuvant chemoradiotherapy has been associated with higher rates of pathologic complete response rates and negative mediastinal lymph nodes. ${ }^{36}$ For patients with unresectable stage III NSCLC, the recommendation is definitive concurrent chemoradiation followed by immunotherapy with durvalumab as consolidation therapy, based on the data from a phase 3 trial. ${ }^{37}$ On the contrary, there are no established data for the use of adjuvant durvalumab or any other immunotherapy options in resected stage IIIA NSCLC patients. Several ongoing clinical trials are currently testing this concept (Table). ${ }^{37,38}$ Our patient was treated with neoadjuvant chemoradiation followed by surgery. Despite a complete pathologic response, the unfortunate manifestation of recurrent disease raises the question of possible benefit from adjuvant immunotherapy in this setting.

Once recurrent metastatic disease was established in the patient in our case, molecular and immune biomarker testing was performed, which revealed the EGFR mutation (exon 19del). This finding raises 3 other important management questions: the role of testing for molecular and immune biomarkers of tumor tissue in the adjuvant setting; the role of adjuvant TKI treatment in resected EGFR-mutated NSCLC; and the optimal first-line targeted treatment for 
metastatic EGFR-mutated NSCLC.

Earlier trials have not demonstrated any benefit of TKI use in the adjuvant setting. However, recent randomized controlled trial results have shown a disease-free survival (DFS) advantage. In a systematic review and meta-analysis of 6 trials incorporating 1860 patients, adjuvant TKIs decreased the risk of disease recurrence by $48 \%$; they improved 2 -year DFS but not OS. In subgroup analyses, the DFS benefit was more pronounced in the trials using TKIs rather than chemotherapy, as opposed to trials using TKIs sequentially after chemotherapy. ${ }^{39}$ In another meta-analysis involving 1960 patients who participated in 5 studies, adjuvant EGFR-TKI treatment was associated with an absolute DFS benefit of $3.1 \%$ at 3 years. Finally, in an additional study, adjuvant EGFR-TKI treatment was associated with a marginally statistically significant benefit in OS. ${ }^{40}$

Most recently, the first interim analysis from the phase 3 ADAURA trial showed $83 \%$ improved overall DFS vs placebo (<.0001). In stage II to IIIA NSCLC patients treated with osimertinib vs placebo, the 2-year DFS was 90\% vs $44 \%$, respectively. ${ }^{41}$ Osimertinib is currently approved for first-line treatment of EGFR-mutated advanced NSCLC and for second-line treatment of EGFR-T790M-mutated advanced NSCLC. The role of TKIs in the adjuvant treatment of EGFR-mutated NSCLC re- mains hotly debated. Long-term survival data on this topic are eagerly waited.

The optimal first-line treatment regimen for patients with advanced metastatic EGFR-mutated NSCLC is also not well established. A meta-analysis of 25 randomized clinical trials with a total of 5005 patients showed that either the third-generation TKI osimertinib, or first-generation TKIs in combination with chemotherapy, were more effective than first-generation TKIs alone in terms of progression-free survival (PFS) and OS. Second-generation TKIs show significant OS advantage over first-generation TKIs. Based on PFS and OS, osimertinib has the highest probability of being the most effective treatment. ${ }^{42}$

Finally, the recently published results of the phase 3 RELAY trial, which compared ramucirumab plus erlotinib against erlotinib alone, showed superior median PFS for the combination (19.4 months) vs erlotinib alone (12.4 months). ${ }^{43}$ While the OS data will not be mature until 2023, statistically projected and calculated OS data were reported to be 58 months for ramucirumab/erlotinib vs 52 months for erlotinib alone.

\section{Outcome}

After a discussion of different treatment options, our patient opted for treatment with the ramucirumab/erlotinib combination. Currently she is receiving her second cycle and tolerating treatments well. A restaging scan is planned in the near future.

\section{FINANCIAL DISCLOSURE: The authors have} no significant financial interest in or other relationship with the manufacturer of any product or provider of any service mentioned in this article.

\section{KEY REFERENCES}

1. American Cancer Society Cancer Facts \& Figures: 2020. American Cancer Society. 2020. Accessed September 1, 2020. https://www. cancer.org/content/dam/cancer-org/research/ cancer-facts-and-statistics/annual-cancerfacts-and-figures/2020/cancer-facts-andfigures-2020.pdf

2. Li T, Kung HJ, Mack PC, Gandara DR. Genotyping and genomic profiling of non-smallcell lung cancer: implications for current and future therapies. J Clin Oncol. 2013;31(8):10391049. doi:10.1200/JCO.2012.45.3753

3. Ramalingam SS, Vansteenkiste J, Planchard D, et al; FLAURA Investigators. Overall survival with osimertinib in untreated, EGFR-mutated advanced NSCLC. N Engl J Med. 2020;382(1):41-50. doi:10.1056/ NEJMoa1913662

4 Garon EB, Hellmann MD, Rizvi NA, et al. Five-year overall survival for patients with advanced non-small-cell lung cancer treated with pembrolizumab: results from the phase I KEYNOTE-001 study. J Clin Oncol. 2019;37(28):2518-2527. doi:10.1200/ JCO.19.00934

5. Lin JJ, Cardarella S, Lydon CA, et al. Five-year survival in EGFR-mutant metastatic lung adenocarcinoma treated with EGFRTKIs. J Thorac Oncol. 2016;11(4):556-565. doi:10.1016/j.jtho.2015.12.103

\section{For full reference list, visit:} cancernetwork.com/EGFR_NSCLC

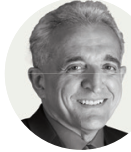

\section{Copur}

is a medical oncologist/ hematologist at Morrison Cancer Center, Mary Lanning Healthcare in Hastings, Nebraska, and is a professor at the University of Nebraska Medical Center. He is also an Editorial Advisory Board member at ONCOLOGY ${ }^{\circledR}$.

\section{Horn}

is a pathologist at Mary Lanning Healthcare in Hastings, Nebraska.

\section{Lackner}

(19) is currently the vice chief of Cardiothoracic Surgery and chief of the section of Thoracic Surgery. $\mathrm{He}$ is also the director of the Comprehensive Thoracic Oncology Program at the University of Nebraska Medical Center.

\section{Faris}

is a surgical oncologist at Mary Lanning Healthcare in Hastings, Nebraska.

\section{Rodriguez}

is a board certified diagnostic radiologist at Mary Lanning

Healthcare in Hastings, Nebraska and vice president of Hastings Radiology Associates. 


\section{Molecular Profiling in Metastatic Colorectal Cancer}

Samantha A. Armstrong, MD; Rita Malley, MS; and Benjamin A. Weinberg, MD

Ruesch Center for the Cure of Gastrointestinal Cancers, Lombardi Comprehensive Cancer Center, Washington, DC

\begin{abstract}
Colorectal cancer (CRC) is a commonly diagnosed malignancy. Although chemotherapy remains the backbone of treatment, the landscape of treating metastatic $\mathrm{CRC}$ ( $\mathrm{mCRC}$ ) is changing with the understanding of its heterogeneity and molecular blueprint. Colon cancer sidedness has proven to hold prognostic implications, with right-sided tumors having higher incidence of $B R A F$ and $K R A S$ mutations and being microsatellite instabilityhigh (MSI-H); overall, they have a worse prognosis compared with left sided-tumors. Results of molecular research have demonstrated the need to profile each mCRC patient for RAS and BRAF mutations, MSI-H status, HER2 amplifications, and NTRK fusions. Ongoing clinical trials using targeted agents aim to further improve survival outcomes. We emphasize the epidemiology, knowledge of primary tumor location, and mutational landscape of mCRC, as well as novel treatment options for patients harboring unique subtypes of these characteristics.
\end{abstract}

\section{Introduction}

Colorectal cancer (CRC) is a commonly diagnosed malignancy and the third leading cause of cancer death in the United States, with 147,950 estimated new cases and 53,200 estimated deaths in 2020. ${ }^{1}$ Despite improvements in CRC screening methodologies, approximately $20 \%$ of patients are diagnosed with metastatic CRC (mCRC), which carries a $14.2 \%$ 5-year survival rate. ${ }^{2}$ Although patients with stage IV CRC can occasionally undergo curative metastasectomies, treatment of patients with $\mathrm{mCRC}$ is for the most part palliative in nature and aims to prolong and improve the quality of life. A better understanding of the heterogeneity of mCRC, including primary tumor location, microsat- ellite instability (MSI) status, and other clinically actionable tumor mutations, is reshaping the therapeutic landscape. Here we review the current molecular profiling practice for mCRC along with its prognostic implications and potential therapeutic targets.

\section{Colon Cancer Sidedness}

Colon tumor sidedness has been a topic of great interest. The activated pathways in mCRC differ between the left and right sides. ${ }^{3}$ The difference in sidedness may stem from embryonic origin: the right side of the colon arises from the midgut, whereas the left side arises from the hindgut, affecting blood supply. ${ }^{4}$ Differences in gut microbiome, related to increased bile acid exposure on the right

\section{PERSPECTIVE}

John L. Marshall, MD on transforming global cancer care page 356

side and predominant bacteria on the 2 sides of the colon-including Prevotella, Pyramido-bacterium, Selenomonas, and Peptostreptococcus (right); and Fusobacterium, Escherichia-Shigella, and Leptotrichia (left)-may all play a role in the phenotype of CRC sidedness. ${ }^{4}$ The right side of the colon includes the cecum through to the proximal two-thirds of the transverse colon, and the left side of the colon includes the distal third of the transverse colon through to the sigmoid colon. ${ }^{4}$ Most studies define right-sided tumors as those that arise from the cecum through but not including the splenic flexure, and left-sided tumors are those found in the splenic flexure through the sigmoid colon. ${ }^{5}$ Rectal cancers are frequently included with left-sided colon cancers for the purpose of sidedness analyses.

The results of many studies have shown that patients with left-sided colon tumors tend to have superior overall survival (OS) compared with patients with right-sided tumors. ${ }^{6-10}$ The CALGB/ SWOG 80405 trial results revealed that the addition of cetuximab (an anti-EGFR monoclonal antibody) vs bevacizumab (an anti-VEGF monoclonal antibody) to standard chemotherapy in the first-line treatment of patients with KRAS exon 2 
TABLE. Alterations, Frequency, Sidedness, and Targeted Therapies

\begin{tabular}{|c|c|c|c|c|c|}
\hline Alteration & Frequency & Sidedness & $\begin{array}{l}\text { Therapeutic agents if } \\
\text { altered }\end{array}$ & $\begin{array}{l}\text { Therapeutic } \\
\text { agents if WT }\end{array}$ & Ongoing clinical trials \\
\hline \multirow[t]{3}{*}{ KRAS mutation } & \multirow[t]{3}{*}{$40 \%-50 \%$} & \multirow[t]{3}{*}{ Right } & \multirow[t]{3}{*}{-} & \multirow[t]{3}{*}{$\begin{array}{l}\text { EGFR inhibitor } \\
\text { (cetuximab, }^{\text {panitumumab) }}\end{array}$} & $\begin{array}{l}\text { G12C mutation: NCT04006301 } \\
\text { NCT03785249 } \\
\text { NCT04165031NCT04185883 } \\
\text { NCT03114319 }\end{array}$ \\
\hline & & & & & G12D mutation: NCT03745326 \\
\hline & & & & & $\begin{array}{l}\text { G12V mutation: } \\
\text { NCT03190941 }\end{array}$ \\
\hline NRAS mutation & $4 \%-5 \%$ & - & - & $\begin{array}{l}\text { EGFR inhibitor } \\
\text { (cetuximab, panitu- } \\
\text { mumab)a }\end{array}$ & - \\
\hline $\begin{array}{l}\text { BRAF V600E } \\
\text { mutation }\end{array}$ & $8 \%-10 \%$ & $\begin{array}{l}\text { Class } 1 \text { right } \\
\text { Class 2-3 left }\end{array}$ & $\begin{array}{l}\text { Vemurafenib; dabrafenib; } \\
\text { trametinib; encorafenib + } \\
\text { binimetinib }\end{array}$ & $\begin{array}{l}\text { EGFR inhibitor } \\
\text { (cetuximab, panitu- } \\
\text { mumab)a }\end{array}$ & - \\
\hline HER2 & $5 \%-26.7 \%$ & Rectum/left & $\begin{array}{l}\text { Trastuzumab }{ }^{c} \text {; lapatinibc; } \\
\text { pertuzumab }^{c} \text {; tucatinib }\end{array}$ & - & $\begin{array}{l}\text { NCT03365882 } \\
\text { NCT03043313 }\end{array}$ \\
\hline NTRK fusion & $1 \%$ & - & Larotrectinib; entrectinib & - & NCT03215511 \\
\hline $\begin{array}{l}\text { dMMR/MSI high/ } \\
\text { TMB }^{\text {b }}\end{array}$ & - & Right & $\begin{array}{l}\text { Nivolumab +/- ipilimumab; } \\
\text { pembrolizumab }^{\mathrm{d}}\end{array}$ & - & $\begin{array}{l}\text { NCT02997228 } \\
\text { NCT02912559 } \\
\text { NCT02563002 }\end{array}$ \\
\hline
\end{tabular}

dMMR, mismatch repair deficient; MSI, microsatellite instability; TMB, tumor mutational burden; WT, wild type.

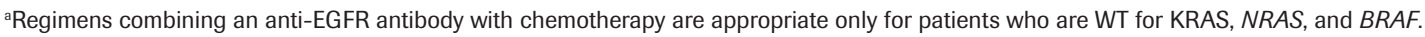

${ }^{\mathrm{b} C o m b i n a t i o n s ~ o f ~ e n c o r a f e n i b ~ p l u s ~ c e t u x i m a b ~ o r ~ p a n i t u m u m a b ~ a r e ~ a p p r o p r i a t e ~ f o r ~ p a t i e n t s ~ w i t h ~} B R A F$ V600E mutations.

${ }^{c}$ Combinations of trastuzumab plus pertuzumab or lapatinib are appropriate only for patients with $H E R 2$-amplified and wild-type $R A S$ and $B R A F$.

dPembrolizumab use is for TMB-high patients.

wild-type (WT) mCRC did not improve OS, necessitating further study by dividing these patients into subsets to discover how best to direct standard therapeutic recommendations. ${ }^{11} \mathrm{~A}$ follow-up study utilizing the same patient population $(\mathrm{N}=$ 782) confirmed primary tumor location as an independent prognostic factor for OS, with right-sided tumors being associated with inferior survival (HR, 1.392; 95\% CI, 1.032-1.878; $P=.031) .{ }^{11}$ Right-sided compared with left-sided colorectal tumors were found to have a higher $B R A F$ mutation frequency and were more likely to be MSI-high (MSI-H). ${ }^{12,13}$

The NCIC CO.17 trial $(\mathrm{N}=399)$ demonstrated that right-sided colon tumors ( $\mathrm{N}=150)$ were more poorly differentiated and had a higher rate of KRAS mutations than left-sided tumors. ${ }^{5}$ For patients with KRAS WT tumors only, those with left-sided tumors had an increased progression-free survival (PFS) benefit from cetuximab compared with best supportive care (median PFS, 5.4 vs 1.8 months; HR, 0.28 ; $P<.0001$ ), whereas those with right-sided tumors received no such benefit (median 1.9 vs 1.9 months; HR, $0.73 ; P=.26) .{ }^{5}$ Investigators from other trials also identified a response to chemotherapy plus anti-EGFR therapy in patients with left-sided $B R A F / R A S$ WT tumors, while those with right-sided tumors had adverse outcomes to anti-EGFR therapy but benefited from chemotherapy plus VEGF inhibition. , $^{5,11,14-16}$

\section{RAS Mutations}

Mutations in any 1 of 3 RAS oncogenes-KRAS, NRAS, and HRAShave been found to lead to autonomous growth and differentiation of cells driving cancer progression. A recent study of CRC patients $(\mathrm{N}=13,336)$ identified KRAS mutations in $51.9 \%$ of specimens, with NRAS and HRAS mutations occur- ring at lower rates. ${ }^{17}$ These study results revealed that KRAS-mutated colorectal tumors were less likely to be mismatch repair deficient (dMMR). ${ }^{17}$

The presence of KRAS or NRAS mutations correlates with worse outcomes and resistance to anti-EGFR therapy, with or without chemotherapy. ${ }^{17-22}$ Investigators in the phase 3 PRIME trial $(\mathrm{N}=1183)$ randomized patients with treatment-naïve mCRC to receive panitumumab plus FOLFOX (5-FU, leucovorin, and oxaliplatin) or FOLFOX alone. ${ }^{23}$ This study demonstrated that in patients with KRAS/NRAS WT disease, the addition of panitumumab to FOLFOX resulted in superior median PFS (10.1 vs 7.9 months; HR, 0.72; $P=.004)$ and median OS (26.0 vs 20.2 months; HR, $0.78 ; P=.04)$. BRAF mutations were a negative prognostic and predictive factor, as patients with BRAF mutations did not benefit from panitumumab. ${ }^{23}$ 
$R A S$ mutations, specifically KRAS and NRAS mutations, are used as predictive biomarkers for therapeutic resistance to upstream EGFR inhibitors. However, the successful development of targeted therapies to KRAS and other downstream signaling proteins means that KRAS mutation testing may play another role. Multiple phase 1 trials are testing small molecule agents that target the KRAS glycine-to-cysteine (G12C)mutated protein (NCT04006301, NCT03785249, NCT04165031, NCT04185883). In addition to direct mutational targeting, agents targeting SHP2-which, encoded by PTPN11, is needed to activate RAS-MAPKmay become a viable therapeutic option for KRAS G12C-mutated CRC (NCT03114319). Additional targeted KRAS therapies include lymphocytes genetically engineered to express murine T-cell receptors that recognize G12V and G12D mutations (NCT03745326, NCT03190941).

\section{BRAF Mutations}

Similar to the effects of KRAS mutation, patients with $B R A F$-mutated mCRC do not respond to anti-EGFR monotherapy (panitumumab or cetuximab) and have shorter PFS and OS compared with patients with BRAF WT tumors. ${ }^{19,24}$ Approximately $8 \%$ to $10 \%$ of mCRCs contain a mutation in $B R A F$, a gene that encodes a signal transduction protein that is involved in the MAPK pathway. ${ }^{25,26}$

Due to its link to poor patient prognosis, the BRAF V600E mutation is a worthy target in CRC. However, BRAF inhibition alone has limited efficacy in the treatment of patients with mCRC. Vemurafenib is a small molecule tyrosine kinase inhibitor (TKI) that specifically targets the ATP-binding domain of BRAF V600E, hence activating the MAPK pathway in advanced malignancies with BRAF V600E mutations. ${ }^{26}$ In mCRC, vemurafenib monotherapy is rarely effective due to incomplete inhibition of MAPK signaling and a re- flexive activation of EGFR. ${ }^{26}$ Kopetz and colleagues studied the efficacy of irinotecan plus cetuximab with or without vemurafenib in 106 patients with refractory $B R A F$-mutated mCRC. ${ }^{27} \mathrm{PFS}$ was improved with the addition of vemurafenib (median 4.4 vs 2.0 months; $P<.001)$, and there was a trend toward increased response rate (16\% vs $4 \%$ ) and disease control rate $(67 \%$ vs $22 \%)$. Hence, vemurafenib plus irinotecan and cetuximab provided clinical benefit and became a new treatment option for this subset of patients. ${ }^{27}$

In addition to combined inhibition of EGFR and BRAF, the inhibition of the MAPK pathway has been explored through the combined inhibition of MEK, EGFR, and BRAF. The phase 3 BEACON study randomized patients with previously treated BRAF V600Emutated mCRC 1:1:1 to triplet therapy of encorafenib (a BRAF inhibitor), cetuximab, and binimetinib (a MEK inhibitor); doublet therapy with encorafenib and cetuximab; or the control arm of chemotherapy (FOLFIRI or irinotecan) with cetuximab $(\mathrm{N}=665) .{ }^{28}$ Patients treated with the triplet and doublet had significant longer median OS when compared with the chemotherapy arm: 9.3 and 9.3 vs 5.9 months, respectively. Objective response rate (ORR) was also higher with the triplet $(27 \%)$ and doublet $(20 \%)$ compared with the chemotherapy arm $(2 \% ; P<.0001) .{ }^{28}$ As there was not a statistical numerical difference in terms of OS between the triplet and doublet, encorafenib and cetuximab (without binimetinib) was approved by the FDA. ${ }^{29}$ The ongoing phase 3 ANCHOR trial is evaluating the triplet in the first-line setting.

\section{MSI, TMB, PD-L1}

The PD-1 pathway is a negative feedback system that regulates cytotoxic Th1 immune response to prevent damage to host cells. ${ }^{30}$ Tumor cells upregulate the PD-1 receptor or PD-1 ligand (PD-L1 or PD-L2) to evade destruction by the host's immune system. The PD-1 pathway has been exploited in cancer immunotherapy. By blocking the PD-1 pathway with antibodies to PD-1 or PD-L1/PD-L2, the adaptive immune response is activated against tumor cells, resulting in an anticancer response. This has been observed in patients with a number of advanced cancers, including melanoma, non-small cell lung cancer, renal cell carcinoma, and others. ${ }^{30}$ Further exploration of the PD-1 pathway has demonstrated the connection between response to PD- 1 immune checkpoint inhibitors and dMMR. CRC with dMMR or MSI-H have a poor response to chemotherapy regimens, resulting in a worse prognosis for patients with metastatic disease. $^{31}$

Tumors with dMMR contain large numbers of mutation-associated neoantigens; the immune system is more likely to recognize these antigens as foreign and launch an immune response. The clinical benefit of PD-1 blockade with pembrolizumab (an anti-PD-1 antibody) was first documented in a phase 2 study $(\mathrm{N}=41)$, which enrolled patients with metastatic carcinoma with and without $\mathrm{dMMR}$, and results revealed that only those with dMMR benefit from PD-1 blockade. Eleven patients with dMMR had CRC: 4 patients achieved a partial response and 5 had stable disease. ${ }^{30}$ In a larger study of this population, the ORR of patients with dMMR CRC $(\mathrm{N}=40)$ was $52 \%$, with a 2 -year PFS of $59 \% .32$ Of the posttreatment biopsies obtained and analyzed $(\mathrm{N}=20)$, 12 showed inflammation, fibrosis, and mucin, but no evidence of tumor cells. This demonstrated an immunological anticancer response to PD-1 inhibitor therapy. ${ }^{32}$ The phase 2 KEYNOTE-164 trial evaluated pembrolizumab in larger cohort of 124 patients with metastatic MSI-H/dMMR mCRC and demonstrated an ORR of $33 \% .{ }^{33}$ Additionally, analysis of tumor mutational burden in the phase 2 KEYNOTE-158 trial led to the FDA approval of pembrolizumab across solid tumors harboring a tumor 
mutation burden (TMB) of 10 or more mutations/megabase after progression on prior therapy and showed a response rate of $29 \% .^{34,35}$

The anti-PD-1 antibody nivolumab was also found to be an effective therapy for patients with MSI-H/dMMR mCRC. Investigators of the CheckMate 142 trial $(\mathrm{N}=74)$ reported ORRs, by RECIST 1.1 , of $31.1 \%$. Disease control rate at $>12$ weeks was found to be $68.9 \% .^{31}$ Of note, tumor expression of PD-L1 was not found to be predictive of response because an objective response and disease control were also found in subjects with and without PD-L1 expression. ${ }^{31}$ Additionally, nivolumab and other checkpoint inhibitors may exhibit greater activity than conventional chemotherapy or targeted therapy in patients with MSI-H, BRAF-mutant tumors, as shown by unprecedented response rates of $25 \%$ in patients with $B R A F$-mutant tumors. ${ }^{31}$ Evidence from nivolumab and pembrolizumab studies indicates that these agents have efficacy in mCRC with MSI-H activity/dMMR.

To further enhance the possible therapeutic benefits of immunotherapy, the CheckMate 142 study also explored combination therapy with nivolumab and ipilimumab. Ipilimumab is an antibody targeting the CTLA-4 receptor; combination therapy with an anti-PD-1 antibody was shown to be more active than nivolumab monotherapy. Combination therapy appeared to provide a higher response rate $(55 \%)$ relative to that of the monotherapy group $(31 \%))^{36}$ At 12 months, nivolumab plus ipilimumab had longer survival (PFS, 71\%; OS, $85 \%$ ) than nivolumab alone (PFS, $50 \%$; OS, $73 \%){ }^{36}$

To investigate the therapeutic benefit of PD-1 immunotherapy in the firstline setting for patients with $\mathrm{dMMR}$ or MSI-H mCRC, the KEYNOTE-177 trial is currently ongoing (NCT02563002). The PFS data were presented at the American Society for Clinical Oncology 2020 Virtual Scientific Program, and there was a clear improvement with pembrolizumab with a doubling of median PFS over investigator's choice of chemotherapy (16.5 vs 8.2 months; HR, $0.60 ; 95 \%$ CI, $0.45-0.80 ; P=.0002)$ and only one-third of the grade 3-5 adverse event rate $(22 \%$ vs $66 \%) .{ }^{37}$ These findings have changed the standard of care to immunotherapy in the first-line setting for patients with dMMR or MSI-H mCRC and recently resulted in FDA approval. ${ }^{35}$ The COMMIT study is another randomized, open-label phase III study that compares mFOLFOX6/bevacizumab vs atezolizumab monotherapy vs mFOLFOX6/bevacizumab plus atezolizumab as first-line interventions for patients with $\mathrm{dMMR} / \mathrm{MSI}-\mathrm{H} \mathrm{mCRC}$ (NCT02997228).

\section{HER2 Amplification}

Gene amplification of HER2, also referred to as $E R B B 2$, is well described in breast and gastric cancers. Recently, HER2 amplification emerged as a therapeutic target in mCRC. HER2 overexpression is defined by immunohistochemistry (IHC) and amplification by fluorescence in situ hybridization (FISH). By IHC, colorectal tumor samples that show intense staining $(3+)$ in greater than $50 \%$ of cells are designated HER2-positive. By FISH, an ERB$\mathrm{B} 2$ :CEN17 ratio of 2.0 or less in $50 \%$ or more of cells (when counting ERBB2 and CEN17 signals from 100 nuclei per case) indicates HER2 amplification. ${ }^{38}$ Using this analytical methodology, the incidence of HER2/neu amplification in mCRCs overall is low (approximately 5\%). However, when analyzing mCRCs with a known rectal primary, the incidence can range from $12.4 \%$ to $26.7 \%$. $^{38-40}$

The open-label phase 2 HERACLES trial enrolled 27 patients with KRAS exon 2 WT, HER2-positive MCRC who were treated with the HER2 monoclonal antibody trastuzumab and the dual HER2/EGFR TKI lapatinib. ${ }^{41}$ Results revealed an ORR of $30 \%$, median PFS of 21 weeks (95\% CI, 16-32), median OS of 46 weeks (95\% CI, 33-68), and manage- able toxicities. ${ }^{41}$ In a follow-up analysis, the plasma copy number of ERBB2 amplifications was assessed in 48 plasma samples from 29 of the HERACLES trial's HER2-positive patients. ${ }^{42}$ The MyPathway phase 2 basket trial enrolled a subset of patients with treatment-refractory HER2-positive mCRC $(\mathrm{N}=57)$ and treated them with dual HER2-targeted therapy, trastuzumab and pertuzumab. ${ }^{43}$ The treatment regimen was generally well tolerated and the response rate was $32 \% .43$ Ongoing clinical trials are further investigating HER2-targeted therapies vs standard chemotherapy (NCT03365882, NCT03043313). The novel antibodydrug conjugate trastuzumab deruxtecan showed a promising $45.3 \%$ response rate in a heavily pretreated patient population with HER2-positive mCRC. ${ }^{44}$

\section{NTRK Fusions}

NTRK gene fusions, which encode TRKs, are rare but highly actionable. NTRK fusions lead to oncogenic overexpression of chimeric oncoproteins, which independently activate downstream signaling. ${ }^{45}$ One multiphase trial explored the ORRs of TRK fusion-positive cancer patients $(\mathrm{N}=55)$ to larotrectinib, a highly selective TRK inhibitor. ${ }^{46}$ Seven percent $(\mathrm{N}=4)$ of the patients treated had CRC, and their ORR was 75\% (95\% CI, 61\%$85 \%$ ), with durable responses seen at 1 year. ${ }^{46}$ The results from two phase 1 trials, ALKA-372-001 and STARTRK-1, which tested the safety and efficacy of entrectinib, a multitargeted pan-TRK, ROS, and ALK inhibitor, in the treatment of TKI treatment-naïve patients with advanced or metastatic solid tumors, were reported together; they revealed that entrectinib was well tolerated and led to durable clinical responses lasting as long as 2 years. ${ }^{47}$ Of the 119 patients treated, $15 \%$ had gastrointestinal primary tumors $(\mathrm{N}=18)$. In the phase 2 portion of these studies, 1 patient with $\mathrm{mCRC}$ and an NTRK fusion had disease response lasting 2.6 months, which demonstrated Continued on page 360 


\section{Par \\ (क) \\ (in) \\ W \\ e began the new decade with the satisfying news \\ of a palpable, praisewor-}

thy decrease in cancer mortality in the United States. This success is a result of continued research and investment in the understanding of cancer biology, and it is reflective of improved screening, improvements in adjuvant therapy, and maybe most impactful, the dramatic breakthroughs that immuno-oncology has brought. These results could not be obtained without decades of hard work within our highly collaborative environment composed of academic researchers, industry innovation and investment, supportive regulatory policies, and the "all-in" engagement of our patient partners.

Although I always held out hope, I never thought I would see such dramatic advances in my lifetime. Significant progress has been made in many of our most recalcitrant cancers, including lung cancer, kidney cancer, melanoma, hematological malignancies, and many others. But as we celebrate these achievements, we must remember that the successes we have achieved do not reliably equate with cures, which are our patients' fundamental expectation and our true goal. We must remember that we continue to practice "in the dark," unsure of who should get which drugs, and who might not need any drugs, radiation, or even surgery. We practice trial-and-error medicine, full of waste and residual toxicity. Cancer care is far from optimized, and much work remains ahead.

The first year of a new decade is a perfect time to reflect on the bigger picture of cancer care around the world. While we in the United States and other richer regions of the world have enjoyed many medical successes, most of our brothers and sisters coinhabiting our planet have not. Cancer care has become a luxury item, available only to those with either significant personal wealth or the good fortune to live in countries with strong economies. The difference between "the haves" and "the havenots" is striking, and in no medical discipline is this more dramatic than in oncology.

Let's face it-cancer care is complicated: It requires a large, highly trained, multidisciplinary team for it to be optimally delivered. The best teams must include experienced, specialized surgeons; medical oncologists; radiation oncologists; all of the affiliated professionals who assist in the diagnosis and management of patients, such as gastroenterologists, pulmonologists, and interventional radiologists; and, of course, nurses, nutritionists, and a building full of pieces of highly complex equipment, with skilled individuals who know how to run them.

Most of the world's population faces a very different level of health care. They struggle to have clean water and clean air, and to have basic health needs met. Such people are forced to make hard choices, or have had the will and foresight to make strategic changes that we, in the United States, have not yet been bold enough to make. Here, unanswered questions include: Is health care a right or privilege? Should everyone have access to unlimited care? What is the value of medical services? If you had to buy these services yourself, with your own money, would you?

We know that the health care system in the United States is not sustainable for much longer, and cancer care-specifically, the widespread use of immunotherapy-is accelerating the oncoming economic crisis. Other nations have made the tough choices, in some cases limiting access to newer, expensive therapies that do not meet economically derived value metrics. How will we respond as a nation when we face the same social and economic decision points?

At a time when nations are building walls, we in the cancer community must deepen our commitments to each other through collaborative research and data sharing. Our only way forward is to work together, especially as we expand our understanding and the ability to measure the complexities of cancers. The next great frontier for cancer research is precision medicine; with it comes the expanded incorporation of complex molecular profiling, linked to artificial intelligence-based machine learning, into our therapies and decision-making.

While recognizing that basic molecular testing of tumors is part of everyday practice, we are just beginning to understand this rapidly evolving field. If we reflect back, even on the past decade, we have evolved from a culture where no molecular testing was done for most cancers, to a time when single or "oligo" testing was performed, to today, when broad molecular profiling is rapidly becoming the standard of care. In less than a decade, genetic sequencing has moved from being a research question with no insurance coverage, to garnering FDA approvals, to becoming accepted by insurance companies as the standard of care.

As our tests have become increas- 
ingly complicated, we have come to recognize the importance of tissue quality and of preanalytic management of the specimen. We see more clearly the need to understand the actual assays that are being run, and to appreciate how one type of assay compares with another-eg, tissue vs liquid samples. Also, as with any new technology, we have seen a widening knowledge gap among us clinicians. As the technology and complexity of precision medicine have changed, we have fallen behind in our ability to interpret our patients' results. Some clinicians have become so frustrated and confused that they have stopped ordering molecular testing altogether. Others choose to order tests that yield a relatively low percentage of results that dramatically alter treatment decisions. Still others misinterpret results, causing additional medical expense and patient anxiety. And then, in many parts of the world, clinicians simply do not want to know the results of any tests they could theoretically order because, while they know that those results could indicate applicability of the latest promising treatment (eg, checkpoint inhibitors for microsatellite instability-high tumors), they do not have access to these therapies, leaving them only with regret over what might have been.

The rising cost of drug development is typically the first reason put forward to explain the rising cost of drugs, once they are approved. No doubt, drug development has become very expensive, and while the issue is multifactorial, a major reason is the rise of precision medicine. As we recognize that not all cancers are alike and subdivide them into smaller and smaller subgroups, trials become increasingly expensive. It costs more to find the patients, it takes longer to accrue the studies, and then, in the end, there is a smaller market of patients to purchase the therapy, limiting the return on investment.
At least within the United States, our government regulatory bodies have been keeping step with the rapid change in drug development and cancer technology. No longer is the only gold standard for drug approval a series of large phase 3 randomized trials. Don't get me wrong; this is still a standard route for drug discovery and approval. However, as we have increasingly identified molecular targets in patients with fatal cancers that portend a very high benefit rate $(<50 \%)$ with improved safety signals, our regulatory agencies have granted rapid approvals. So instead of "slogging" through large randomized trials in the hope of a hazard ratio of .8 , trials are now being designed with target hazard ratios of .5 and lower, results that don't actually require a statistician to confirm positivity. Given that our science is there, and our regulatory bodies are behind us, we need to play by new rules.

For the past several years, I have been fortunate to help build and lead a US-based collaborative group of investigators charged with studying the science of precision medicine, the Precision Oncology Alliance. It was only through coordinating our efforts and sharing our vast amounts of molecular and clinical data that we were able to uncover the many discoveries hidden within. Collectively, we understand the value in numbers, of the ability to characterize our patients into new subgroups, and of beginning to apply the latest therapies using prospective enrichment. As we move forward, increasingly complex molecular results are being analyzed using artificial intelligence/machine learning to provide us with more prognostic and predictive insights.

We are beginning to embrace tumor heterogeneity, and we continue to invest more in the practice of biomarker discovery. This work will uncover more targets and allow for better therapeutic decisions, meaning that we can all ultimately fight a smarter, more efficient war against cancer. Critical to our success will be global outreach and expansion of cancer care markets. We have well-established science showing us that cancers vary by global distribution, maybe secondary to differences in the microbiome. This suggests that results from trials performed in one region of the world may not be applicable in other regions.

As part of our current work, representatives from around the world are building a global precision network, the Oncology Alliance for Individualized Medicine (Onco Al-Med), that links cancer centers in 4 continents. By analyzing tumors optimized for tissue quality-limiting ischemia timeand performing extensive testing that includes whole genome sequencing, whole RNA transcriptome, proteomics, and ultimately metabolomics on both the tumor and normal tissues, coupled with artificial intelligence learning, we expect to push the field of precision medicine to its next phase. We also hope that by having large populations of patients "profiled" prospectively, we will lower the cost of drug development through rapid patient identification.

Precision medicine, while still in its infancy, is accepted and here to stay. Rapid improvements in technology continue, which for all their benefits also create a widening knowledge gap, which we must close. To accomplish this, we must partner on a global scale, both because the science requires this and, more importantly, because we are morally compelled to share our success with our fellow global citizens. Cancer care can no longer be a luxury item, only for the rich. We must work toward wide access. Opening markets through collaborative science will secure the return on investment for our business partners, and secure our progress toward finding the cures for cancer.

Marshall is director, Otto J. Ruesch Center for the Cure of Gastrointestinal Cancers and chief, Division of Hematology and Oncology, Lombardi Comprehensive Cancer Center, MedStar Georgetown University Hospital. 


\section{MEET OUR EXPERT}

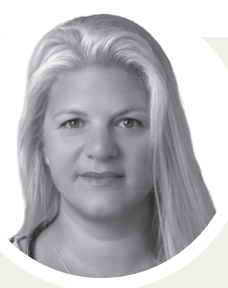

Traina is a medical oncologist at Memorial Sloan Kettering Cancer Center.

\section{The Path to Improving Breast Cancer Outcomes}

\section{Targeting the androgen receptor pathway in triple-negative breast cancer}

With the rise of genetic sequencing and rapidly advancing targeted therapies, outcomes for patients with breast cancer are improving. As the understanding of driver genes and clinical pathways continues to increase, newer treatments for patients with various specific forms of breast cancer are beginning to emerge.

ONCOLOGY ${ }^{\circledR}$ recently sat down with Tiffany A. Traina, $\mathrm{MD}$, a medical oncologist at Memorial Sloan Kettering (MSK) Cancer Center, to discuss recent developments in breast cancer care, the upcoming clinical trials that may change the standard of care, and her current work in targeting the androgen receptor (AR) pathway for patients with triple-negative breast cancer (TNBC).

\section{(1. What are the most exciting developments going on in breast cancer treatment?}

TRAINA: A few really exciting areas [emerged] in the management of breast cancer over the past year. The first is in the space of treating women with HER2-positive advanced breast cancer. Two new FDA approved agents in that space have really impressive efficacy results. One is a new antibody-drug conjugate, trastuzumab deruxtecan
(Enhertu), and the other is an oral tyrosine kinase inhibitor, tucatinib. Tucatinib has shown really promising survival data in patients with brain metastases, which is a tremendous area of unmet need. These 2 powerful new agents will help improve outcomes for our women with advanced HER2-positive breast cancer.

Another area of great interest with new exciting data is in the TNBC space, where we've seen FDA approval of a new antibody-drug conjugate called sacituzumab govitecan (Trodelvy). This has really offered hope and promise for patients being treated in the third line or later for TNBC, which is another area of tremendous unmet need, and it really gives us more options for our patients. Additionally, we're seeing perhaps an expanded role of immunotherapy in TNBC, both in the early-stage setting and in first-line metastatic disease. So, there are a lot of new data to be aware of and also discussions about how to incorporate these new drugs into our practice.

1. You've done a fair targeting the AR, even in TNBC. Why is that approach working, and which subset of TNBC patients is it working for?
TRAINA: That's a great question. I think what we've all come to recognize is that TNBC is quite heterogeneous, and it really is a composite term for tumors that are estrogen-negative, progesterone-negative, and HER2-negative, but they have a constellation of other drivers. Multiple research groups-including ours at MSK-have described a subset of these triple-negative cancers that are driven by an endocrine signature. And it seems to be the presence of the AR, as well as signaling through that AR pathway, that has had compelling evidence of potential benefit from targeting that pathway. So, we've led multiple single-arm phase 2 studies of various androgen-targeted oral therapies that are really well tolerated in this specific subset of patients with TNBC. And I'm happy to say that we actually have secured funding and grant support to conduct a randomized trial of the AR antagonist enzalutamide (Xtandi) against treatment of physician's choice. That particular trial, which will be really rich with correlative studies, will be conducted through the Translational Breast Cancer Research Consortium, and it should be available at sites around the country.

The idea is that for a tumor that's very endocrine-driven, very hormone-driven, actually 
targeting that pathway instead of treating with chemotherapy may offer a really well-tolerated approach that could be of benefit. So, rather than adding [a] chemotherapy agent, the idea is that you could lead in with an endocrine approach, and then at some point introduce chemotherapy. It's not unlike the way we manage an estrogen-driven cancer, using estrogen receptor (ER)-targeted therapies and then ultimately moving those patients to chemotherapy. The design of this clinical trial is such that it could answer that question: Is there a clear subgroup of patients with TNBC who could benefit from a nonchemotherapy approach?

Q: The use of enzalutamide, and similar agents, is typically associated with treating prostate cancer. Will all these agents be considered to treat breast cancer?

TRAINA: Well, we've tried several already. There has been published work using the older and still-available drug bicalutamide (Casodex), as well as work using investigational agents, not yet approved for prostate cancer, that do target the androgen-signaling pathway. Enzalutamide has probably been the one furthest developed, with published mature phase 1 and phase 2 data in breast cancer. So it was natural for that to be the leading agent to leap to a randomized trial right now. Of course, what's being developed in prostate cancer could also offer promise for our patients with AR-driven breast cancer.

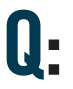

Is targeting the AR pathway a recent development, or is this something that was always

\section{known but has only recently come to the forefront?}

TRAINA: The published data on a potential role for targeting the AR pathway really date back more than a decade. However, the relevance of that role is something that's been more recently clarified. I think we're still trying to understand the best biomarker to be able to predict for benefit. It may be something beyond just the presence of the receptor, but perhaps more of a composite marker to predict benefit. A lot remains to be understood. Some data show that the AR may be relevant not just in TNBC, but also in ER-positive breast cancer where the coexpression of ER and AR is quite high. In HER2-positive breast cancer, there is also a higher rate of coexpression of the AR and HER2, so we're still trying to figure out a lot.

\section{You mentioned new applications for immunotherapies in TNBC. What is driving the ability to expand the use of immunotherapies?}

TRAINA: We've learned a few things that continue to be clarified around immunotherapy in TNBC. One is learning in which line of therapy these drugs will offer the greatest benefit. We've come to see that for metastatic disease, introducing these agents early in the first-line setting shows greater promise than using them in a later line. We are also now seeing, from early-stage trials, that moving these checkpoint inhibitors up into the neoadjuvant setting, even potentially the adjuvant setting, has great benefit. Another piece that we've clarified is that as a single agent, each of these drugs has not appeared to be of substantial efficacy, but when they're partnered with other agents, whether those are cytotoxic chemotherapy or radiation therapy, that's where we really start to see a big impact from their mechanism of action.

So, over time we're clarifying [3 questions]: In what line of therapy do you use these drugs? What partners should you use with these drugs? And finally, very important, [can we] refine the predictive biomarker of benefit? The last is still an area that's a little bit muddy; there are several different definitions for PD-L1 positivity and different companion assays are available, depending on which checkpoint inhibitor you're talking about. Yet I think we're seeing greater benefit as we're better able to define the population who can respond to these agents, and we're getting better at refining which patients are most likely to benefit [from them].

\section{What trial results are forward to seeing?}

TRAINA: We haven't yet touched on the ER-positive space. We've seen the press release [describing] the data for the PALLAS trial not meeting its primary end point to show an advantage of palbociclib (Ibrance) in combination of endocrine therapy in the adjuvant setting, and we've seen a press release [showing that] abemaciclib (Verzenio) potentially has benefit in the adjuvant setting. Seeing these data in greater detail, to understand how [these agents can] beneficially impact early-stage, ER-positive breast cancer, is something I'm really looking forward to.
Another really important study will include the phase 3 results of trastuzumab deruxtecan in HER2-positive breast cancer. This antibody-drug conjugate had expedited approval based on really amazing efficacy in a single-arm phase 2 study in HER2-positive disease. So, it will be fantastic to see those phase 3 data-likely confirmatory-and also, perhaps, to see some brain data from that agent in HER2-positive breast cancer.

In TNBC, a lot remains to be understood, so [I'm looking forward to] seeing adjuvant data around incorporation of checkpoint inhibitors. Many trials thus far have been neoadjuvant trials with pathologic complete response as the primary end point, but what's ultimately really important is the survival impact of using these drugs. So those would be some of the highlights I'm really looking forward to that would be practice-changing.

\section{O. Of the studies 1. in which you've participated, which make you most proud?}

TRAINA: I did all of my oncology training at MSK and so being a part of dose density — establishing dose density as a backbone chemotherapy administration schedule-is exciting. I would also say that the entire AR program within TNBC is something I'm really proud of, because that started with an observation by one of our pathologists and really translated into multiple phase 1 studies for women with breast cancer to prove the safety of various agents from [the realm of] prostate cancer. Those moved into phase 2 programs and now I'm really happy to see that [evolve] into a randomized trial. 
That's been exciting work to be a part of: seeing the entire drug development, the more than a decade of work to get from observation in the lab at MSK all the way through to a randomized trial. Plus, [I'm proud of being part of] the work in the HER2-positive space and part of the work with trastuzumab deruxtecan. At MSK, we've had a part in developing all of the HER2-targeted therapies, really, and it's so rewarding to get to see highly effective drugs [in the end] and bring them to our patients.

\section{With the coronavirus disease 2019 (COVID-19) pandemic,}

\section{cancer care and cancer screenings were down. Are we in the recovery phase yet?}

TRAINA: I think we have seen a recovery in volume and clinical volume. But I think what we have recognized, and data are now published to support this, is that in the beginning of the throes of the pandemic, [especially] in March and April, patients were fearful of coming out of their homes, and [especially of] coming into a clinical environment or into congested cities, [or both]. [This was true for] receiving care, and for screening studies, in which the risk-benefit balance in patients' eyes might have favored avoiding COVID-19 and putting off screening and surveillance studies. A body of literature is developing that suggests we [should be concerned that] we'll perhaps see a wave of cancer diagnoses at a [later point in time] because of the COVID-19-related delays in diagnosis.

I think we are still very much in the first-wave experience of this pandemic, and [unfortunately] cancer doesn't just wait. We are now about 5 months into this experience, at least, and in terms of care- whether that means nonelective cancer operations, chemotherapy, immunotherapy, targeted therapy, endocrine therapies - these need to go on, with the care of our patients remaining a priority. We are all finding ways to continue to deliver high-quality care in a safe environment that takes into account social distancing, appropriate personal protective equipment, and psychosocial support, as well as visitor restriction policies and family safety. It really has added layers of complexity to delivering our care, but it's necessary.

\section{FINANCIAL DISCLOSURE: The}

authors have no significant financial interest in or other relationship with the manufacturer of any product or provider of any service mentioned in this article.

\section{Review Article: GI Cancer}

Continued from page 357

a potential benefit to targeting TRK in mCRC. Acquired TRK resistance mechanisms known as solvent front mutations can develop. Trials exploring next-generation TRK inhibitors such as LOXO-195/ selitrectinib to overcome these resistance mechanisms are ongoing. ${ }^{48}$

\section{Conclusions}

mCRC remains a leading cause of cancer death in the United States, but research is beginning to show that alteration of the course of this disease may be possible with innovative interventions. Chemotherapy has provided reliable initial treatment, and molecular profiling is creating new therapeutic options that give patients hope of increased survival. The research community has demonstrated the need to profile patients with mCRC for RAS and BRAF mutations, MSI-H status, HER2 amplifications, and NTRK fusions. Knowledge of primary tumor location along with specific targetable tumor mutations is beginning to change the outlook for patients with mCRC.

\section{FINANCIAL DISCLOSURE:}

The authors have no significant financial interest in or other relationship with the manufacturer of any product or provider of any service mentioned in this article.

ACKNOWLEDGMENTS: We would like to thank Marion L. Hartley, $\mathrm{PhD}$, for her edits to this review.

\section{KEY REFERENCES}

1. Cancer facts \& figures 2020.

American Cancer Society.

Accessed August 10, 2020.

https://www.cancer.org/research/ cancer-facts-statistics/all-cancerfacts-figures/cancer-factsfigures-2020.html

2. Surveillance, Epidemiology, and End Results (SEER) Program. National Cancer Institute. Accessed August 19, 2020. https://www.seer.cancer.gov

3. Bettington M, Walker N,
Clouston A, et al. The serrated pathway to colorectal carcinoma: current concepts and challenges. Histopathology. 2013;62(3):367386. doi:10.1111/his.12055

4. Stintzing S, Tejpar S, Gibbs $\mathrm{P}$, et al. Understanding the role of primary tumour localisation in colorectal cancer treatment and outcomes. Eur J Cancer. 2017;84:69-80. doi:10.1016/j. ejca.2017.07.016

5. Brulé SY, Jonker DJ, Karapetis CS, et al. Location of colon cancer (right-sided versus leftsided) as a prognostic factor and a predictor of benefit from cetuximab in NCIC CO.17. Eur J Cancer. 2015;51(11):1405-1414. doi:10.1016/j.ejca.2015.03.015

For full reference list, visit cancernetwork.com/ CRCprofiling

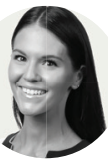

\section{Armstrong}

is a third-year medical hematology/ oncology fellow at Georgetown Lombardi Comprehensive Cancer Center.

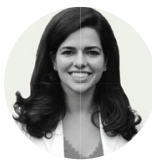

\section{Malley}

is a third-year medical student at the Georgetown University School of Medicine

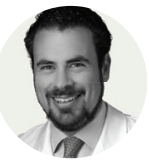

\section{Weinberg}

is an assistant professor of medicine, Ruesch Center for the Cure of Gastrointestinal Cancers, Lombardi Comprehensive Cancer Center, Georgetown University Medical Center. 
IN THE TREATMENT OF RELAPSED REFRACTORY MULTIPLE MYELOMA IN COMBINATION WITH POMALIDOMIDE AND DEXAMETHASONE (Pd)

\section{ACHIEVE GREATER OUTCOMES FOR YOUR PATIENTS}

SARCLISA is an anti-CD38 therapy proven to deliver superior PFS (median PFS of 11.53 months with SARCLISA + Pd vs 6.47 months with Pd alone, $\mathrm{HR}=0.596,95 \% \mathrm{Cl}: 0.44,0.81, P=0.0010$ ). SARCLISA also demonstrated a significant increase in ORR $(60.4 \%$ with SARCLISA + Pd [95\% Cl: $52.2 \%, 68.2 \%]$ vs $35.3 \%$ with Pd alone [95\% Cl: $27.8 \%, 43.4 \%], P<0.0001)^{1 *}$
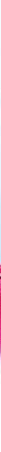

\section{NCCN Clinical Practice Guidelines in Oncology (NCCN Guidelines ${ }^{\circledR}$ ) Category 1 recommendation for isatuximab-irfc (SARCLISA)}

Isatuximab-irfc (SARCLISA) is the only $\mathrm{mAb}$ in combination with pomalidomide and dexamethasone included as an NCCN Guidelines ${ }^{\circledR}$ Category 1 recommended regimen for previously treated multiple myeloma. ${ }^{2}$

NCCN makes no warranties of any kind whatsoever regarding their content, use or application and disclaims any responsibility for their application or use in any way.

*ORR included SCR, CR, VGPR, and PR. SCR, CR, VGPR, and PR were evaluated by an IRC using the IMWG response criteria.'

$C R=$ complete response; IMWG=International Myeloma Working Group; IRC=independent response committee; $m A b=$ monoclonal antibody; NCCN=National Comprehensive Cancer Network; ORR=overall response rate; PFS=progression-free survival; PR=partial response; $\mathrm{SCR}=$ stringent complete response; $\mathrm{VGPR}=$ very good partial response.

\section{Indication}

SARCLISA (isatuximab-irfc) is indicated, in combination with pomalidomide and dexamethasone, for the treatment of adult patients with multiple myeloma who have received at least two prior therapies including lenalidomide and a proteasome inhibitor.

\section{Important Safety Information}

\section{CONTRAINDICATIONS}

SARCLISA is contraindicated in patients with severe hypersensitivity to isatuximab-irfc or to any of its excipients.

\section{WARNINGS AND PRECAUTIONS}

\section{Infusion-Related Reactions}

Infusion-related reactions (IRRs) have been observed in $39 \%$ of patients treated with SARCLISA. All IRRs started during the first SARCLISA infusion and resolved on the same day in $98 \%$ of the cases. The most common symptoms of an IRR included dyspnea, cough, chills, and nausea. The most common severe signs and symptoms included hypertension and dyspnea.

Please see Important Safety Information throughout, and accompanying brief summary of full Prescribing Information.

SARCLISA (isatuximab-irfc) Injection for IV use $\mid 500 \mathrm{mg} / 25 \mathrm{~mL}, 100 \mathrm{mg} / 5 \mathrm{~mL}$ 


\title{
Choose SARCLISA + Pd to Offer Improved Outcomes to More Patients vs Pd Alone
}

\author{
Studied in the phase 3 ICARIA-MM trial, which included \\ patients with poor prognostic factors ${ }^{1}$
}

\section{Based on the ICARIA-MM trial, SARCLISA + Pd is a treatment choice for patients with relapsed refractory multiple myeloma}

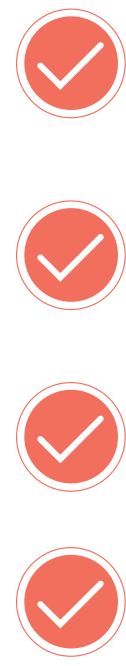

\author{
Who have received at least 2 prior therapies, \\ including lenalidomide and a PI
}

Who may have renal impairment (creatinine clearance $<60 \mathrm{~mL} / \mathrm{min} / 1.73 \mathrm{~m}^{2}$ ), high cytogenetic risk, or a history of COPD or asthma

\section{Who may have poor performance status or are $\geq 75$ years of age}
Who are refractory to lenalidomide, a PI, or both

STUDY DESIGN: ICARIA-MM (NCTO2990338), a multicenter, open-label, randomized, phase 3 study, evaluated the efficacy and safety of SARCLISA in 307 patients with relapsed refractory multiple myeloma who had received at least 2 prior therapies, including lenalidomide and a PI. Patients received either SARCLISA $10 \mathrm{mg} / \mathrm{kg}$ administered as an IV infusion in combination with Pd ( $\mathrm{n}=154)$ or Pd alone ( $\mathrm{n}=153)$, administered in 28-day cycles until disease progression or unacceptable toxicity. SARCLISA was given weekly in the first cycle and every 2 weeks thereafter. Pomalidomide $4 \mathrm{mg}$ was taken orally once daily from day 1 to day 21 of each 28-day cycle. Low-dose dexamethasone (orally or IV) $40 \mathrm{mg}$ ( $20 \mathrm{mg}$ for patients $\geq 75$ years of age) was given on days 1, 8, 15, and 22 for each 28-day cycle. PFS was the primary endpoint; ORR and OS were key secondary endpoints. PFS results were assessed by an IRC, based on central laboratory data for M-protein, and central radiologic imaging review using the IMWG criteria. Median follow-up was 11.6 months.

PATIENT CHARACTERISTICS: The median patient age was 67 years (range, 36 to 86 ), and $20 \%$ of patients were $\geq 75$ years of age. Ten percent of patients entered the study with a history of COPD or asthma. The proportion of patients with renal impairment (creatinine clearance $<60 \mathrm{~mL} / \mathrm{min} / 1.73 \mathrm{~m}^{2}$ ) was $34 \%$. The ISS stage at study entry was I in $37 \%$, II in $36 \%$, and III in $25 \%$ of patients. Overall, $20 \%$ of patients had high-risk chromosomal abnormalities at study entry: del(17p), $t(4 ; 14)$, and $t(14 ; 16)$ were present in $12 \%, 8 \%$, and $2 \%$ of patients, respectively. The median number of prior lines of therapy was 3 (range, 2 to 11). All patients received a prior PI, all patients received prior lenalidomide, and $56 \%$ of patients received prior stem cell transplantation; the majority of patients (93\%) were refractory to lenalidomide, $76 \%$ to a $\mathrm{PI}$, and $73 \%$ to both an immunomodulator and a PI.'

\section{Important Safety Information (cont'd)}

\section{Infusion-Related Reactions (cont'd)}

To decrease the risk and severity of IRRs, premedicate patients prior to SARCLISA infusion with acetaminophen, $\mathrm{H}_{2}$ antagonists, diphenhydramine or equivalent, and dexamethasone. Monitor vital signs frequently during the entire SARCLISA infusion. For patients with grade 1 or 2 reactions, interrupt SARCLISA infusion and provide appropriate medical support. If symptoms improve, restart SARCLISA infusion at half of the initial rate, with supportive care as needed, and closely monitor patients. If symptoms do not recur after 30 minutes, the infusion rate may be increased to the initial rate, and then increased incrementally. In case symptoms do not improve or recur after interruption, permanently discontinue SARCLISA and institute appropriate management. Permanently discontinue SARCLISA if a grade 3 or higher IRR occurs and institute appropriate emergency medical management. 


\section{SARCLISA + Pd Extended Median PFS to 1 Year}

\section{Superior PFS with SARCLISA + Pd vs Pd alone'}

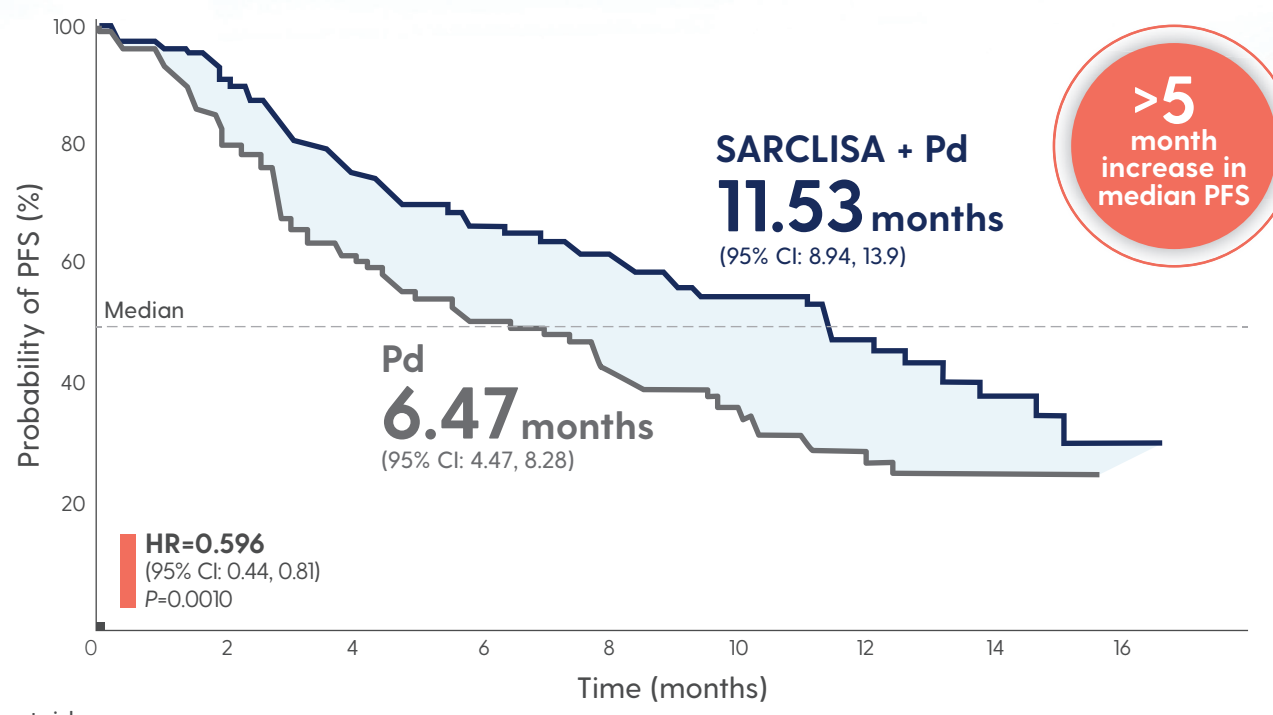

Patients at risk

SARCLISA + Pd 154

Pd 153

$\begin{array}{ll}129 & 106 \\ 105 & 80\end{array}$

89
63

81

52

30
17

14

The median duration of treatment was 41 weeks with SARCLISA + Pd vs 24 weeks with Pd. ${ }^{1}$

At a median follow-up time of 11.6 months, 43 patients (27.9\%) receiving SARCLISA + Pd and 56 patients $(36.6 \%)$ receiving Pd had died. Median OS was not reached for either treatment group at interim analysis. The OS results at interim analysis did not reach statistical significance.'

\section{SARCLISA + Pd showed a significant increase in ORR ${ }^{1 *}$}

\begin{tabular}{|c|c|c|}
\hline SARCLISA + Pd & 154) & $\mathbf{P d}(\mathrm{n}=153)$ \\
\hline $60.4 \%$ ORR & $P<0.0001$ & $35.3 \%$ ORR \\
\hline $31.8 \% \geq V G P R$ & $\sim 4 \times$ increase & $\mathbf{8 . 5} \% \geq V G P R$ \\
\hline 35 days & $\begin{array}{l}\text { Median time to first response } \\
\text { among responders }\end{array}$ & 58 days \\
\hline
\end{tabular}

*ORR included sCR, CR, VGPR, and PR. ORR: SARCLISA + Pd (95\% Cl: 52.2\%, 68.2\%), Pd (95\% Cl: $27.8 \%, 43.4 \%)$

\section{Important Safety Information (cont'd)}

\section{Neutropenia}

SARCLISA may cause neutropenia. Neutropenia (reported as laboratory abnormality) occurred in $96 \%$ of patients and grade 3-4 neutropenia occurred in $85 \%$ of patients treated with SARCLISA, pomalidomide, and dexamethasone (Isa-Pd). Febrile neutropenia occurred in $12 \%$ of patients and neutropenic infections, defined as infection with concurrent grade $\geq 3$ neutropenia, occurred in $25 \%$ of patients treated with Isa-Pd. The most frequent neutropenic infections included those of upper respiratory tract $(10 \%)$, lower respiratory tract $(9 \%)$, and urinary tract $(3 \%)$.

Please see Important Safety Information throughout, and accompanying brief summary of full Prescribing Information. 


\section{Important Safety Information (cont'd)}

\section{Neutropenia (cont'd)}

Monitor complete blood cell counts periodically during treatment. Consider the use of antibiotics and antiviral prophylaxis during treatment. Monitor patients with neutropenia for signs of infection. In case of grade 4 neutropenia, delay SARCLISA dose until neutrophil count recovery to at least $1.0 \times 10^{\circ} / \mathrm{L}$, and provide supportive care with growth factors, according to institutional guidelines. No dose reductions of SARCLISA are recommended.

\section{Second Primary Malignancies}

Second primary malignancies were reported in 3.9\% of patients in the SARCLISA, pomalidomide, and dexamethasone (Isa-Pd) arm and in $0.7 \%$ of patients in the pomalidomide and dexamethasone (Pd) arm, and consisted of skin squamous cell carcinoma ( $2.6 \%$ of patients in the Isa-Pd arm and in $0.7 \%$ of patients in the Pd arm), breast angiosarcoma (0.7\% of patients in the lsa-Pd arm), and myelodysplastic syndrome $(0.7 \%$ of patients in the Isa-Pd arm). With the exception of the patient with myelodysplastic syndrome, patients were able to continue SARCLISA treatment. Monitor patients for the development of second primary malignancies.

\section{Laboratory Test Interference}

Interference with Serological Testing (Indirect Antiglobulin Test)

SARCLISA binds to CD38 on red blood cells (RBCs) and may result in a false positive indirect antiglobulin test (indirect Coombs test). In ICARIA-multiple myeloma (MM), the indirect antiglobulin test was positive during SARCLISA treatment in $67.7 \%$ of the tested patients. In patients with a positive indirect antiglobulin test, blood transfusions were administered without evidence of hemolysis. $A B O / R h D$ typing was not affected by SARCLISA treatment. Before the first SARCLISA infusion, conduct blood type and screen tests on SARCLISA-treated patients. Consider phenotyping prior to starting SARCLISA treatment. If treatment with SARCLISA has already started, inform the blood bank that the patient is receiving SARCLISA and SARCLISA interference with blood compatibility testing can be resolved using dithiothreitol-treated RBCs. If an emergency transfusion is required, non-cross-matched $A B O / R h D-$ compatible RBCs can be given as per local blood bank practices.

\section{Interference with Serum Protein Electrophoresis and Immunofixation Tests}

SARCLISA is an IgG kappa monoclonal antibody that can be incidentally detected on both serum protein electrophoresis and immunofixation assays used for the clinical monitoring of endogenous M-protein. This interference can impact the accuracy of the determination of complete response in some patients with lgG kappa myeloma protein.

\section{Embryo-Fetal Toxicity}

Based on the mechanism of action, SARCLISA can cause fetal harm when administered to a pregnant woman. SARCLISA may cause fetal immune cell depletion and decreased bone density. Advise pregnant women of the potential risk to a fetus. Advise females with reproductive potential to use an effective method of contraception during treatment with SARCLISA and for at least 5 months after the last dose. The combination of SARCLISA with pomalidomide is contraindicated in pregnant women because pomalidomide may cause birth defects and death of the unborn child. Refer to the pomalidomide prescribing information on use during pregnancy.

\section{ADVERSE REACTIONS}

The most common adverse reactions ( $\geq 20 \%$ ) were neutropenia (laboratory abnormality, $96 \%$ Isa-Pd vs $92 \% \mathrm{Pd}$ ), infusion-related reactions ( $38 \%$ Isa-Pd vs $0 \% \mathrm{Pd}$ ), pneumonia ( $31 \% \mathrm{Isa}-\mathrm{Pd}$ vs $23 \% \mathrm{Pd}$ ), upper respiratory tract infection (57\% Isa-Pd vs $42 \% \mathrm{Pd}$ ), and diarrhea (26\% with Isa-Pd vs $19 \% \mathrm{Pd}$ ). Serious adverse reactions occurred in $62 \%$ of patients receiving SARCLISA. Serious adverse reactions in $>5 \%$ of patients who received Isa-Pd included pneumonia (26\%), upper respiratory tract infections (7\%), and febrile neutropenia $(7 \%)$. Fatal adverse reactions occurred in $11 \%$ of patients (those that occurred in more than $1 \%$ of patients were pneumonia and other infections [3\%]).

\section{USE IN SPECIAL POPULATIONS}

Because of the potential for serious adverse reactions in the breastfed child from isatuximab-irfc administered in combination with Pd, advise lactating women not to breastfeed during treatment with SARCLISA.

\section{Please see accompanying brief summary of full Prescribing Information.}

References: 1. SARCLISA [prescribing information]. Bridgewater, NJ: sanofi-aventis U.S. LLC. 2. Referenced with permission from the NCCN Clinical Practice Guidelines in Oncology (NCCN Guidelines ${ }^{\circledR}$ ) for Multiple Myeloma V.4.2020. ๑ National Comprehensive Cancer Network, Inc. 2020. All rights reserved. Accessed June 16, 2020. To view the most recent and complete version of the guideline, go online to NCCN.org. 
SARCLISA ${ }^{\circledR}$ Rx Only

(isatuximab-irfc) injection, for intravenous use Brief Summary of Prescribing Information 1 INDICATIONS AND USAGE

SARCLISA is indicated, in combination with pomalidomide and dexamethasone, for the treatment of adult patients with multiple myeloma who have received at least two prior

therapies including lenalidomide and a proteasome inhibitor.

\section{DOSAGE AND ADMINISTRATION}

\subsection{Recommended Dosage}

- Administer pre-infusion medications [see Dosage and

Administration (2.2)].

- SARCLISA should be administered by a healthcare professional, with immediate access to emergency equipment and appropriate medical support to manage infusion-related reactions if they occur [see Warnings and Precautions (5.1)].

The recommended dose of SARCLISA is $10 \mathrm{mg} / \mathrm{kg}$ actual body weight administered as an intravenous infusion in combination with pomalidomide and dexamethasone, according to the schedule in Table 1 [see Clinical Studies (14) in the full prescribing information].

Table 1: SARCLISA Dosing Schedule in Combination with Pomalidomide and Dexamethasone

\begin{tabular}{|l|l|}
\hline Cycle & Dosing schedule \\
\hline Cycle 1 & Days 1, 8, 15, and 22 (weekly) \\
\hline Cycle 2 and beyond & Days 1, 15 (every 2 weeks) \\
\hline
\end{tabular}

Each treatment cycle consists of a 28-day period. Treatment is repeated until disease progression or unacceptable toxicity. SARCLISA is used in combination with pomalidomide and dexamethasone.

Missed SARCLISA Doses

If a planned dose of SARCLISA is missed, administer the dose as soon as possible and adjust the treatment schedule accordingly, maintaining the treatment interval.

\subsection{Recommended Premedications}

Administer the following premedications prior to SARCLISA infusion to reduce the risk and severity of infusion-related reactions [see Warnings and Precautions (5.1)]

- Dexamethasone $40 \mathrm{mg}$ orally or intravenously (or $20 \mathrm{mg}$ orally or intravenously for patients $\geq 75$ years of age).

- Acetaminophen 650 mg to 1000 mg orally (or equivalent).

- H2 antagonists.

- Diphenhydramine 25 mg to 50 mg orally or intravenously (or equivalent). The intravenous route is preferred for at least the first 4 infusions.

The above recommended dose of dexamethasone (orally or intravenously) corresponds to the total dose to be administered only once before infusion as part of the premedication and of the backbone treatment, before SARCLISA and pomalidomide administration.

Administer the recommended premedication agents 15 to 60 minutes prior to starting a SARCLISA infusion.

\subsection{Dose Modification}

No dose reduction of SARCLISA is recommended. Dose delay may be required to allow recovery of blood counts in the event of hematological toxicity [see Warnings and Precautions $(5.2,5.4)$ ]. For information concerning drugs given in combination with SARCLISA, see manufacturer's prescribing information. For other medicinal products that are administered with SARCLISA, refer to the respective current prescribing information.

\subsection{Preparation}

Prepare the solution for infusion using aseptic technique as follows: Calculate the dose $(\mathrm{mg})$ of required SARCLISA based on actual patient weight (measured prior to each cycle to have the administered dose adjusted accordingly) [see Dosage and Administration (2.1)]. More than one SARCLISA vial may be necessary to obtain the required dose for the patient.

- Parenteral drug products should be inspected visually for particulate matter and discoloration prior to administration, whenever solution and container permit.

- Remove the volume of diluent from the $250 \mathrm{~mL}$ Sodium Chloride Injection, USP, or 5\% Dextrose Injection,

USP diluent bag that is equal to the required volume of

SARCLISA injection.

- Withdraw the necessary volume of SARCLISA injection and dilute by adding to the infusion bag of $0.9 \%$ Sodium Chloride Injection, USP or 5\% Dextrose Injection, USP to achieve the appropriate SARCLISA concentration for infusion.

- The infusion bag must be made of polyolefins (PO) polyethylene $(P E)$, polypropylene (PP), polyvinyl chloride (PVC) with di-(2-ethylhexyl) phthalate (DEHP) or ethyl vinyl acetate (EVA)

- Gently homogenize the diluted solution by inverting the bag. Do not shake.

\subsection{Administration}

- Administer the infusion solution by intravenous infusion using an intravenous tubing infusion set (in PE, PVC with or without DEHP, polybutadiene [PBD], or polyurethane [PU]) with a 0.22 micron in-line filter (polyethersulfone [PES], polysulfone, or nylon).

- The infusion solution should be administered for a period of time that will depend on the infusion rate (see Table 2).

Use prepared SARCLISA infusion solution within 48 hours when stored refrigerated at $2^{\circ} \mathrm{C}-8^{\circ} \mathrm{C}$, followed by 8 hours (including the infusion time) at room temperature.

- Do not administer SARCLISA infusion solution concomitantly in the same intravenous line with other agents.

\section{Infusion Rates}

Following dilution, administer the SARCLISA infusion solution intravenously at the infusion rates presented in

Table 2. Incremental escalation of the infusion rate should be considered only in the absence of infusion-related reactions [see Warnings and Precautions (5.1) and Adverse Reactions (6.1)].

Table 2: Infusion Rates of SARCLISA Administration

\begin{tabular}{|l|c|c|c|c|c|}
\hline & $\begin{array}{c}\text { Dilution } \\
\text { Volume }\end{array}$ & $\begin{array}{c}\text { Initial } \\
\text { Rate }\end{array}$ & $\begin{array}{c}\text { Absence of } \\
\text { Infusion- } \\
\text { Related } \\
\text { Reaction }\end{array}$ & $\begin{array}{c}\text { Rate } \\
\text { Increment }\end{array}$ & $\begin{array}{c}\text { Maximum } \\
\text { Rate }\end{array}$ \\
\hline $\begin{array}{l}\text { First } \\
\text { infusion }\end{array}$ & $250 \mathrm{~mL}$ & $\begin{array}{c}25 \mathrm{~mL} \\
\text { hour }\end{array}$ & $\begin{array}{c}\text { For } 60 \\
\text { minutes }\end{array}$ & $\begin{array}{c}25 \mathrm{~mL} / \text { hour } \\
\text { every } 30 \\
\text { minutes }\end{array}$ & $\begin{array}{c}150 \mathrm{~mL} / \\
\text { hour }\end{array}$ \\
\hline $\begin{array}{l}\text { Second } \\
\text { infusion }\end{array}$ & $250 \mathrm{~mL}$ & $\begin{array}{c}50 \mathrm{~mL} / \\
\text { hour }\end{array}$ & $\begin{array}{c}\text { For } 30 \\
\text { minutes } \\
\text { hour for } 30 \\
\text { minutes then } \\
\text { increase by } \\
100 \mathrm{~mL} / \\
\text { hour every } 30 \\
\text { minutes }\end{array}$ & $\begin{array}{c}200 \mathrm{~mL} / \\
\text { hour }\end{array}$ & $\begin{array}{c}200 \mathrm{~mL} / \\
\text { hour }\end{array}$ \\
\hline $\begin{array}{l}\text { Subsequent } \\
\text { infusions }\end{array}$ & $250 \mathrm{~mL}$ & $\begin{array}{c}200 \\
\mathrm{~mL} / \\
\text { hour }\end{array}$ & - & - & \multicolumn{2}{|c|}{} \\
\hline
\end{tabular}

\section{CONTRAINDICATIONS}

SARCLISA is contraindicated in patients with severe hypersensitivity to isatuximab-irfc or to any of its excipients [see Warnings and Precautions (5.1)].

\section{WARNINGS AND PRECAUTIONS}

$\mathbf{5 . 1}$ Infusion-Related Reactions

Infusion-related reactions have been observed in 39\% of patients treated with SARCLISA /see Adverse Reactions (6.1)]. All infusion-related reactions started during the first SARCLISA infusion and resolved on the same day in $98 \%$ of the cases. The most common symptoms of an infusionrelated reaction included dyspnea, cough, chills, and nausea. The most common severe signs and symptoms included hypertension and dyspnea [see Adverse Reactions (6.1)]

To decrease the risk and severity of infusion-related reactions, premedicate patients prior to SARCLISA infusion with acetaminophen, $\mathrm{H} 2$ antagonists, diphenhydramine, or equivalent; dexamethasone [see Dosage and Administration (2.2)]. Monitor vital signs frequently during the entire SARCLISA infusion. For patients with grade 1 or 2 reactions, interrupt SARCLISA infusion and provide appropriate medica support. If symptoms improve, restart SARCLISA infusion at half of the initial infusion rate, with supportive care as needed and closely monitor patients. If symptoms do not recur after 30 minutes, the infusion rate may be increased to the initia rate, and then increased incrementally, as shown in Table 2 [see Dosage and Administration (2.5)]. In case symptoms do not improve or recur after interruption, permanently discontinue SARCLISA and institute appropriate management. Permanently discontinue SARCLISA therapy if a grade 3 or higher infusion-related reaction occurs and institute appropriate medical management.

\subsection{Neutropenia}

SARCLISA may cause neutropenia. Neutropenia (reported as laboratory abnormality) occurred in $96 \%$ of patients and grade 3-4 neutropenia occurred in $85 \%$ of patients treated with SARCLISA, pomalidomide, and dexamethasone (Isa-Pd). Febrile neutropenia occurred in $12 \%$ of patients and neutropenic infections, defined as infection with concurrent grade $\geq 3$ neutropenia, occurred in $25 \%$ of patients treated with Isa-Pd. The most frequent neutropenic infections included those of upper respiratory tract $(10 \%)$, lower respiratory tract (9\%), and urinary tract (3\%) [see Adverse Reactions (6.1)

Monitor complete blood cell counts periodically during treatment. Consider the use of antibiotics and antiviral prophylaxis during treatment. Monitor patients with neutropenia for signs of infection. In case of grade 4 neutropenia delay SARCLISA dose until neutrophil count recovery to at least $1.0 \times 10^{9} / \mathrm{L}$, and provide supportive care with growth factors, according to institutional guidelines. No dose reductions of SARCLISA are recommended.

\subsection{Second Primary Malignancies}

Second primary malignancies were reported in 3.9\% of patients in the SARCLISA, pomalidomide and dexamethasone (Isa-Pd) arm and in $0.7 \%$ of patients in the pomalidomide and dexamethasone $(\mathrm{Pd})$ arm, and consisted of skin squamous cell carcinoma ( $2.6 \%$ of patients in the Isa-Pd arm and in $0.7 \%$ of patients in the Pd arm), breast angiosarcoma $(0.7 \%$ of patients in the Isa-Pd arm) and myelodysplastic syndrome
( $0.7 \%$ of patients in the Isa-Pd arm). With the exception of the patient with myelodysplastic syndrome, patients were able to continue SARCLISA treatment. Monitor patients for the development of second primary malignancies, as per International Myeloma Working Group (IMWG) guidelines.

\subsection{Laboratory Test Interference}

Test)

SARCLISA binds to CD38 on red blood cells (RBCs) and may result in a false positive indirect antiglobulin tes (indirect Coombs test). In ICARIA-multiple myeloma (MM) the indirect antiglobulin test was positive during SARCLISA treatment in $67.7 \%$ of the tested patients. In patients with a positive indirect antiglobulin test, blood transfusions were administered without evidence of hemolysis. ABO/RhD typing was not affected by SARCLISA treatment. Before the first SARCLISA infusion, conduct blood type and screen tests on SARCLISA-treated patients. Consider phenotyping prior to starting SARCLISA treatment. If treatment with SARCLISA has already started, inform the blood bank that the patient is receiving SARCLISA and SARCLISA interference with blood compatibility testing can be resolved using dithiothreitol-

reated RBCs. If an emergency transfusion is required, noncross-matched ABO/RhD-compatible RBCs can be given as per local blood bank practices [see Drug Interactions (7.1)]. interference with Serum Protein Electrophoresis and

\section{mmunofixation Tests}

SARCLISA is an IgG kappa monoclonal antibody that can be incidentally detected on both serum protein electrophoresis and immunofixation assays used for the clinical monitoring of endogenous M-protein. This interference can impact the accuracy of the determination of complete response in some patients with lgG kappa myeloma protein [see Drug Interactions (7.1)].

\subsection{Embryo-Fetal Toxicity}

Based on the mechanism of action, SARCLISA can cause feta harm when administered to a pregnant woman. SARCLISA may cause fetal immune cell depletion and decreased bone density. Advise pregnant women of the potential risk to a fetus. Advise females with reproductive potential to use an effective method of contraception during treatment with SARCLISA and for at least 5 months after the last dose [see Use in Specific Populations $(8.1,8.3)]$. The combination of SARCLISA with pomalidomide is contraindicated in pregnant women because pomalidomide may cause birth defects and death of the unborn child. Refer to the pomalidomide

prescribing information on use during pregnancy.

\section{ADVERSE REACTIONS}

The following clinically significant adverse reactions from SARCLISA are also described in other sections of the labeling: - Infusion-Related Reactions [see Warnings and Precautions (5.1)]

- Neutropenia [see Warnings and Precautions (5.2)]

- Second Primary Malignancies [see Warnings and Precautions (5.3)]

\subsection{Clinical Trials Experience}

Because clinical trials are conducted under widely varying conditions, adverse reaction rates observed in the clinical trials of a drug cannot be directly compared to rates in the clinical trials of another drug and may not reflect the rates observed in practice.

Multiple Myeloma

The safety of SARCLISA was evaluated in ICARIA-MM a randomized, open-label clinical trial in patients with previously treated multiple myeloma. Patients were eligible for inclusion if they had ECOG status of $0-2$, platelets $\geq 75,000$ cells $/ \mathrm{mm}^{3}$, absolute neutrophil count $\geq 1 \times 10^{9} / \mathrm{L}$, creatinine clearance $\geq 30 \mathrm{~mL} / \mathrm{min}$ (MDRD formula), and AST and/ or ALT $\leq 3 \times$ ULN. Patients received SARCLISA $10 \mathrm{mg} / \mathrm{kg}$ intravenously, weekly in the first cycle and every two weeks thereafter, in combination with pomalidomide and low dose dexamethasone (Isa-Pd) $(n=152)$ or pomalidomide and low dose dexamethasone (Pd) (n=149) [see Clinical Studies (14) in the full prescribing information]. Among patients receiving sa-Pd, 66\% were exposed to SARCLISA for 6 months or longer and $24 \%$ were exposed for greater than 12 months or longer. The median age of patients who received Isa-Pd was 68 years (range 36-83); 58\% male, 76\% white, and 14\% Asian. Serious adverse reactions occurred in $62 \%$ of patients receiving Isa-Pd. Serious adverse reactions in $>5 \%$ of patients who received lsa-Pd included pneumonia (26\%), upper respiratory tract infections (7\%), and febrile neutropenia $(7 \%)$. Fatal adverse reactions occurred in $11 \%$ of patients those that occurred in more than $1 \%$ of patients were pneumonia and other infections [3\%]).

Permanent discontinuation due to an adverse reaction (grades 1-4) occurred in 7\% of patients who received Isa-Pd. The most frequent adverse reactions requiring permanent discontinuation in patients who received Isa-Pd were infections (2.6\%). In addition, SARCLISA alone was discontinued in 3\% of patients due to infusion-related reactions.

Dosage interruptions due to an adverse reaction occurred in $31 \%$ of patients who received SARCLISA. The most frequent adverse reaction requiring dosage interruption was infusionrelated reaction (28\%). 
The most common adverse reactions ( $\geq 20 \%$ ) were neutropenia, infusion-related reactions, pneumonia, upper respiratory tract infection, and diarrhea.

Table 3 summarizes the adverse reactions in ICARIA-MM.

Table 3: Adverse Reactions ( $\mathbf{1 0 \%}$ ) in Patients

Receiving SARCLISA, Pomalidomide, and Dexamethasone with a Difference Between Arms of $\geq 5 \%$ Compared to Control Arm in ICARIA-MIM Trial

\begin{tabular}{|c|c|c|c|c|c|c|}
\hline \multirow[t]{2}{*}{$\begin{array}{l}\text { Adverse } \\
\text { Reactions }\end{array}$} & \multicolumn{3}{|c|}{$\begin{array}{c}\text { SARCLISA+ } \\
\text { Pomalidomide + } \\
\text { Dexamethasone (Isa-Pd) } \\
(\mathrm{N}=152)\end{array}$} & \multicolumn{3}{|c|}{$\begin{array}{c}\text { Pomalidomide + } \\
\text { Dexamethasone (Pd) } \\
\text { (N=149) }\end{array}$} \\
\hline & $\begin{array}{c}\text { All } \\
\text { grades } \\
(\%)\end{array}$ & $\begin{array}{c}\text { Grade } \\
3 \\
(\%)\end{array}$ & $\begin{array}{c}\text { Grade } \\
4 \\
(\%)\end{array}$ & $\begin{array}{c}\text { All } \\
\text { grades } \\
(\%)\end{array}$ & $\begin{array}{c}\text { Grade } \\
3 \\
(\%)\end{array}$ & $\begin{array}{c}\text { Grade } \\
4 \\
(\%)\end{array}$ \\
\hline $\begin{array}{l}\text { Infusion-related } \\
\text { reaction }\end{array}$ & 38 & 1.3 & 1.3 & 0 & 0 & 0 \\
\hline \multicolumn{7}{|l|}{ Infections } \\
\hline Pneumonia* & 31 & 22 & 3.3 & 23 & 16 & 2.7 \\
\hline $\begin{array}{l}\text { Upper } \\
\text { respiratory tract } \\
\text { infection }\end{array}$ & 57 & 9 & 0 & 42 & 3.4 & 0 \\
\hline
\end{tabular}

\begin{tabular}{|l|c|c|c|c|c|c|}
\hline \multicolumn{6}{|l|}{ Blood and lymphatic system disorders } \\
\hline $\begin{array}{l}\text { Febrile } \\
\text { neutropenia }\end{array}$ & 12 & 11 & 1.3 & 2 & 1.3 & 0.7 \\
\hline
\end{tabular}

Respiratory, thoracic and mediastinal disorders

\begin{tabular}{|l|l|l|l|l|l|l|}
\hline Dyspnea $^{\ddagger}$ & 17 & 5.0 & 0 & 12 & 1.3 & 0 \\
\hline
\end{tabular}

\begin{tabular}{|l|c|c|c|c|c|c|}
\hline \multicolumn{7}{|l|}{ Gastrointestinal disorders } \\
\hline Diarrhea & 26 & 2 & - & 19 & 0.7 & - \\
\hline Nausea & 15 & 0 & - & 9 & 0 & - \\
\hline Vomiting & 12 & 1.3 & - & 3.4 & 0 & - \\
\hline
\end{tabular}

CTCAE version 4.03

*Pneumonia includes atypical pneumonia, bronchopulmonary aspergillosis, pneumonia, pneumonia haemophilus, pneumonia influenzal, pneumonia pneumococcal, pneumonia streptococcal, pneumonia viral, candida pneumonia, pneumonia bacterial, haemophilus infection, lung infection, pneumonia fungal, and pneumocystis jirovecii pneumonia.

+Upper respiratory tract infection includes bronchiolitis, bronchitis, bronchitis viral, chronic sinusitis,

fungal pharyngitis, influenza-like illness, laryngitis, nasopharyngitis, parainfluenzae virus infection, pharyngitis, respiratory tract infection, respiratory tract infection viral, rhinitis, sinusitis, tracheitis, upper respiratory tract infection, and upper respiratory tract infection bacterial.

†Dyspnea includes dyspnea, dyspnea exertional, and dyspnea at rest.

Table 4 summarizes the hematology laboratory abnormalities in ICARIA-MM.

Table 4: Treatment Emergent Hematology Laboratory Abnormalities in Patients Receiving Isa-Pd Treatment versus Pd Treatment - ICARIA-MIM

\begin{tabular}{|l|c|c|c|c|c|c|}
\hline $\begin{array}{l}\text { Laboratory } \\
\text { Parameter } \\
\text { n (\%) }\end{array}$ & \multicolumn{3}{|c|}{$\begin{array}{c}\text { SARCLISA+ } \\
\text { Pomalidomide + } \\
\text { Dexamethasone (Isa-Pd) } \\
\text { (N=152) }\end{array}$} & \multicolumn{3}{|c|}{$\begin{array}{c}\text { Pomalidomide + } \\
\text { Dexamethasone (Pd) } \\
\text { (N=149) }\end{array}$} \\
\hline & $\begin{array}{c}\text { All } \\
\text { Grades }\end{array}$ & $\begin{array}{c}\text { Grade } \\
\mathbf{3}\end{array}$ & $\begin{array}{c}\text { Grade } \\
\mathbf{4}\end{array}$ & $\begin{array}{c}\text { All } \\
\text { Grades }\end{array}$ & $\begin{array}{c}\text { Grade } \\
\mathbf{3}\end{array}$ & $\begin{array}{c}\text { Grade } \\
\mathbf{4}\end{array}$ \\
\hline Anemia & 151 & 48 & 0 & 145 & 41 & 0 \\
$(99)$ & $(32)$ & $(28)$ & 0 \\
\hline Neutropenia & 146 & 37 & 92 & 137 & 57 & 46 \\
$(96)$ & $(24)$ & $(61)$ & $(92)$ & $(38)$ & $(31)$ \\
\hline Lymphopenia & 140 & $\begin{array}{c}64 \\
(42)\end{array}$ & $\begin{array}{c}19 \\
(13)\end{array}$ & $\begin{array}{c}137 \\
(92)\end{array}$ & $\begin{array}{c}52 \\
(35)\end{array}$ & $\begin{array}{c}12 \\
(8)\end{array}$ \\
\hline Thrombocytopenia & $\begin{array}{c}127 \\
(84)\end{array}$ & $\begin{array}{c}22 \\
(14)\end{array}$ & $\begin{array}{c}25 \\
(16)\end{array}$ & $\begin{array}{c}118 \\
(79)\end{array}$ & $\begin{array}{c}14 \\
(9)\end{array}$ & $\begin{array}{c}22 \\
(15)\end{array}$ \\
\hline
\end{tabular}

Description of Selected Adverse Reactions Infusion-related reactions

In ICARIA-MM, infusion-related reactions (defined as adverse reactions associated with the SARCLISA infusions, with an onset typically within 24 hours from the start of the infusion) were reported in 58 patients (38\%) treated with SARCLISA. All patients who experienced infusion-related reactions, experienced them during the 1st infusion of SARCLISA, with 3 patients (2\%) also having infusion-related reactions at their 2nd infusion, and 2 patients (1.3\%) at their 4th infusion.

Grade 1 infusion-related reactions were reported in $3.9 \%$, Grade 2 in $32 \%$, Grade 3 in $1.3 \%$, and Grade 4 in 1.3\% of the patients. Signs and symptoms of Grade 3 or higher infusion-related reactions included dyspnea, hypertension, and bronchospasm. The incidence of infusion interruptions because of infusion-related reactions was $29.6 \%$. The median time to infusion interruption was 55 minutes.

In a separate study (TCD 14079 Part B) with SARCLISA 10 $\mathrm{mg} / \mathrm{kg}$ administered from a $250 \mathrm{~mL}$ fixed-volume infusion in combination with Pd, infusion-related reactions (all Grade 2) were reported in $40 \%$ of patients, at the first administration, the day of the infusion. Overall, the infusion-related reactions of SARCLISA $10 \mathrm{mg} / \mathrm{kg}$ administered as a $250 \mathrm{~mL}$ fixed-volume infusion were similar to that of SARCLISA as administered in ICARIA-MM.

Infections

In ICARIA-MM, the incidence of Grade 3 or higher infections was $43 \%$ in Isa-Pd group. Pneumonia was the most commonly reported severe infection with Grade 3 reported in $22 \%$ of patients in Isa-Pd group compared to $16 \%$ in $\mathrm{Pd}$ group, and Grade 4 in $3.3 \%$ of patients in Isa-Pd group compared to $2.7 \%$ in $\mathrm{Pd}$ group. Discontinuations from treatment due to infection were reported in $2.6 \%$ of patients in Isa-Pd group compared to $5.4 \%$ in Pd group. Fatal infections were reported in $3.3 \%$ of patients in Isa-Pd group and in $4 \%$ in Pd group.

\subsection{Immunogenicity}

As with all therapeutic proteins, there is a potential for immunogenicity. The detection of antibody formation is highly dependent on the sensitivity and specificity of the assay. Additionally, the observed incidence of antibody (including neutralizing antibody) positivity in an assay may be influenced by several factors, including assay methodology, sample handling, timing of sample collection, concomitant medications, and underlying disease. For these reasons, comparison of the incidence of antibodies in the studies described below with the incidence of antibodies in other studies or to other isatuximab-irfc products may be misleading.

In ICARIA-MM, no patients tested positive for antidrug antibodies (ADA). Therefore, the neutralizing ADA status was not determined. Overall, across 6 clinical studies in multiple myeloma (MM) with SARCLISA single agent and combination therapies including ICARIA-MM $(\mathrm{N}=564)$, the incidence of treatment emergent ADAs was $2.3 \%$. No clinically significant differences in the pharmacokinetics, safety, or efficacy of

isatuximab-irfc were observed in patients with ADAs.

\section{DRUG INTERACTIONS}

\subsection{Laboratory Test Interference}

\section{Interference with Serological Testing}

SARCLISA, an anti-CD38 antibody, may interfere with blood bank serologic tests with false positive reactions in indirect antiglobulin tests (indirect Coombs tests), antibody detection (screening) tests, antibody identification panels, and antihuman globulin crossmatches in patients treated with SARCLISA [see Warnings and Precautions (5.4)].

Interference with Serum Protein Electrophoresis and Immunofixation Tests

SARCLISA may be incidentally detected by serum protein electrophoresis and immunofixation assays used for the monitoring of $\mathrm{M}$-protein and may interfere with accurate

response classification based on International Myeloma Working Group (IMWG) criteria [see Warnings and Precautions (5.4)].

\section{USE IN SPECIFIC POPULATIONS}

\subsection{Pregnancy}

\section{Risk Summary}

SARCLISA can cause fetal harm when administered to a pregnant woman. The assessment of isatuximab-irfcassociated risks is based on the mechanism of action and data from target antigen CD38 knockout animal models (see Data). There are no available data on SARCLISA use in pregnant women to evaluate for a drug-associated risk of major birth defects, miscarriage or adverse maternal or fetal outcomes. Animal reproduction toxicity studies have not been conducted with isatuximab-irfc. The estimated background risk of major birth defects and miscarriage for the indicated population is unknown. All pregnancies have a background risk of birth defect, miscarriage, or other adverse outcomes. In the U.S. general population, the estimated background risk of major birth defects and miscarriage in clinically recognized pregnancies is $2 \%$ to $4 \%$ and $15 \%$ to $20 \%$, respectively. The combination of SARCLISA and pomalidomide is contraindicated in pregnant women because pomalidomide may cause birth defects and death of the unborn child. Refer to the pomalidomide prescribing information on use during pregnancy. Pomalidomide is only available through a REMS program.

\section{Clinical Considerations}

Fetal/neonatal reactions

Immunoglobulin G1 monoclonal antibodies are known to cross the placenta. Based on its mechanism of action, SARCLISA may cause depletion of fetal CD38-positive immune cells and decreased bone density. Defer administration of live vaccines to neonates and infants exposed to SARCLISA in utero until a hematology evaluation is completed. (isatuximab-irfc) injection, for intravenous use

\section{Data}

Mice that were genetically modified to eliminate all $\mathrm{CD} 38$ expression (CD38 knockout mice) had reduced bone density which recovered 5 months after birth. Data from studies using CD38 knockout animal models also suggest the involvement of CD38 in regulating humoral immune responses (mice), feto-maternal immune tolerance (mice), and early embryonic development (frogs).

\subsection{Lactation}

\section{Risk Summary}

There are no available data on the presence of isatuximab-irfc in human milk, milk production, or the effects on the breastfed child. Maternal immunoglobulin $\mathrm{G}$ is known to be present in human milk. The effects of local gastrointestinal exposure and limited systemic exposure in the breastfed infant to SARCLISA are unknown. Because of the potential for serious adverse reactions in the breastfed child from isatuximab-irfc administered in combination with pomalidomide and dexamethasone, advise lactating women not to breastfeed during treatment with SARCLISA. Refer to pomalidomide prescribing information for additional information.

8.3 Females and Males of Reproductive Potential Pregnancy Testing

With the combination of SARCLISA with pomalidomide, refer to the pomalidomide labeling for pregnancy testing requirements prior to initiating treatment in females of reproductive potential

Contraception

Females

SARCLISA can cause fetal harm when administered to a pregnant woman [see Use in Specific Populations (8.1)] Advise female patients of reproductive potential to use effective contraception during treatment and for at least 5 months after the last dose of SARCLISA. Additionally, refer to the pomalidomide labeling for contraception requirements prior to initiating treatment in females of reproductive potential.

Males

Refer to the pomalidomide prescribing information.

8.4 Pediatric Use

Safety and effectiveness in pediatric patients have not been established.

8.5 Geriatric Use

Of the total number of subjects in clinical studies of

SARCLISA, 53\% (306 patients) were 65 and over, while 14\% 82 patients) were 75 and over. No overall differences in safety or effectiveness were observed between subjects 65 and over and younger subjects, and other reported clinical experience has not identified differences in responses between the adults 65 years and over and younger patients, but greater sensitivity f some older individuals cannot be ruled out.

\section{OVERDOSAGE}

There is no known specific antidote for SARCLISA overdose. In the event of overdose of SARCLISA, monitor the patients or signs or symptoms of adverse effects and take all appropriate measures immediately.

Manufactured by:

sanofi-aventis U.S. LLC

Bridgewater, NJ 08807

A SANOFI COMPANY

U.S. License No. 1752

SARCLISA is a registered trademark of Sanofi

(C)2020 sanofi-aventis U.S. LLC

ISA-BPLR-SA-MAR2O

Revised: March 2020 


\title{
Screening for Phase 1 Clinical Trials: An Opportunity to Evaluate Psychological States and Re- educate Patients on Prognosis?
}

Sarah Moreland, MD, and Shiraj Sen, MD, PhD

\begin{abstract}
Disease progression or recurrence after a period of remission can be a challenging event for individuals seeking cancer treatment. Those referred for possible phase 1 trial enrollment are often motivated to participate in these studies with hope for a cure despite approximately $5 \%$ response rates in this setting. Addressing such commonly held misunderstandings during the initial evaluation for phase 1 trial eligibility could provide a valuable opportunity to improve physician communication by identifying signs of distress or psychiatric conditions, addressing underlying psychological biases, and encouraging adaptive coping strategies.
\end{abstract}

\section{Introduction}

The primary objective of phase 1 clinical trials is to determine the maximally tolerated dose of a drug under investigation. ${ }^{1}$ While historically only $\sim 5 \%$ of subjects enrolled in phase 1 trials demonstrate a radiographic response to the drug of interest, ${ }^{1-4}$ past findings have suggested that individuals are often not always aware of these data. Additionally, while the treatment of metastatic disease is done with palliative intent, to attempt to improve quality or quantity of life, many individuals enrolling in these trials have previously cited cure as a common motivation for participation. ${ }^{1-8}$ Past findings suggest that this discrepancy may be a result of inadequate physician communication ${ }^{9,10}$ and psychological defense mechanisms employed by those facing serious illness. ${ }^{11-13}$ Improving physician communication during the initial stages of phase 1 trial enrollment would not only allow for an increased awareness of the significant psychosocial impact of cancer on an individual but would also provide an opportunity to address prognostic understanding.

\section{The Psychosocial Impact of Cancer}

A diagnosis of cancer poses a unique set of challenges that encompass not only physical manifestations and adverse effects of treatment, but also raises a variety of psychosocial issues. The National
Comprehensive Cancer Network has developed guidelines for the identification and management of distress, defined as "a multifactorial, unpleasant experience of a psychological (ie, cognitive, behavioral, emotional), social, spiritual, and/or physical nature that may interfere with one's ability to cope effectively with cancer, its physical symptoms, and its treatment." 14 An estimated $20 \%$ to $52 \%$ of individuals with cancer struggle with distress, ${ }^{15-17}$ and about one-third meet clinical criteria for depression. ${ }^{18}$

Negative psychological states not only impact overall quality of life in those with cancer, but may also impact overall health. In a study of 151 individuals with metastatic non-small cell lung cancer, $14 \%$ were found to meet criteria for major depression, which was correlated with worse survival. ${ }^{19} \mathrm{~A}$ separate study of 125 women with metastatic breast cancer participating in expressive group therapy showed that those with improving scores on the Center for Epidemiologic Studies-Depression Scale survived 53.6 months whereas women with worsening scores survived 25.1 months. ${ }^{20}$ These findings demonstrate the prognostic value in screening and managing negative psychological states in those with cancer.

In phase 1 trial participants, the presence of hope has been shown to have a positive impact on psychological well-be- 
ing. An exploratory cross-sectional study of 135 patients with incurable cancer who were deliberating participation in a phase 1 trial showed that the presence of hope was associated with higher rates of optimism and improved quality of life. ${ }^{21}$ The results of other studies support these associations: In one, 179 phase 1 trial subjects showed positive correlations between hopefulness and coping, and a negative association between hope and awareness of prognosis. ${ }^{22}$ A study of 24 patients with metastatic renal cell carcinoma and 22 patients with metastatic melanoma demonstrated a negative correlation between treatment-specific optimism and depression. ${ }^{23}$ For physicians, promoting a sense of hope while communicating realistic expectations for prognosis can be challenging and could potentially have significant ramifications if this balance is not met.

\section{Improving Physician Communication}

Incomplete physician communication during the enrollment process has been identified as a potential source of unrealistic expectations with regard to phase 1 trials. ${ }^{9}, 10$ Multiple studies have demonstrated a shared desire for improved communication by both trial participants and physicians. In a study of 328 phase 1 trial participants, $28 \%$ reported that their physician did not fully address the potential impact that a phase 1 trial could have on quality of life. ${ }^{9}$ A separate study of 37 phase 1 trial subjects stated that overall, their expectation for tumor response and more frequent physician communication was not met during the study. ${ }^{24}$ Physicians participating in a survey exploring the barriers to effective communication during phase 1 trial enrollment report time constraints and the trial participant's hesitance to express concerns as commonly encountered challenges. ${ }^{25}$

A recent article by $\mathrm{McF}$ arland et al explored the unique challenges involved in confronting individuals with cancer and provided examples showing how the dynamic roles of the oncologist can be used to help manage these obstacles. For instance, facilitating trust can allow for effective delivery of news that carries uncertainty about the future. Likewise, promoting stability within the oncologistpatient relationship can help individuals cope with loss; and finally, taking time to emphasize individual accomplishments can help define meaning while identifying overall goals. ${ }^{26}$ The importance of open communication is relevant to those considering phase 1 trial enrollment and can help inform an individual's decision as to whether or not to participate. A survey of phase 1 trial subjects showed that individuals who did not initially express their thoughts regarding trial enrollment and were later able to discuss their fears and concerns experienced increased autonomy with respect to making a decision about enrollment. ${ }^{27}$

While oncologists must often discuss difficult information regarding prognosis and unfavorable outcomes, oncology fellowships have not traditionally provided dedicated training for navigating these complex conversations. ${ }^{28}$ Prior work has demonstrated that effective physician communication leads to better identification of patient preferences regarding treatment, allowing for more individualized care. ${ }^{29}$ Several programs have incorporated dedicated communication training into their curriculums. For instance, a program developed by MD Anderson Cancer Center held monthly sessions that used peer-based learning and roleplaying. These sessions provided a framework for how to approach difficult conversations, putting emphasis on using communication skills that incorporate open-ended questions to explore what the patient understands about their condition, create space through listening without interruption, encourage empathy as a response to patient emotion, and promote self-reflection after the encounter. The fellows who completed the program found it to be advantageous, according to anonymous responses to surveys; in addition, the program was successfully integrated into a busy fellowship curriculum. ${ }^{30}$ While difficult conversations are commonly encountered in oncology practice, effective physician communication skills can transform them into opportunities to better understand patient preferences and underscore the importance of communication training in oncology fellowship.

\section{Understanding Defense Mechanisms and Coping Strategies When Facing Severe IIIness}

In the face of serious illness, patients often employ psychological defense mechanisms like therapeutic misconception and unrealistic optimism. Therapeutic misconception is the belief on the part of those participating in clinical trials that the study is intended for clinical benefit rather than research purposes, ${ }^{11}$ while unrealistic optimism describes a subject's belief that they are more likely to experience clinical benefit from a clinical trial compared with other participants, despite evidence to the contrary. ${ }^{13}$ While these biases hypothetically provide some degree of psychological protection, they may unfortunately lead to a misunderstanding regarding the purpose of a phase 1 trial and could prevent an individual from having a realistic understanding of their prognosis. Physicians may benefit from looking out for and recognizing the use of defense mechanisms in those undergoing evaluation for possi- 
ble clinical trial enrollment in the interest of setting realistic expectations.

There has been extensive investigation into the types of coping strategies employed by individuals with cancer. These strategies are often characterized by the extent to which an individual perceives that they have control over their environment. For instance, if a patient perceives themselves to have a higher level of control, this typically leads to problem-based coping, where attempts are made to change external factors that are preventing the patient from attaining their goals. Conversely, patients use emotionally based coping mechanisms when they perceive a loss of control; then, they modify their personal goals to better fit their situation. ${ }^{31}$ In individuals with terminal cancer, meaning-based coping has been shown to have positive clinical outcomes, including lower levels of depression and improved quality of life. ${ }^{32}$ This type of coping involves releasing previously held but now unrealistic objectives in the interest of pursuing goals that are more in line with one's situation. ${ }^{31}$ Encouraging this type of coping strategy in individuals who are considering phase 1 trial enrollment could help to both foster a realistic understanding of their prognosis and benefits of the trial, while also promoting hope and encouragement in embracing goals that are more in line with their clinical situation.

\section{Promising Behavioral Therapies for Individuals With Terminal Cancer}

Cognitive behavioral therapy (CBT), a commonly used psychological intervention that is beneficial for a variety of conditions, more recently has been incorporated into distress management for cancer. ${ }^{14}$ While time and transportation may be limiting factors for office-based CBT in individuals with incurable cancer, a randomized controlled trial from 2019 studied the effect of a web-based CBT application in this population and showed a significant decrease in the rates of depression and anxiety. ${ }^{33}$ These findings support the concept that incorporating CBT into clinical practice is feasible, increasing the accessibility of CBT to individuals with progressive cancer who may not be able to tolerate multiple office visits.

Based in CBT principles is Acceptance and Commitment Therapy (ACT), a novel technique that encourages individuals to embrace their experience with cancer in order to define the values most important to them, in the interest of creating realistic, actionable goals. ${ }^{34}$ Studies investigating the effects of ACT therapy in those with cancer have shown promising results. For instance, a randomized controlled trial comparing ACT with CBT in individuals with ovarian cancer demonstrated improved mood and quality of life despite disease progression in the ACT arm. ${ }^{35}$ In several preliminary studies, ACT has been shown to improve depression and psychological distress and is associated with increased quality of life. ${ }^{36}$ Ongoing clinical trials investigating the effectiveness of ACT include CanACT, in terminally ill individuals with cancer, ${ }^{37}$ and BEACHes, in those transitioning to palliative care. ${ }^{38}$

\section{Conclusions}

The presence of hope in phase 1 trial participants is correlated with optimism and improved quality of life. ${ }^{21}$ Not surprisingly, however, there exists an inverse relationship between hope and awareness of prognosis in this population. ${ }^{22}$ This dichotomy often places physicians in the difficult position of having to communicate a poor prognosis to a patient while attempting to preserve their overall psychological well-being. Effective physician communication can maintain this balance through skills that allow for individual patient preferences and concerns to be heard, while also providing the clinician with the opportunity to identify negative psychological states and poor coping strategies. Recognizing individuals who are struggling with distress or coping could lead to earlier referrals to behavioral therapies, which have been shown to be effective in decreasing depression and negative psychological states. ${ }^{37}$ While only a small portion of individuals enrolled in phase 1 clinical trials derive direct clinical benefit from the drug under investigation, the process of deliberating trial enrollment could provide a valuable opportunity to identify negative psychological states and revisit overall goals of care.

FINANCIAL DISCLOSURE: The authors have no significant financial interest in or other relationship with the manufacturer of any product or provider of any service mentioned in this article.

For full reference list, visit cancernetwork.com/Phase1_screening

\section{Moreland}

is a hospitalist with Colorado Permanente Medical Group, Medical Center of Aurora in Aurora, Colorado.

\section{Sen}

is associate director, Drug Development Unit, Sarah Cannon Research Institute at HealthONE in Denver, Colorado. 


\section{Use of Immunotherapy in Patients With Cancer During the COVID-19 Pandemic}

Andrey Soares, MD ${ }^{1,2,3}$; Fabio A. Schutz, MD ${ }^{1,4}$; Diogo Assed Bastos, MD ${ }^{1,5,6}$; Denis Jardim, MD, PhD ${ }^{1,5}$; Fernando Sabino Marques Monteiro, MD 17,8,9

'Latin American Cooperative Oncology Group, Porto Alegre, Rio Grande do Sul, Brazil.; ${ }^{2}$ Department of Oncology, Hospital Israelita Albert Einstein, Sao Paulo, Sao Paulo, Brazil.; ${ }^{3}$ Department of Oncology, Centro Paulista de Oncologia-Oncoclinicas, Sao Paulo, Sao Paulo, Brazil., "Beneficência Portuguesa de Sao Paulo, Sao Paulo, Sao Paulo, Brazil., "'Department of Oncology, Hospital Sirio-Libanês, Sao Paulo, Sao Paulo, Brazil.; ' ${ }^{\prime}$ nstituto do Câncer do Estado de Sao Paulo, Sao Paulo, Brazil.; 'Department of Oncology and Hematology, Hospital Santa Lucia, Brasilia, Brazil.; ${ }^{8}$ Department of Oncology, Hospital Universitário de Brasilia, Brasilia, Brazil.; ' School of Medicine, Pontifícia Universidade Católica do Rio Grande do Sul, Porto Alegre, Rio Grande do Sul, Brazil.

THE

CASE

In an asymptomatic 77-yearold woman, former 55 packyears smoker, a routine X-ray showed a $45-\mathrm{mm}$ superior left lobe lesion (Figure 1). A chest CT scan confirmed a 36-mm superior left lobe lesion and an aortic-pulmonary lymph node enlargement measuring 42 $\mathrm{mm}$, suspicious for neoplasia (Figure 2). A PET-CT scan showed an elevated uptake in the primary lesion, in the aortic-pulmonary lymph node, and in the left hilar lymph node with a standardized uptake value -40 and 4.3, respectively (Figure 3).

CT-guided lung biopsy showed a lung squamous cell carcinoma. An endobronchial ultrasound-guided transbronchial needle aspiration for lymph-node staging was negative for lymph node spread. Brain MRI was negative. Final staging was determined to be a IIIA (T2bN2) squamous cell carcinoma of the lung.

After multidisciplinary team consultation, the patient received radiotherapy (Figure 4) with concurrent weekly paclitaxel and carboplatin. The treatment was well tolerated, and the patient had no toxicity. A CT scan after therapy showed a good response (Figure 5). After chemo-radiotherapy treatment, the patient started durvalumab as consolidation therapy.

Three months after starting durvalumab, the patient developed a dry cough with no other symptoms. Physical examination was normal and oxygen saturation was $96 \%$. The patient denied contact with coronavirus disease 2019 (COVID-19) positive patients and was practicing all measures of COVID-19 protection. A new PET-CT showed an excellent tumor response, with appearance of some ground-glass opacity in the lungs (Figure 6). A polymerase chain reaction (PCR) naso- and oropharyngeal test to detect severe acute respiratory syndrome (SARS) coronavirus 2 (SARS-CoV-2) was negative, as was the full respiratory viral panel. A subsequent durvalumab dose was put on hold to better define the diagnosis. After 48 hours, the patient repeated the
SARS-CoV-2 test; the results were again negative. Due to the COVID-19 pandemic and her very good clinical status, the patient did not receive corticosteroids at this time and was instead monitored every 48 hours with telemedicine. The cough disappeared after 1 week and a new COVID-19 test was negative. Durvalumab was reinitiated 2 weeks later and the patient is still receiving the medication with no new symptoms, with a very good tolerance. After 3 months a new PET-CT (Figure 7) showed an almost complete resolution of the ground-glass opacity, and the patient maintains an excellent response.

\section{Which of the following is true about immune checkpoint inhibitor-related pneumonitis during the COVID-19 pandemic?}

A. Clinical and radiological findings C. The presence of specific antibodies are not enough to distinguish between could help in the differential diagnosis pneumonitis caused by SARS-CoV-2 or by immunotherapy.

B. No good data exist to demonstrate whether immunotherapy is protective or increases the risk of SARS-CoV-2 infection.

between pneumonitis caused by SARS-CoV-2 or by immunotherapy.

D. After SARS-CoV-2 infection, a window of 14 days with no symptoms is not enough to restart immunotherapy.

E. All of the above are true. 


\section{CORRECT ANSWER: E. All of the above are true.}

Continued from page 370

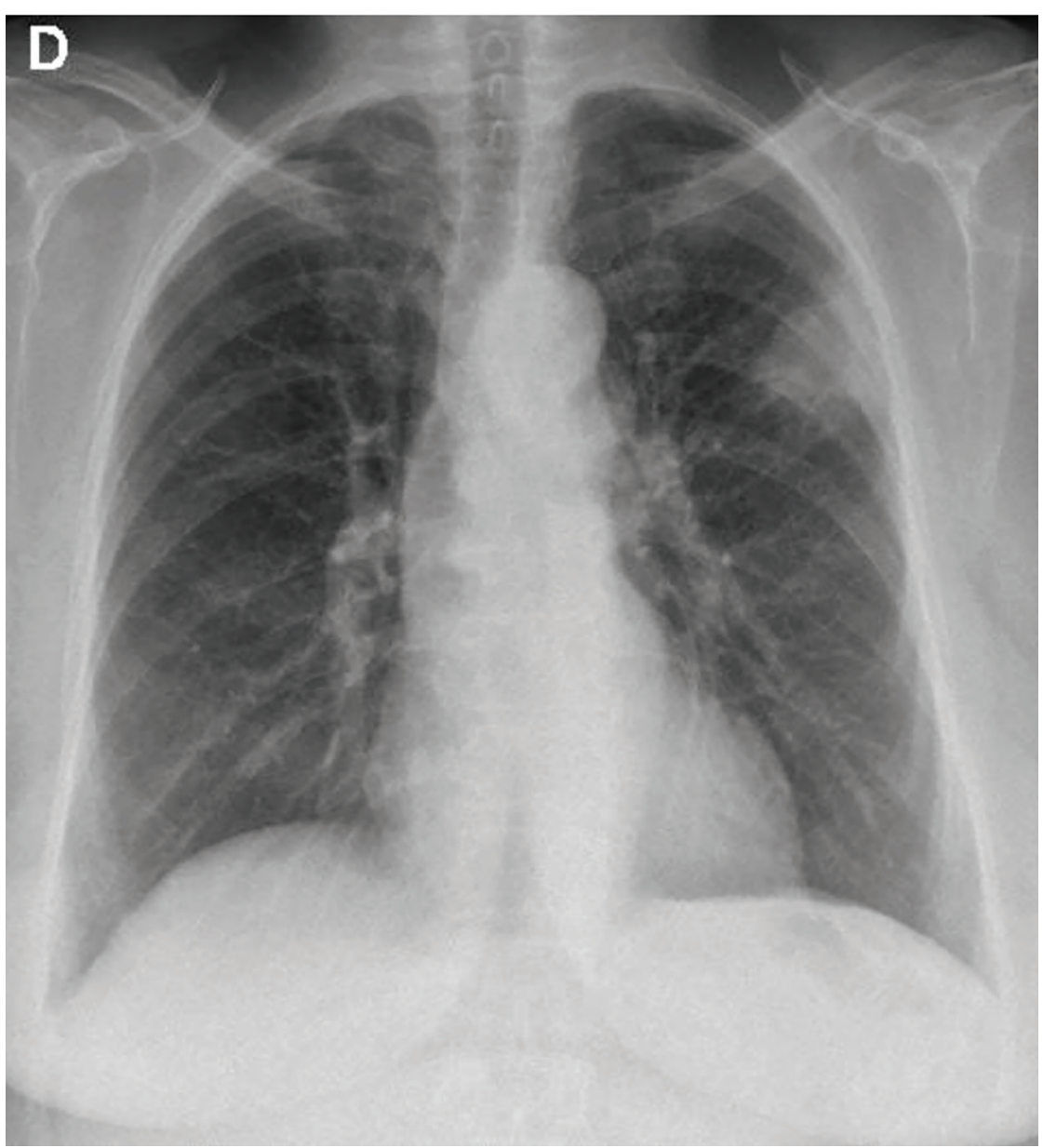

Figure 1. X-ray and the left superior lesion.
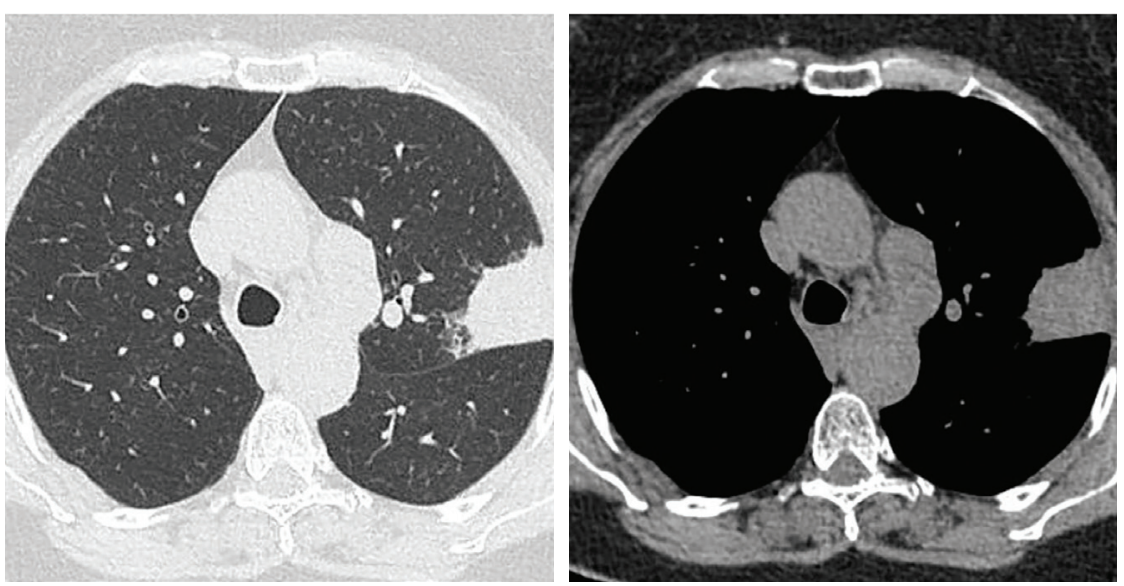

- Figure 2. CT scan with pulmonary lesion and mediastinal lymph node.

\section{Introduction}

SARS-CoV-2 is the virus responsible for the biggest pandemic in recent years, COVID-19. SARS-Cov-2 is a positive sense single-strand RNA virus in the coronaviridae family with a potential to infect humans, including transmission between them. After the first case was reported in Wuhan, China, at the end of 2019, COVID-19 reached global proportions, leading the World Health Organization to declare it a pandemic on March 11, 2020. Globally, COVID-19 infection rates are still rising and as of August 31, $2020,25,402,968$ people were infected in 188 countries, with 849,303 deaths. ${ }^{1,2}$

SARS-CoV-2 causes an asymptomatic or mild infection in approximately $80 \%$ of cases. However, it presents in the other $20 \%$ as a symptomatic and potentially serious infection. The most severe problems caused by SARS-CoV-2 are respiratory failure, due to pneumonitis, and/or multiple organ failure. The evolution of this serious disease is attributed to an exacerbated inflammatory response. ${ }^{2}$ After contamination and contact with the host, SARS-CoV-2 uses angiotensin-converting-enzyme 2 (ACE2) as receptor to cell invasion. During this process, type II transmembrane serine protease (TMPRSS2) expression seems to be important as a coadjuvant protein to assist SARS-CoV-2 in cell invasion. ${ }^{3,4}$ Patients who progress to the severe form of the disease have an inflammatory response that leads to a reduction of the expression of cytotoxic CD8+ and CD4+ T lymphocytes. Also, important inflammatory cytokines such as interleukin-6 (IL-6), tumor necrosis factor- $\alpha$, and interferon- $\gamma$ (IFN- $\mathrm{\gamma}$ ), as well as others, increase. ${ }^{2,4,5}$ In this context, as with other viral infections, increased expression of regulatory molecules of inflammatory response, like PD-1 and PD-L1, occurs. ${ }^{2,4-6}$

\section{COVID19 and Cancer}

The morbidity and mortality of COVID-19 is higher in elderly and male 


\section{TABLE. Main Characteristics of Pneumonitis Caused by Checkpoint Inhibitors and by SARS-CoV-2 Infection}

\begin{tabular}{|c|c|c|}
\hline Pneumonitis & Checkpoint inhibitor & SARS-CoV-2 \\
\hline \multirow[t]{2}{*}{ Epidemiology } & Absence of contact with infected person & Contact with infected person \\
\hline & $\begin{array}{l}\text { Most commonly begins 8-12 weeks after } \\
\text { the start of treatment }\end{array}$ & $\begin{array}{l}\text { Residence in or recent visit to a region with a high number of } \\
\text { COVID-19 cases }\end{array}$ \\
\hline \multirow[t]{2}{*}{ Clinical findings } & Cough, dyspnea, wheezing & \multirow[t]{2}{*}{ Fever, cough, chest tightness, dyspnea, hypo or anosmia } \\
\hline & $\begin{array}{l}\text { Associated or not associated with other } \\
\text { autoimmune manifestations }\end{array}$ & \\
\hline $\begin{array}{l}\text { Early onset of } \\
\text { symptoms }^{23,24}\end{array}$ & $\begin{array}{l}9 \text { days to } 19 \text { months since start of treat- } \\
\text { ment (median, } 2.8 \text { months) }\end{array}$ & 7 to 10 days since start of clinical symptoms \\
\hline \multirow[t]{2}{*}{$\begin{array}{l}\text { Radiological } \\
\text { findings } \mathbf{s}^{25,26}\end{array}$} & $\begin{array}{l}\text { Ground-glass opacities, reticular opaci- } \\
\text { ties, usually multifocal with a peripheral } \\
\text { distribution, mainly in lower or middle } \\
\text { lung }\end{array}$ & \multirow{2}{*}{$\begin{array}{l}\text { * Bilateral multilobar ground-glass opacification with a } \\
\text { peripheral or posterior distribution, mainly in the lower lobes } \\
{ }^{*} \text { Atypical presentation: consolidative opacities superimposed } \\
\text { on ground-glass opacities (particularly in the elderly } \\
\text { population) } \\
{ }^{*} \text { Less common findings: septal thickening, bronchiectasis, } \\
\text { pleural thickening, and subpleural involvement (mainly in the } \\
\text { later stages of the disease) } \\
{ }^{*} \text { Pleural effusion, pericardial effusion, lymphadenopathy, } \\
\text { cavitation, CT halo sign, and pneumothorax are uncommon but } \\
\text { may be seen with disease progression. }\end{array}$} \\
\hline & $\begin{array}{l}\text { Distinct radiographic patterns have been } \\
\text { described: } \\
\text { (a) organizing pneumonia } \\
\text { (b) nonspecific interstitial pneumonia } \\
\text { (c) hypersensitivity pneumonitis } \\
\text { (d) acute interstitial pneumonia-acute } \\
\text { respiratory distress syndrome } \\
\text { (e) bronchiolitis }\end{array}$ & \\
\hline 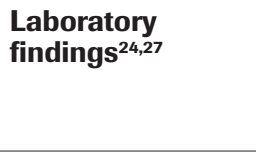 & Unspecified & $\begin{array}{l}\text { Elevated serum D dimer and fibrinogen } \\
\text { Normal aTTP } \\
\text { Elevated serum IL-6 } \\
\text { Lymphopenia }\end{array}$ \\
\hline Diagnostic tests & Not available & $\begin{array}{l}\text { RT-PCR SARS-CoV-2 naso- and/or oropharynx swab } \\
\text { Positive serum antibody lgM }\end{array}$ \\
\hline Clinical evolution & $\begin{array}{l}\text { Quick improvement with corticosteroid } \\
\text { therapy }\end{array}$ & $\begin{array}{l}\text { Prolonged clinical symptoms with frequent need for oxygen } \\
\text { therapy }\end{array}$ \\
\hline
\end{tabular}

COVID-19, coronavirus disease 2019; IgM, immunoglobulin M; IL-6, interleukin-6; RT-PCR, reverse-transcriptase polymerase chain reaction; SARSCoV-2, severe acute respiratory syndrome coronavirus 2; aTTP, activated partial thromboplastin time.

patients, as well as in those with comorbidities. The most relevant comorbidities associated with serious outcomes are hypertension, diabetes, cardiovascular disease, obesity, dementia, chronic respiratory disease, kidney disease, liver disease, autoimmune disease, immunosuppressive disease, and cancer.

Specifically in cancer patients, those who are elderly and/or who have lung cancer, hematologic malignancy, advanced-stage disease, active solid tumor, or a diagnosis of solid tumor in the past 5 years, or who had oncological surgery performed in the last 30 days, are at highest risk of an unfavorable outcome.
With regard to systemic oncological treatments, correlation of the use of immunotherapy with checkpoint inhibitors (anti-PD-1, anti-PD-L1, and antiCTLA-4) with the risk of more severe COVID-19 infection is uncertain. So far, published data are conflicting; some data show serious outcomes in patients receiving immunotherapy, while others do not. . $^{6-12}$

Considering the magnitude of the pandemic, several expert-opinion guidelines have emerged, aiming to minimize both the risk of infection and complications related to COVID-19. Many focused on recommended measures, such as so- cial distancing, and assess the risks and benefits of delaying systemic oncological treatments. Additionally, in clinical practice, many patients have chosen to postpone their oncological treatments. However, evidence shows that cancer-related mortality increases due to delays in cancer diagnosis and treatment, even if such delays might seem warranted due to the COVID-19 threat. $^{13,14}$

\section{COVID-19 Risk in Cancer Patients Receiving Immunotherapy}

In patients with COVID-19, pneumonitis is the main cause of hospitalization 

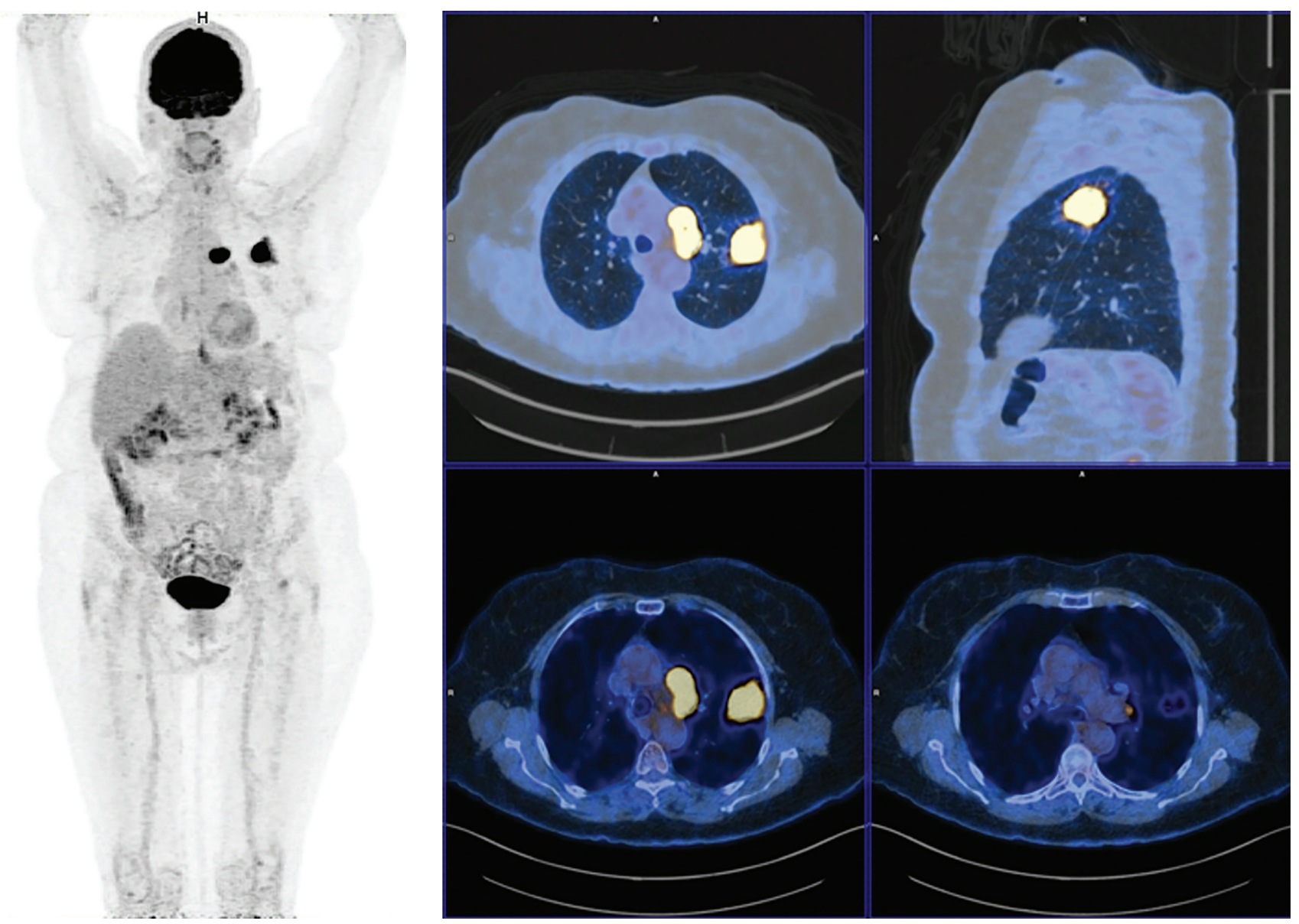

- Figure 3. PET-CT with pulmonary and lymph node involvement.

and death. However, pneumonitis is also an adverse event (AE) caused by immunotherapy (IMT); it occurs in between $2 \%$ and $5 \%$ of patients receiving IMT monotherapy and in about $10 \%$ of patients receiving IMT combination therapy (anti-PD-1/PD-L1 + anti-CTLA-4). Notably, although pneumonitis is not a common AE in patients receiving IMT, it is associated with a high mortality rate (about 35\%). 6,11,15

Clinically, the pneumonitis caused by SARS-CoV-2 and that induced by IMT can be confused, because symptoms such as fever, dry cough, dyspnea, and fatigue are common in both presentations. The reverse-transcriptase PCR (RT-PCR) test to diagnose COVID-19 can register a false negative during the first days of symptoms ${ }^{7,11}$ (Table), and in many localities, results of the test can take more than a few days to be processed. These factors can increase the confusion surrounding the diagnosis and can delay the decision of whether or not to begin treatment for IMT-induced pneumonitis.

Another challenge is that imaging findings from both causes of pneumonitis can be indistinguishable. The most common presentation in COVID-19 patients is the pattern of ground-glass opacities seen on a CT scan of the chest, which is a possible characteristic of IMT-related pneumonitis. ${ }^{6,11,16}$ Therefore, statement $A$ is correct.

During this pandemic, treating patients with cancer using IMT can be challenging, and oncologists face several quandaries. The first is whether IMT could potentially have a protective effect, reducing the risk of SARS-CoV-2 infection because the therapy may enhance patient immunity. However, only observational studies have raised the possibility of the protective effect of IMT, and further data are required to confirm this hypothesis and use it to guide the management of patients with cancer.

Conversely, it is possible that patients receiving IMT may be more susceptible to SARS-CoV-2 infection because of higher expression of ACE2 and TMPRSS2 in the case of naturally or IMT-stimulated inflamed tumors. ${ }^{3-5,12}$ However, previous data do not demonstrate that the use of IMT increases the risk of other viral pneumonias, such as influenza. ${ }^{17}$ Therefore, statement $\mathrm{B}$ is also correct. Yet it is known that in 


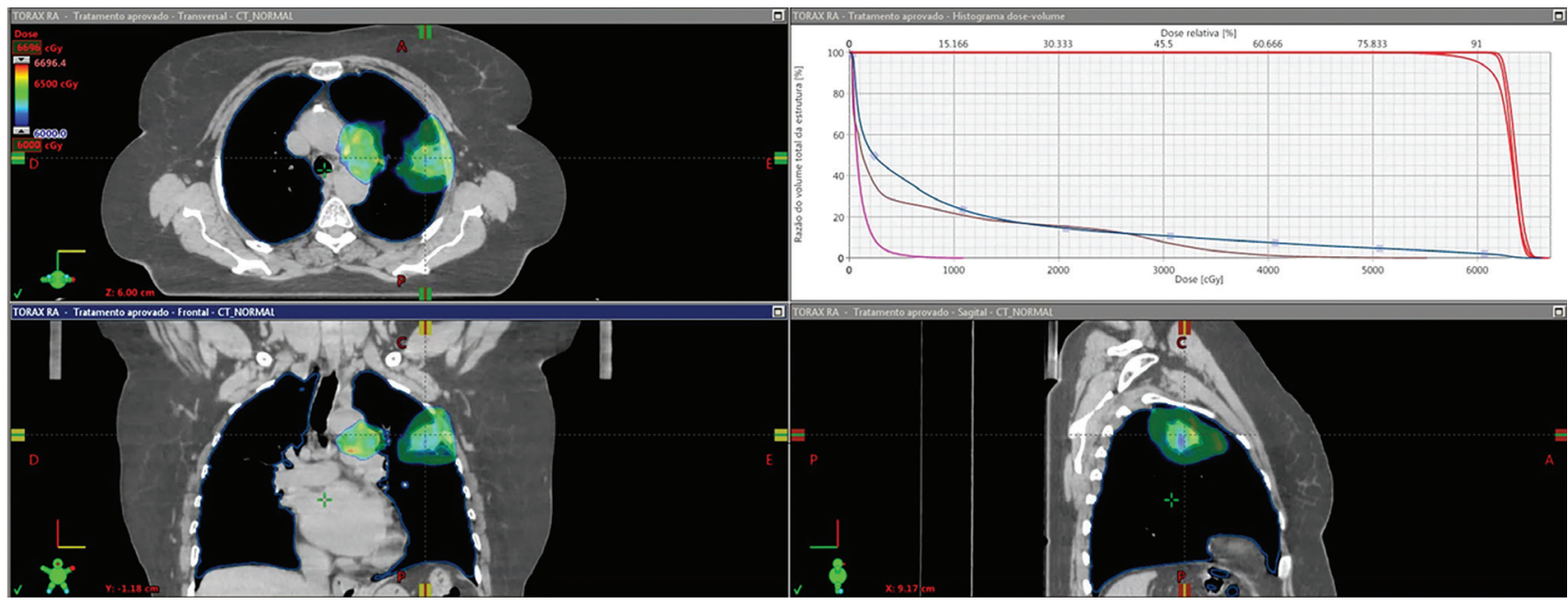

- Figure 4. Radiotherapy plan.

severe cases of pneumonitis, the host experiences exacerbated inflammation, and in these cases, the use of IMT could enhance the inflammatory response. Rarely, the IMT itself can cause cytokine release syndrome, characterized by the release of cytokines including IL- 6 and IFN-y. In the case of SARS-CoV-2 infection in patients receiving IMT, the risk of cytokine release syndrome is high. Thus, the second quandary oncologists face is the challenge to correctly diagnosis SARS-CoV-2 infection or IMT-induced pneumonitis. ${ }^{4,6,11}$

Another important consideration is controlling immune-related AEs in patients who need long-term immunosuppression therapy. These patients must be rigorously followed because of their higher risk of infection. ${ }^{18}$ To help distinguish between COVID-19 and IMT-related pneumonitis when clinical and radiological findings are not sufficient, epidemiological data may be useful. In such cases, only a COVID-19 diagnostic test - the gold standard being the RTPCR nasopharynx and/or oropharynx swab for SARS-CoV-2 ${ }^{19}$ - will provide a definitive diagnosis of COVID-19. Considering that the humoral immune response with immunoglobulin $\mathrm{G}$ ( $\operatorname{IgG})$ antibody production happens, on average, 14 days after symptom onset, and that these antibodies can be detected up
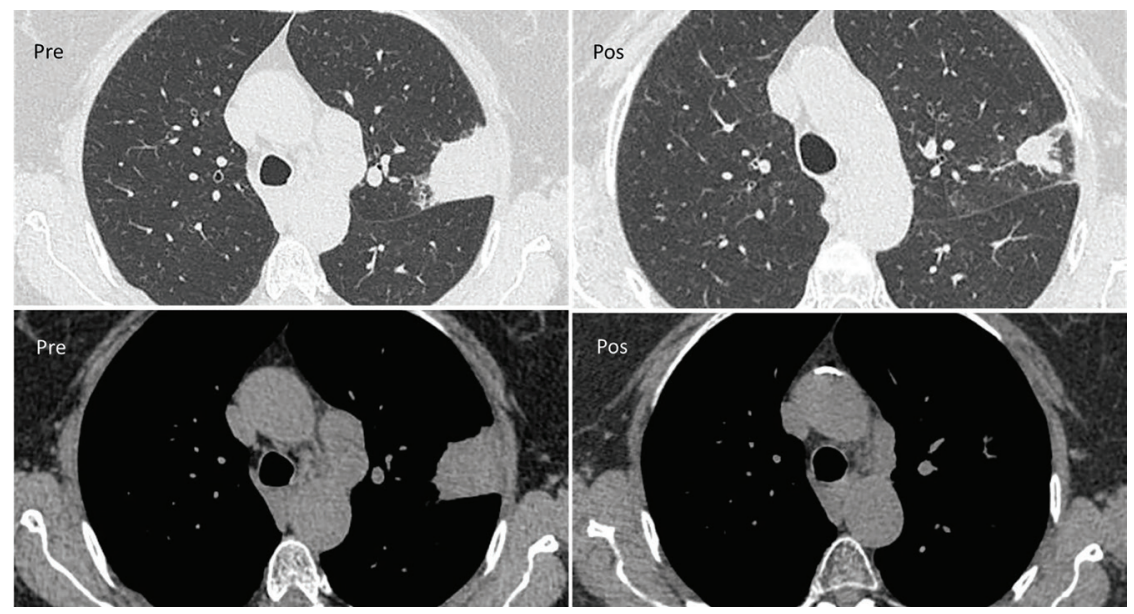

- Figure 5. CT scan prior to radiochemotherapy and post radiochemotherapy.

to 36 months after the infection, another reasonable option to consider when investigating the cause of pneumonitis is the quantitative analysis of serum IgG antibodies in the blood sample. In a clinical setting when a patient presents with respiratory symptoms, the presence of IgG antibodies could be evidence that the patient has already had contact with SARS-CoV-2 and that pneumonitis is probably secondary to IMT. ${ }^{20,21}$ Therefore, statement $\mathrm{C}$ is a correct answer.

In such a case, immediately postpone IMT and begin clinical support. IMT can be restarted as soon as the pneumonitis resolves. The early diagnosis of lung injury is fundamental to define the most appropriate treatment. For moderate IMT-induced pneumonitis, corticosteroid treatment must be started immediately, but for pneumonitis secondary to SARS-CoV-2 infection, corticosteroid treatment has shown benefit only in patients receiving concurrent oxygen therapy; it may actually be harmful in mild cases. ${ }^{11,22}$ In cases of severe pneumonitis, when the patient requires hospital admission and oxygen therapy, it is reasonable to consider starting corticosteroid treatment early; corticosteroids are indicated in severe presentation of pneumonitis regardless of the cause and, in cases of IMT-related pneumonitis, corticosteroids improve symptoms very quickly. 
For patients with SARS-CoV-2-related pneumonitis who are receiving IMT, the recommendation is to restart IMT treatment at least 14 days after symptom onset and only after the patient has been asymptomatic for at least 72 hours. In addition, the patient ideally should have 2 negative RT-PCR test results, 24 hours apart. However, in some localities RT-PCR kits are scarce, and in this scenario, a single negative RT-PCR test result together with clinical improvement may be enough. When it is impossible to repeat the exam, all cases must be discussed with patients, caregivers, and the medical oncologist; local recommendations should also be followed as closely as possible. ${ }^{11,19}$ Therefore, statement D is also a correct answer.

The medical oncology community had previously developed measures to minimize IMT-related pneumonitis. The American Society of Clinical Oncology $^{19}$ has made new recommendations to mitigate the risk to patients with SARSCoV-2 infection who receive oncological treatments; these include performing an RT-PCR test on all patients 48 to 72 hours before the first cycle of systemic therapy; clinical and epidemiological screening for all patients via questionnaire, 48 to 72 hours before each cycle of systemic therapy; and clinical and epidemiological evaluation when the patients arrive at the clinic and/or hospital. In symptomatic patients who are screened before arrival to the oncology unit, the current recommendation is that the patient be tested for SARS-CoV-2 outside the oncology unit. If a patient arrives at the oncology unit with COVID-19 symptoms, they must be isolated, evaluated, and tested for SARS-CoV-2. Facial masking and social distancing should be required of all patients ${ }^{11,19}$ (Figure 8).

\section{Conclusions}

The COVID-19 pandemic may take a long time to be controlled. Until it is, it is vital that we do not fail to recommend and maintain the appropriate treatment for
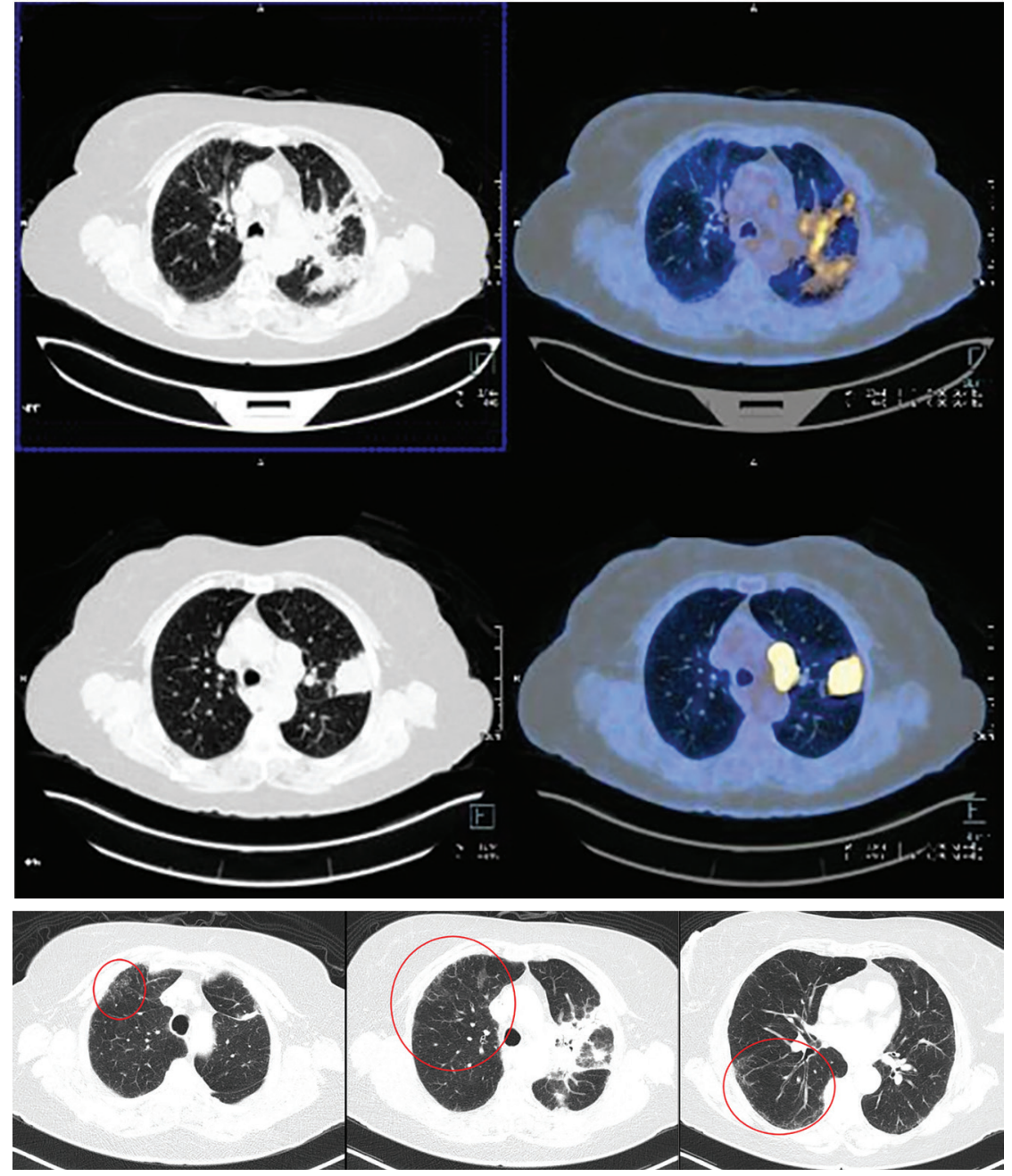

- Figure 6. PET-CT with an excellent tumor response and appearance of ground-glass opacity.
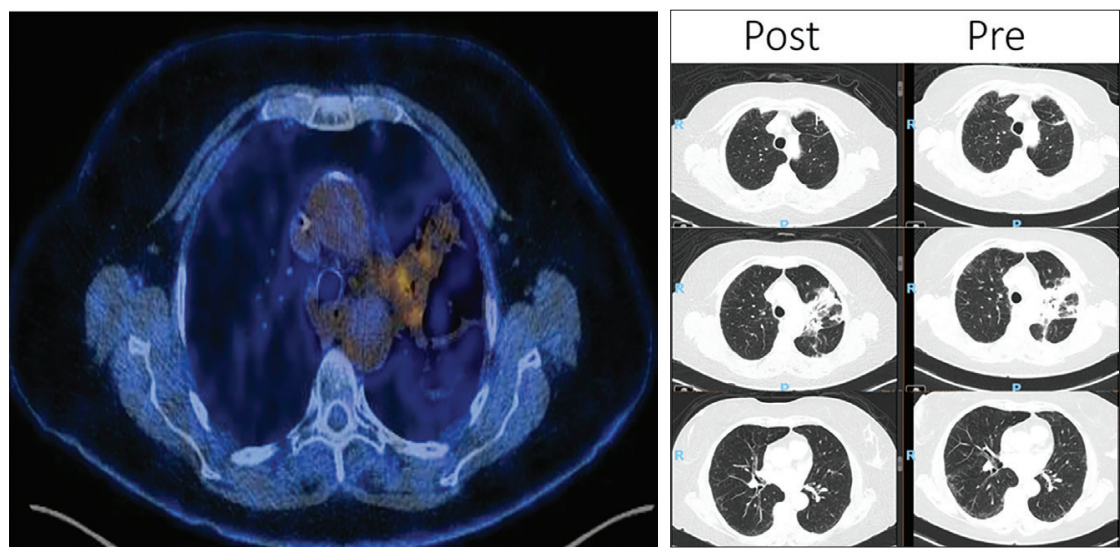

- Figure 7. PET-CT with a tumor good response and resolution of almost all ground-glass opacity. 
patients with cancer. Educating patients, caregivers, and the health care team, and adapting oncology clinics and hospital oncology units to this new situation, is essential. Early reporting of symptoms by patients or caregivers and actively screening for symptoms by the health care team are fundamental to diagnosis and to taking the most appropriate treatment approaches.

\section{CONFLICTS OF INTERESTS: \\ Andrey Soares}

Honoraria: Janssen, Pfizer, Bayer, Novartis, AstraZeneca, Astellas Pharma, Pierre Fabre, Merck Serono, Sanofi, Roche, MSD Consulting or Advisory Role: Astellas Pharma, Janssen, Roche, Bayer, Lilly, AstraZeneca, Novartis, MSD, Bristol Myers Squibb Research Funding: Bristol Myers Squibb (Inst) Travel, Accommodations, Expenses: AstraZeneca, Pfizer, AstellasPharma, Bristol Myers Squibb, Bayer, Roche, Janssen, Merck Serono, Sanofi, Ipsen

\section{Fabio A. Schutz}

Honoraria: Janssen, Bayer, AstraZeneca, Astellas, Roche, MSD, Bristol-Myers Squibb, Ipsen

Consulting or Advisory Role: Astellas, Janssen, Roche, Bayer, AstraZeneca, MSD, Bristol Myers Squibb, Ipsen

Travel, Accommodations, Expenses: Bristol Myers Squibb, MSD, Janssen, Astellas Pharma

\section{Diogo Basto}

Honoraria: Janssen, Pfizer, Bayer, AstraZeneca, Astellas, Merck Serono, Roche, MSD, BMS Consulting or Advisory Role: Astellas, Janssen, Roche, Bayer, AstraZeneca, MSD, Bristol-Myers Squibb Research Funding: Janssen, Astellas, Bayer.

Travel, Accommodations, Expenses:

AstraZeneca, MSD, Bayer, Janssen

\section{Denis Jardim}

Honoraria: Janssen, Pfizer, Bayer, Novartis, AstraZeneca, Astellas Pharma, Merck Serono, Roche, MSD, Bristol Myers Squibb, Libbs Consulting or Advi.sory Role: Janssen, Roche,

- Figure 8. Suggested Management of Patients With Cancer on Immunotherapy Who Develop Respiratory Symptoms. ${ }^{19,28}$

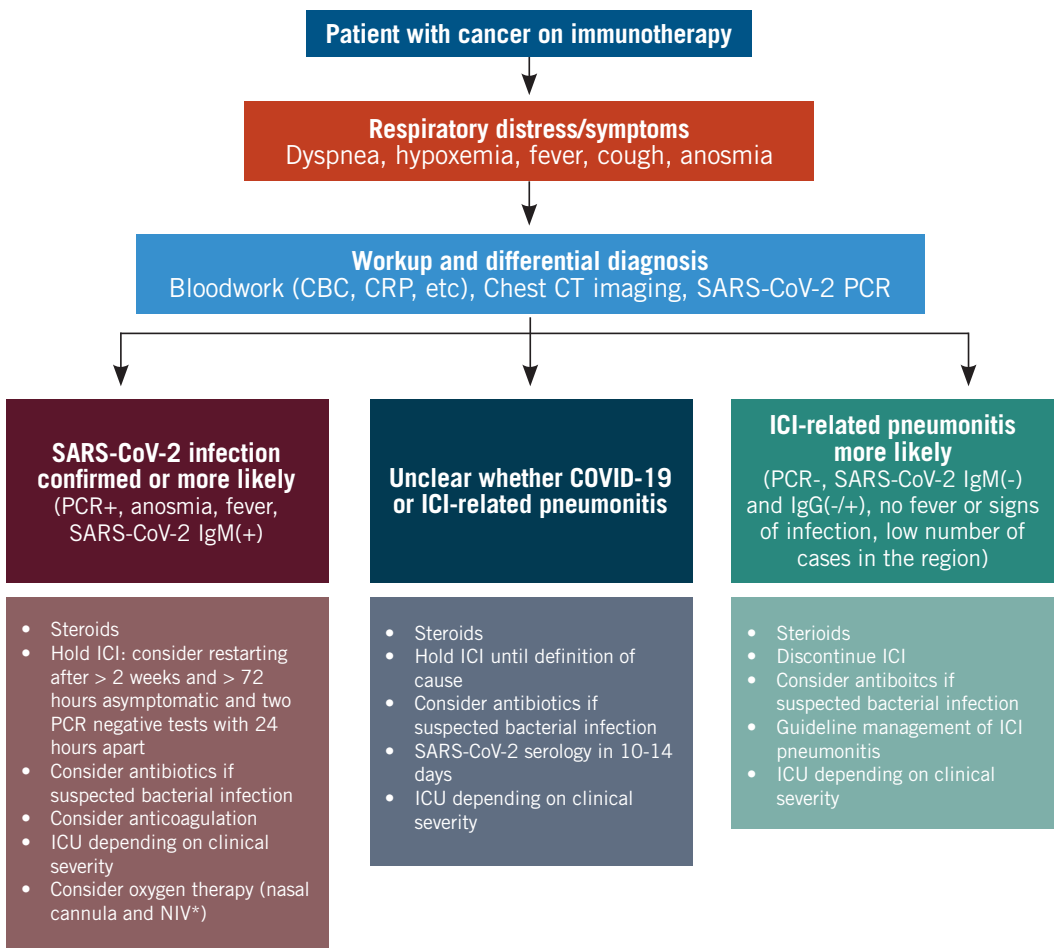

CBC, complete blood count; CRP, C-reactive protein; COVID-19, coronavirus disease 2019; ICl, immune checkpoint inhibitor; IgG, immunoglobulin G; IgM, immunoglobulin M; PCR, polymerase chain reaction; SARS-CoV-2, severe acute respiratory syndrome coronavirus 2 .

AstraZeneca, MSD, Bristol Myers Squibb Research Funding: Janssen, Bristol Myers Squibb, Roche (Inst)

Travel, Accommodations, Expenses: Bristol Myers Squibb, MSD, Janssen

\section{Fernando Sabino M Monteiro}

Honoraria: Janssen, Bayer, Bristol-Myers Squibb, Astellas Pharma, ACHE, Roche, MSD

Consulting or Advi.sory Role: Jan.ssen, Roche, MSD, Bristol-Myers Squibb

Research Funding: Janssen

Travel, Accommodations, Expenses: Bristol Myers Squibb, Roche, Janssen, MSD

\section{ABOUT THE SERIES EDITORS:}

MARIA T. Bourlon, MD is Associate Professor, Head Urologic Oncology Clinic, National Researcher. Instituto Nacional de Ciencias Médicas y Nutrición Salvador Zubirán. Mexico City, Mexico.

E. David Crawford, MD, is Chairman, Prostate Conditions Education Council; Editor in Chief, Grand Rounds in Urology; and Professor of Urology, University of California San Diego, La Jolla, California.

$\rightarrow \quad$ For full reference list, visit cancernetwork.com/IMT_COVID-19
Soares is a medical oncologist at Centro Paulista de Oncologia and Hospital Israelita Albert Einstein.

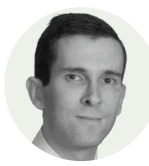

Schutz is a medical oncologist at Beneficência Portuguesa de São Paulo.

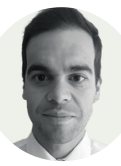

Monteiro is a medical oncologist at Hospital Santa Lucia and Hospital de Universitario de Brasilia.
Jardim is a medical oncologist at Hospital SírioLibanês in São Paulo, Brazil. He is currently the head of clinical research at Sírio-Libanês and coordinator of oncology at the Hospital Unit in Itaim.
Bastos is a genitourinary oncologist at Hospital Sírio-Libanês and Instituto do Cancer do Estado de São Paulo (Sao Paulo Cancer Institute), Brazil. He is currently the chair of the genitourinary branch of the Latin American Cooperative Oncology Group. 


\section{Is Telehealth Here to Stay?}

$\mathrm{T}$ he telehealth explosion was facilitated by the onset of the coronavirus disease 2019 (COVID-19) pandemic, but what happens when the crisis is over? Will there be lasting changes to the practice of medicine and delivery of care, or will providers and patients alike be eager to go back to the "old way" of doing things?

Jeremy Gabrysch, MD, a physician and CEO of Remedy, an on-demand urgent care service that delivers doctors right to your front door, recently spoke with Medical Economics ${ }^{\circledR}$, a sister publication of ONCOLOGY ${ }^{\circledR}$, to discuss what the future of telehealth may hold.

Q:

Do you think

this level of

telehealth use will be

maintained after the

COVID-19 pandemic

ends, or do you expect

it to decline?

GABRYSCH: That's a great question. It is certainly at an incredible level right now in terms of its adoption. When you look at last year, for example, McKinsey \& Company did a study in which they asked people about their interest level regarding telehealth, and engaging their doctor by telehealth; only $11 \%$ said that they were interested in that venue versus in-person. Now, looking at that same question today- "Are you interested in using telehealth to connect with your doctor?"-and $76 \%$ of people say that they're interested. That's a huge jump, definitely affected by what we're seeing with the pandemic.

You also look at the numbers of cases. For example, we're a very virtual-focused company and do a lot of telehealth [under normal conditions]. And even with that in mind, our numbers were up: We're seeing $1000 \%$ increase in the number of telehealth video visits that we're doing relative to this time last year. The spike right now is huge.

So, wondering what's going to happen when this crisis subsides or passes, it's definitely going to decline some from where it is right now. [I think] there will be a tendency to go back to the "old way" of doing things. Providers may be more eager to see patients back in the clinic; patients might even gravitate toward that a little bit. But I don't think that we'll go back [fully] to where we were. And I would say that we really should resist going back at all, because this [pandemic] has presented a huge opportunity. We have moved virtual care forward. We know that we can provide a more connected experience, better outcomes, and just a more virtual-first approach to medicine.

Think about the way you bank, for example. You probably engage with your bank, mostly through your smartphone or your computer, and occasionally you go into the branch. We're not saying that all in-person care will go to virtual, but if you consider the banking analogy, a lot of what you can do can be done remotely, and then occasionally you need those visits to the brick-andmortar branch. I'd submit is that this is an opportunity for us to move medicine more into that paradigm, in which we do a lot of the care virtually, and occasionally bring patients in for in-person visits, when that makes sense.

\section{Q: In the past, telehealth wasn't widely embraced because many payers wouldn't reimburse it at the same level as an office visit, if at all. Do you think the success of telehealth during COVID-19 has permanently changed the reimbursement outlook for the better?}

GABRYSCH: I certainly hope so. I get this question a lot. Before COVID-19 hit, there was a question about whether or not payers were going to pay for virtual care, and I think that question has largely been answered: Payers are saying that they'll pay for it. So, it's not so much a question of whether they will pay for it, but next comes the question of parity. Will payments [for virtual care] be equal to payments for in-office care? I think that's an open question. For these gains in virtual care to be sustained, and for us to really capitalize on this, we've got to solve this issue.

Value-based contracting is one way in which to solve it. If you pay for the total package of care that's being delivered, then the providers are definitely incentivized to provide more care virtually, to provide these sort of virtual touchpoints 
with patients that will probably improve outcomes. Within a value-based construct, they actually have a way to get reimbursed for it. In a fee-for-service structure, we definitely have to think about how we can incentivize this behavior, and how we can reimburse providers so that they actually are incentivized to drive this type of care as we move forward, and not just go backward and lose all the momentum that [has been developed].

\section{Q: Smaller rural practices often struggle with technology because of cost and complexity. What can be done to make sure they aren't left behind with telehealth and some of these other advances?}

GABRYSCH: One big issue here in rural areas is internet connectivity. Fortunately, states and larger organizations are working to improve broadband access and cell phone coverage in many of these areas, because that really is imperative if you're going to be able to deliver care in this way. But I will tell you that in the states we serve with Remedy, we see lots of patients who live in the rural areas, patients who might be a decent drive from a brick-and-mortar facility, but they're able to get connected to care using virtual care. As we look at rural practices, where their patients may be fairly spread out, it may be more difficult for [patients to physically] get in, because of transportation issues, [for example]. So this is a huge opportunity to really transform care for those areas as well. And again, some of it comes back to the reimbursement issue. There are smaller practices in these rural areas who are relying on fee-for-service income, a lot of times from these office visits. We can improve connectivity, we can talk about more connected care, etc, but at the end of the day, we have to figure out a way to reimburse these providers for that type of care in order to incentivize it and make sure that it does, indeed, take off.

\section{"I would say that we should really rethink what the standard of care looks like in light of our access to technology with these new connected devices and virtual meetings."}

\section{Q:}

How will the standards of care change in medicine post COVID-19? What do you think will look different? What will change once this crisis has passed?

GABRYSCH: I think there needs to be an openness in the medical community to what a new standard of care could look like, one that is heavily focused on virtual and leverages virtual [encounters]. I'll give you a couple of examples. First, the other day, I had a friend who was experiencing some heart palpitations. Through her Apple Watch, she was able to obtain a digital electrocardiogram, which was then submitted to cardiologists, virtually. They took a look at it, chatted with her, and then ordered some additional tests to be done. All of that typically would have taken place in an emergency department or urgent care clinic or the cardiologist's office. But because of technology, because of this wearable device and access to virtual care, we're able to envision a new standard of care about what that looks like.

A second example, another specialty care example, involves a patient who had a foot injury. Using high-quality photos and video of their foot, they consulted with an orthopedic surgeon using virtual care. An X-ray was ordered, a small nondisplaced fracture was found in the foot, and the specialist recommended a walking boot for several weeks. Again, all of that care took place virtually. Typically, we would say, "Well, the standard of care should require an in-person visit," but I would challenge that. I would say that we should really rethink what the standard of care looks like in light of our access to technology with these new connected devices and with virtual meetings. Patients have a lot of monitors, on their body and in their homes, that can provide us with a lot of information. We can actually deliver a very high quality of medicine virtually, whereas we might not have been able to do that before.

[Yes,] our medical system is heavily invested in the brick-and-mortar model that got us to where we are today, [but] we have to be careful not to be too saddled by that. If we do, we will be very reluctant to embrace these changes, and we'll be more inclined to go back to the way we used to do things. We're too narrowly focused on that brick-and-mortar model and that old way of doing things, and virtual care can solve so many [problems] for which people typically visit the office. We have an opportunity to really change the way we deliver care, and the way that that we pay for care-actually, [to change] the cost of care. We waste a ton of money in this country, providing in-person office visits and care that don't actually make us healthier, and don't make us live longer. [It represents] a huge chunk of the $\$ 3.5$ trillion that we spend [on health care]. That's just waste. And if we really are open to adopting this more virtual approach, we will save so much money, we will provide a better experience, and we will get better outcomes for patients.

FINANCIAL DISCLOSURE: The authors have no significant financial interest in or other relationship with the manufacturer of any product or provider of any service mentioned in this article.

This article originally appeared online at medicaleconomics.com 


\section{CONTINUING MEDICAL EDUCATION (CME)}

\section{Current and Novel Approaches in DLBCL}

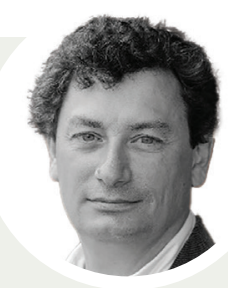

\section{FACULTY}

Gilles A. Salles, MD, PhD

University Professor

Head of Hematology

Claude Bernard University of Lyon

Hospices Civils de Lyon

Lyon, France

This activity was written by PER ${ }^{\circledR}$ editorial staff under faculty guidance and review. The Q\&A portion of the activity was transcribed from a recorded interview with the faculty and edited by faculty and $\mathrm{PER}^{\circledR}$ editorial staff for clarity.

\section{CME PROVIDER CONTACT}

\section{INFORMATION}

Physicians' Education Resource ${ }^{\circledR}$, LLC 2 Clarke Drive, Suite 110 Cranbury, NJ 08512 Toll-Free: 888-949-0045 Local: 609-378-3701

Fax: 609-257-0705 info@gotoper.com

\section{LEARNING OBJECTIVES}

Upon successful completion of this activity, you should be better prepared to:

- Explain current therapeutic regimens in newly diagnosed and relapsed/refractory DLBCL

- Evaluate efficacy and safety profiles of investigational treatment of DLBCL

- Describe mechanisms of action for novel targets to treat DLBCL

RELEASE DATE: September 1, 2020

EXPIRATION DATE: September 1, 2021

\section{INSTRUCTIONS FOR PARTICIPATION /} HOW TO RECEIVE CREDIT

\section{Read this activity in its entirety.}

2. Go to gotoper.com/go/dlbcl-journal20 to access and complete the posttest.

3. Answer the evaluation questions.

4. Request credit using the drop-down menu.

You may immediately download your certificate.

\section{FACULTY, STAFF, AND PLANNERS' DISCLOSURES}

In accordance with ACCME Guidelines, PER ${ }^{\circledast}$ has identified and resolved all COI for faculty, staff, and planners prior to the start of this activity by using a multistep process.

Disclosures (Dr. Gilles Salles): Consultant: AbbVie, Amgen, Autolus Therapeutics, Celgene, Gilead Sciences, Epizyme, Janssen Pharmaceutica, Kite Pharma, MorphoSys, Novartis, Roche, Servier Laboratories, Takeda Pharmaceutical Company.

The staff of $P E R^{\circledast}$ have no relevant financial relationships with commercial interests to disclose.

\section{OFF-LABEL DISCLOSURE AND DISCLAIMER}

This activity may or may not discuss investigational, unapproved, or off-label use of drugs. Learners are advised to consult prescribing information for any products discussed. The information provided in this activity is for accredited continuing education purposes only and is not meant to substitute for the independent clinical judgment of a healthcare professional relative to diagnostic, treatment, or management options for a specific patient's medical condition. The opinions expressed in the content are solely those of the individual faculty members, and do not reflect those of PER ${ }^{\circledR}$ or any of the companies that provided commercial support for this activity.

This activity is funded by PER ${ }^{\circledR}$.

\section{ACCREDITATION/CREDIT DESIGNATION}

Physicians' Education Resource ${ }^{\circledast}$, LLC, is accredited by the Accreditation Council for Continuing Medical Education (ACCME) to provide continuing medical education for physicians. Physicians' Education Resource ${ }^{\circledR}$, LLC, designates this enduring material for a maximum of 0.5 AMA PRA Category 1 Credits $^{T m}$. Physicians should claim only the credit commensurate with the extent of their participation in the activity. 
D iffuse large B-cell lymphoma (DLBCL) is a heterogeneous group of malignancies consisting of morphologically and clinicopathologically distinct subtypes and is the most common type of non-Hodgkin lymphoma (NHL). ${ }^{1}$ Standard therapy for the first-line treatment of DLBCL includes a regimen of rituximab, cyclophosphamide, doxorubicin, vincristine, and prednisone (R-CHOP). ${ }^{2}$ The complete course of R-CHOP for patients with advanced DLBCL consists of 6 cycles, with interim restaging after 2 to 4 cycles to confirm response. If the disease does not respond to R-CHOP or otherwise progresses, it is considered relapsed or refractory DLBCL, for which second-line systemic therapies are selected in consideration of patient- and disease-specific characteristics, such as whether the patient is a candidate to proceed to allogeneic hematopoietic cell transplant (HCT). Gilles A. Salles, MD, PhD, reviews recent data from clinical trials investigating emerging therapies for the treatment of DLBCL and how they will impact the treatment landscape and clinical management of patients with DLBCL in the near future.

\section{What is the evidence supporting the standard first-line treatment approach for patients with newly diagnosed DLBCL?}

SALLES: We first would like to request the prognostic parameters for patients with newly diagnosed DLBCL using the well-established international prognostic index (IPI). This index is composed of 5 parameters but can be easily simplified into an age-adjusted IPI, comprising only 3 parameters, which are Ann Arbor stage, LDH level, and Eastern Cooperative Oncology Group (ECOG) performance status. ${ }^{3}$ Further classifications are identified by the analysis of tumor tissues, such as the cell of origin, including germinal center B cell (GCB) versus non-GCB, also called activated $\mathrm{B}$ cell $(\mathrm{ABC}){ }^{4}$

Currently, rituximab combined with CHOP (R-CHOP) remains a gold-standard firstline therapy for most patients with DLBCL. ${ }^{2}$ There have been many attempts over the last 10 years to increase the value of R-CHOP, either by using a different anti-CD20 antibody, such as obinutuzumab, or adding maintenance after R-CHOP with rituximab, lenalidomide or other agents. What has been shown is that in most of the patients or all patients, 6 courses of R-CHOP are sufficient; however, patients with localized disease may do well with fewer courses of R-CHOP and may eventually achieve best molecular response established by some positron emission tomography, as the metabolic complete response may not need additional radiation therapy. ${ }^{2}$

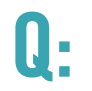

\section{How do you}

approach treatment

for patients with doublehit or double-expressor DLBCL?

SALLES: Patients with dou- ble-expressor DLBCL express the BCL2 and MYC antigens and have a worse outcome as compared with those without double expression. ${ }^{5}$ However, these patients are overrepresented in the non-GCB or $\mathrm{ABC}$ subtype. The standard of care is that these patients receive, like all other patients, R-CHOP therapy.

For patients with double-hit DLBCL, there is no established treatment option better than R-CHOP. ${ }^{2}$ Some physicians would like to use R-CHOP with a high risk of failure of recurrence. Others will use dose-adjusted R-EPOCH, which is a regimen that has shown great activity in patients with Burkitt lymphoma, and because Burkitt lymphoma has some molecular features close to this double hit, it has been envisioned to be helpful in these patients with double-hit DLBCL. ${ }^{6}$

\section{0.}

What is the treatment approach for patients with relapsed/ refractory DLBCL?

SALLES: For patients with relapsed/refractory DLBCL, we will first consider whether these patients are transplant eligible or not depending on age, comorbidities, and other considerations for getting access to this therapy. ${ }^{2}$ In older patients with advanced age, comorbidities, or those who do not respond to salvage therapy, transplant is not an option. And presently, the outcome of these patients is poor.

What are some of the key unmet needs and/or challenges in treating patients with relapsed/refractory DLBCL?

SALLES:There are several challenges in treating patients with relapsed/refractory DLBCL, one of which is that there are limited treatment options beyond transplantation. For patients who are not transplant eligible, the available treatment options may result in progression-free survival (PFS) of 3 to 6 months with currently available therapies and median overall survival (OS) is usually limited to 1 year. There is a great unmet need for nontransplant-eligible patients, as well as those patients who are on the path for transplant, but either failed salvage therapy or relapsed after transplant.

Q: How has CAR T-cell therapy changed the treatment paradigm for patients with relapsed/ refractory DLBCL?

SALLES: CAR T-cell therapy represents a new option for patients who have failed to respond to salvage therapy in the past for stem cell transplant, those who relapsed after stem cell transplant, or for a substantial proportion of patients not assessed as being transplant eligible. The results of the ZUMA-1 trial with axicabtagene ciloleucel, the JULIET trial with tisagenlecleucel, and the TRANSCEND trial with lisocabtagene maraleucel have established that the response rate in these difficult-to-treat patients can be achieved in a range of $50 \%$ to $80 \%$ with about $35 \%$ to $50 \%$ of patients achieving a 
complete response. ${ }^{7-9}$

Furthermore, results from phase 2 studies have shown that patients who were in complete response at 3 months after the infusion of CAR $\mathrm{T}$ cells had a probability of maintaining this response at 1 year or even 2 years or more, which was in the range of $70 \%$ to $80 \%$, leading to the hope that these patients may eventually be cured of their DLBCL. ${ }^{7,8}$ Based on these results, 2 of these products were already approved by health authorities in the United States and across the world, and a third one is also on its way. Recent data have also shown that results obtained in these phase 2 studies, which led to the approval of CAR-T therapies, were reproducible in the real-world setting. ${ }^{10}$

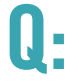

What are the key side effects associated with CAR T-cell therapy? How are these adverse events managed?

SALLES: The key adverse events associated with CAR T-cell therapy are cytokine release syndrome (CRS), neurological events, hematological toxicity, and recently, infectious complication, or more rarely, cardiac complications. ${ }^{7-10}$

Onset of CSR occurs within 7 days after CAR T-cell infusion and is characterized by fever, malaise, drop in blood pressure, and if not controlled at this time, may need additional support in an intensive care unit. Management of CSR includes treatment with an anti-IL-6 agent, such as tocilizumab, and steroids. CSR is reversible in the vast majority of cases, as long as the treatment is initiated early after the development of initial symptoms. $^{2}$

The second complication is neurotoxicity, specifically, immune effector cell-associated neurotoxicity syndrome (ICANS), and have a variety of symptoms that can range in severity, sometimes presenting as coma, seizure, or aphasia. ${ }^{2}$ In very few cases, cerebral edema was observed in patients, which can be fatal. This event occurs a little bit later, at 5 to 10 days after CAR T-cell infusion, and usually is preceded by CSR. Therapy involves high-dose steroids, dexamethasone, and antiepileptic drugs. Similarly, in the vast majority of patients, this side effect is reversible. Overall, we can say that CAR T-cell therapy is a relatively safe process.

\section{What are the potential barriers of CAR T-cell therapy for these patients? How might these challenges be overcome?}

SALLES: Despite the great enthusiasm regarding CAR T-cell therapy, there are barriers that prevent patient access to this treatment. First, CAR T cells need to be manufactured on an individual basis at the present time. ${ }^{11}$ In order to do that, patients will undergo leukapheresis. The leukapheresis product will be sent to the manufacturing site in the pharmaceutical company, and it will take usually 3 weeks to get back the product. If the patient has disease that is rapidly progressing, the patient may not be able to wait for the CAR T cells to be manufactured.

Additionally, CAR T-cell therapy needs to be performed in an experienced and qualified center, sometimes far away from where patients live. They have to travel and stay close to the center for the infusions. This can be difficult for some patients. Lastly, CAR T-cell therapy at the present time is expensive, and patients should be covered by their insurance in order to access to this new treatment.

In the future, there are different ways of seeing this CAR T-cell therapy becoming available for a larger proportion of patients by diminishing the toxicities, having more rapid manufacturing times, by combining CAR T cells with other agents, which may improve efficacy in those patients who don't get the greater benefit of CAR T cells. Another path which is currently under exploration is to use allogeneic CAR T cells, which are manufactured from the leukocytes of a healthy donor and then are immediately available for treatment. However, we don't know at this present time whether these CAR T cells will persist and achieve cure.

\section{- How do you decide on treatment strategies for patients with relapsed/refractory DLBCL who are ineligible for transplant and CAR T-cell therapy?}

SALLES: There are, unfortunately, few treatment strategies available for patients who are ineligible for transplant or CAR T-cell therapy.
Conventional chemotherapy, including those based on cytarabine, bendamustine, and other combinations, provide unsatisfactory results with response rates in the range of $40 \%$ to $50 \%$, with about $20 \%$ of patients achieving complete responses that are not durable in most patients. ${ }^{2}$ We clearly need new agents for these patients who are nontransplant eligible in the second or third line of therapy.

\section{What are the key $\checkmark$ - clinical data with selinexor reported in the SADAL trial? How will the approval of this agent change treatment in the relapsed/refractory DLBCL setting?}

SALLES: Selinexor is a new compound targeting the intranuclear exporter. The drug was assessed in patients with very advanced disease. The overall response rate that was achieved was $28 \%$, with $12 \%$ of patients achieving a complete response. ${ }^{12}$ The response rate did not differ widely for patients with a GCB subtype or a non-GCB subtype. The median PFS was 2.6 months and the median OS was 0.9 months. It is a new agent of a new therapeutic class, which is providing response in a small number of patients, but some of these responses were durable.

\section{What are some of agents under investigation for the treatment of relapsed/refractory DLBCL?}

SALLES: Many new agents 
have been investigated in patients with relapsed/refractory DLBCL. For patients with non-GCB subtype, drugs such as CDK inhibitors have shown modest efficacy, with response rates of about $20 \%$ to $30 \%$ that are not very durable. A drug that has recently been approved in combination with a chemotherapy backbone composed of bendamustine and rituximab was polatuzumab vedotin. ${ }^{13}$ This antibody-drug conjugate, when combined with these agents, provided a complete response rate of $40 \%$ in patients who are nontransplant eligible and the median PFS for these patients was close to 1 year, which was a clear benefit as compared with bendamustine and rituximab alone or with other combinations that are used in these patients. This led to the approval of the combination of polatuzumab vedotin with bendamustine/ rituximab in the third-line setting in the United States and in the second- and third-line settings elsewhere in Europe.

Another investigational agent, tafasitamab, targets CD19. As a single agent, it has a modest efficacy in B-cell NHL. However, results from a recent study called L-MIND have shown that the combination of tafasitamab with lenalidomide in patients with relapsed DLBCL led to a $60 \%$ response rate, with $40 \%$ of the patients achieving a complete response with a median duration exceeding 18 months. ${ }^{14} \mathrm{~A}$ recent study called RE-MIND was designed to compare the results of the tafasitamab/ lenalidomide combination from the L-MIND study with the real-world evidence of the activity of lenalidomide alone, and the results showed that response rate, PFS, and OS were greatly increased by the combination of tafasitamab plus lenalidomide; hopefully, this combination may be available to patients in the near future. ${ }^{15}$ Another strategy currently under exploration involves antibodies directed against specific antigens, such as $\mathrm{CD} 19, \mathrm{CD} 79, \mathrm{CD} 22$, which are antibodies coupled with a toxin. ${ }^{16}$ Coltuximab ravtansine is one of these agents with promising results, and other compounds in this family are being actively developed. ${ }^{17}$

\section{What is the rationale for bispecific antibodies and how do they work?}

SALLES: Bispecific antibodies are designed to bind to tumor cells by recognizing an antigen, usually CD20 or potentially CD19, and to bind to the T cell. ${ }^{18}$ By doing so, the $\mathrm{T}$ cell comes in close contact with the tumor cell and is activated, subsequently killing the tumor cell expressing the antigen.

One of the first bispecific antibodies developed is blinatumomab, which was developed first for acute lymphoblastic leukemia and has shown promising results in patients with aggressive lymphoma. ${ }^{19}$ Treatment with this drug is limited by its neurological side effects and the need for continuous infusion of the drug over 1 week or more.

Several other antibodies designed to target both $\mathrm{CD} 3$ and CD20 are undergoing investigation. ${ }^{20}$ Some responses from early studies were durable. Adverse events included infusion-related reactions. Few patients presented with neurological toxicity. Overall, this appears to be a promising family of drugs.

What immune
checkpoint
inhibitors are in
development for relapsed/
refractory DLBCL? Which
patients would benefit
from this treatment
approach?

SALLES: Overall, treatment of relapsed/refractory DLBCL using the anti-PD-1 or antiPD-L1 antibodies has had a limited response in trials. Given the weak efficacy with these agents, other immune checkpoint blockers are being investigated. Recently, it was discovered that tumor cells may defend themselves by expressing the CD47 antigen, which provides an inhibitory signal to macrophages. ${ }^{21}$ Therapeutic strategies were attempted, leading to the development of investigational anti-CD47 antibodies. The mechanism results in macrophages engulfing and killing tumor cells; there is thought to be a synergistic effect when combined with other antibodies, such as rituximab, which is currently being explored. Early results demonstrated a favorable effect, which must be confirmed in future studies.

Recent updates to the NCCN guidelines for B-cell lymphomas include tafasitamab plus lenalidomide as a category $2 \mathrm{~A}$ recommended therapy for the second- and subsequent-line treatment of patients with DLBCL who are not candidates for transplant. $^{2}$

\section{KEY REFERENCES}

2. NCCN. Clinical Practice Guidelines in Oncology. B-cell lymphomas, version 4.2020. Accessed August 14, 2020. https://www.nccn.org/ professionals/physician_gls/pdf/bcell.pdf

12. Kalakonda N, Maerevoet M, Cavallo F, et al. Selinexor in patients with relapsed or refractory diffuse large B-cell lymphoma (SADAL): a singlearm, multinational, multicentre, open-label, phase 2 trial. Lancet Haematol. 2020;7(7):e511-e522. doi:10.1016/S23523026(20)30120-4

14. Salles G, Duell J, González Barca E, et al. Tafasitamab plus lenalidomide in relapsed or refractory diffuse large B-cell lymphoma (L-MIND): a multicentre, prospective, singlearm, phase 2 study. Lancet Oncol. 2020;21(7):978-988. doi:10.1016/ S1470-2045(20)30225-4

17. Hicks SW, Tarantelli $C$, Wilhem A, et al. The novel CD19targeting antibody-drug conjugate huB4-DGN462 shows improved anti-tumor activity compared to SAR3419 in CD19-positive lymphoma and leukemia models. Haematologica. 2019;104(8):16331639. doi:10.3324/ haematol.2018.211011 journal20 


\section{Urology Times}

Leading research and analysis.

Practical advice.

\section{www.UrologyTimes.com}




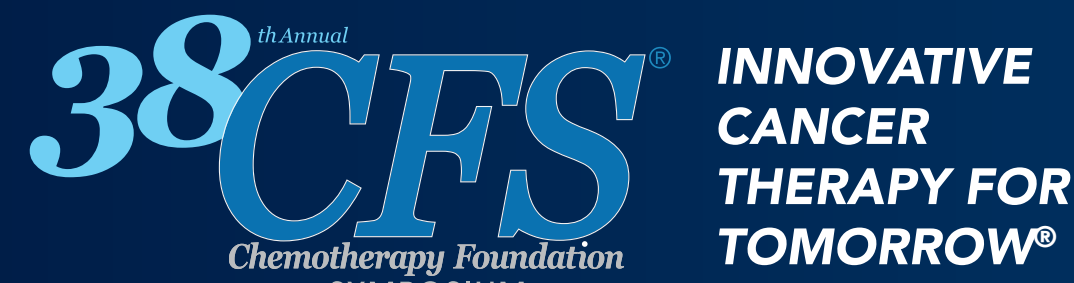

SYMPOSIUM

VIRTUAL, INTERACTIVE CONFERENCE

NOVEMBER 4-6, 2020

\section{Turning today's cancer therapy breakthroughs into tomorrow's patient care strategies.}

\section{BENEFITS OF ATTENDING}

- Turn breaking data on cancer therapy into actionable strategies that optimize outcomes for your patients across 25 tumor types

- Get answers to your top treatment challenges and network with 100+ internationally renowned cancer care experts

- Participate in discussions and Q\&A sessions using our custom, interactive platform

- Gain evidence-based cancer management strategies that you can apply immediately in your patient care

\section{PROGRAM CHAIRS}

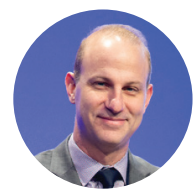

Benjamin P. Levy, MD Johns Hopkins Sidney Kimmel Cancer Center Washington, DC

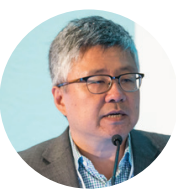

William K. Oh, MD

Tisch Cancer Institute New York, NY

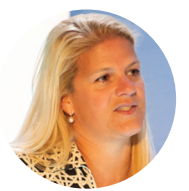

Tiffany A. Traina, MD Weill Cornell Medicine New York, NY

\section{REGISTRATION FEES}

Physicians

Fellows

Nurses, PAs, other HCPs Industry

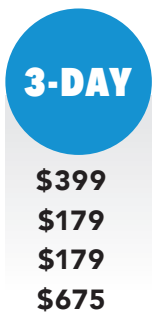

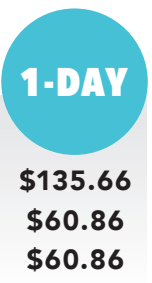

To attend for one day only, use code CFS20Daily at checkout*

Learn more at gotoper.com/go/CFS20ONC

*Discount applies to health care professionals only. Other restrictions may apply

Accreditation/Credit Designation

This activity has been planned and implemented in accordance with the accreditation requirements and policies of the Accreditation Council for Continuing Medical Education (ACCME) through the joint providership of Physicians' Education Resource ${ }^{\oplus}$, LLC, and Pharmacy Times Continuing Education. Physicians' Education Resource ${ }^{\oplus}$, LLC, is accredited by the ACCME to provide continuing medical education for physicians.

Physicians' Education Resource ${ }^{\oplus}$, LLC, designates this live activity for a maximum of 24.25 AMA PRA Category 1 Credits ${ }^{\text {TM }}$. Physicians should claim only the credit commensurate with the extent of their participation in the activity.

Pharmacy Times Continuing Education ${ }^{\mathrm{TM}}$ is accredited by the Accreditation Council for Pharmacy Education as a provider of continuing pharmacy education.

AC This activity is approved for 21.75 contact hours (2.175 CEUs) under the ACPE universal activity numbers:

PE. 0290-9999-19-196-L01-P; 0290-9999-19-197-L01-P; 0290-9999-19-198-L01-P

Physicians' Education Resource ${ }^{\oplus}$, LLC, is approved by the California Board of Registered Nursing, Provider \#16669, for 24.25 Contact Hours.

Acknowledgment of Commercial Support

This activity is supported by educational grants from AstraZeneca; Boehringer Ingelheim Pharmaceuticals, Inc.; Bristol Myers Squibb; GlaxoSmithKline; Ipsen Biopharmaceuticals, Inc; Janssen Scientific Affairs, LLC and Pharmacyclics LLC, an AbbVie Company; Taiho Oncology, Inc; Novartis Pharmaceuticals Corporation; Seattle Genetics, Inc.; and Jazz Pharmaceuticals, Inc. 